DOE/ID/12018-1

\title{
MASTER Colorado Geothermal Commercialization Program
}

\author{
Semi-Annual Report \\ January - June 1979
}

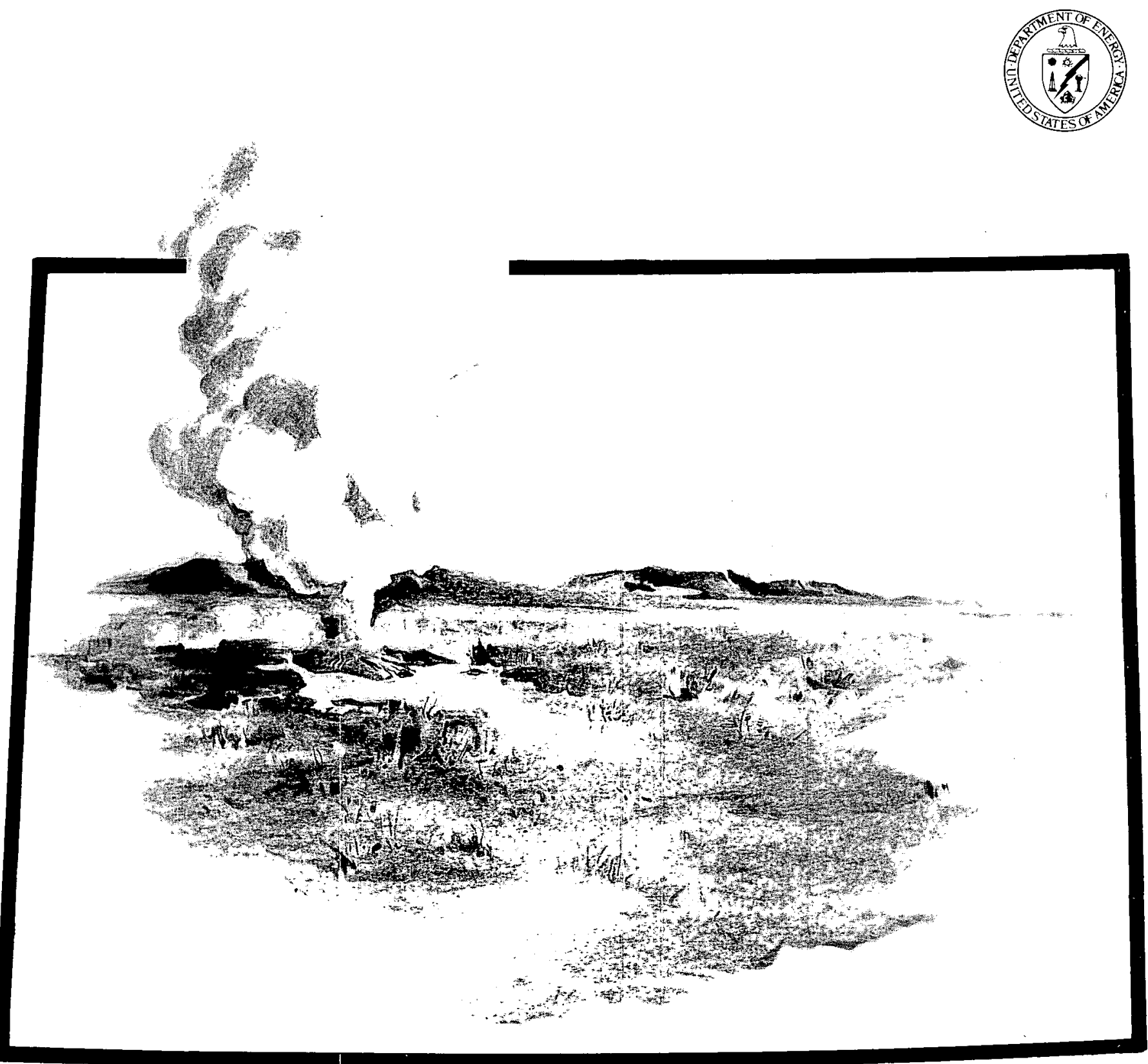




\section{DISCLAIMER}

This report was prepared as an account of work sponsored by an agency of the United States Government. Neither the United States Government nor any agency Thereof, nor any of their employees, makes any warranty, express or implied, or assumes any legal liability or responsibility for the accuracy, completeness, or usefulness of any information, apparatus, product, or process disclosed, or represents that its use would not infringe privately owned rights. Reference herein to any specific commercial product, process, or service by trade name, trademark, manufacturer, or otherwise does not necessarily constitute or imply its endorsement, recommendation, or favoring by the United States Government or any agency thereof. The views and opinions of authors expressed herein do not necessarily state or reflect those of the United States Government or any agency thereof. 


\section{DISCLAIMER}

Portions of this document may be illegible in electronic image products. Images are produced from the best available original document. 


\section{NOTICE}

Reference to a company or product name does not imply approval or recommendation of the product by the Colorado Geological Survey or the U.S. Department of Energy to the exclusion of others that may be suitable.

This report was prepared as an account of work sponsored by an agency of the United States Government. Neither the United States nor any agency thereof, nor any of their employees, makes any warranty, expressed or implied, or assumes any legal liability or responsibility for any third party's use or the results of such use of any information, apparatus, product or process disclosed in this report, or represents that its use by such third party would not infringe privately owned rights. 


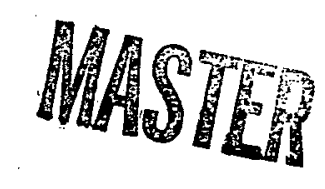

DOE/ID/12018-1

\title{
COLORADO GEOTHERMAL COMMERCIALIZATION PLANNING
}

\author{
SEMI-ANNUAL PROGRESS REPORT \\ January 1, 1979 - June 30, 1979
}

Prepared by:

Barbara A. Coe

\author{
Colorado Geological Survey \\ Department of Natural Resources \\ Denver, Colorado 80203
}

Work performed under Contract No. DE-FC07-79ID12018

U.S. Department of Energy

Idaho Operations Office

DISCLAIMER

This book was prepared as an account of work sponsored by an agency of the United States Government. Neither the United States Government nor any agency thereof, nor any of their employees, makes any warraniy, express or impled, of assumes any legal lizbility or responsibility for the accuracy,
completeness, or usefulness of any information, doparatus, product, or isocess disclosed, or represents that its use would not inftinge privately owned rights. Reference herein to any specific commercial product, process, or service by trade name, trademark, manufactuser, or otherwise, does States Government or any agency thereof. The views and opinions of authors expressed herein do not necessarily state or reflect those of the United States Government or any agency therect. 


\section{Acknowledgements}

The persons who provided information and assistance leading to this compilation are numerous. Sincere appreciation is extended to all those who participated. A special thank you to Richard H. Pearl, for his freely contributing from his store of knowledge of geothermal resources in Colorado; to Dr. Richard T. Meyer, for his most capable leadership and direction, to Nancy Forman for drafting the institutional section, and to Valerie Taylor and Becky Andrews for their untiring manuscript typing, and to my husband, David, for his willing and capable review. 
TABLE OF CONTENTS

$\underline{\text { SECTION }}$

PAGE

1.0 Introduction

1.1 Purpose of Project

1.2 Objectives

1.3 Technical Approach and Team Members

1.4 Benefits of Project to State and DOE

2.0 Summary of State Project Tasks and Work Accomplished 5

Task 1: Applications Analysis

Task 2: Utilization Analysis and Prioritization

Task 3: Site-Specific Development Plans

Task 4: Selected Time-Phase (Heber-Type) Commercialization Project Plan

Task 5: State Handbook of Institutional Procedures

Task 6: State and Site Energy and Economic Analyses

Task 7: State and Regional Aggregations of Development Plans

Task 8: Identification of Constraints and Recommended Actions

Task 9: State Outreach Program

3.0 Specific Task Descriptions and Products 12

3.1 Geothermal Prospect Identification $\quad 12$

3.2 Area Development Plans

3.2.1 State Geothermal Planning Areas

3.2.2 Area Development Plan

3.3 Site Specific Development Plans 33

3.3.1 Candidate Geothermal Sites/Applications 33

3.3.2 Site Specific Development Plans in Preparation 33

3.3.2.2 Sites with Development Activity 34

3.4 Time Phased Project Plans 37

3.4.1 Active Demonstration/Commercialization Projects 37

3.4.2 Time Phased Project Plans--Completed 37

3.5 State Aggregates of Prospective Geothermal Utilization 38

3.6 Institutional Analysis 40

3.6 .1 Overview of State Laws and Regulations $\quad 40$

3.6.2 Institutional Procedures 46

, $0 i 1$ and Gas Conservation Commission 47

$\begin{array}{ll}\text { State Engineer } & 49\end{array}$

State Board of Land Commissioners 50

Water Quality Control Commission 51

Public Utilities Commission

Air Pollution Control Commission $\quad 52$

State Land Use Commission 53

State Highway Department.

State Tax Incentives $\quad 54$

3.7 Public Outreach Program $\quad 64$

3.7.1 Outreach Mechanisms $\quad 64$

3.7.1.1 Existing

3.7.1.1.1 Media Publicity 
3.7.1.1.2 Meetings and Telephone Interviews

3.7.1.2.1 Industry and Agribusiness Contacts

3.7.1.1.2.2. Interagency Coordination

3.7.1.1.2.3 Communities and User Contacts

3.7 .1 .1 .3

Lectures

3.7 .1 .1 .4 Workshops

3.7.1.1.5 Displays, Brochures, Papers

3.7.2 Summary Listing of Contacts and Results

3.7.1.2. Recommended Mechanisms

4.0 Summary of Major Findings and Recommendations 76

4.0.1 Electrical Power Potential 76

4.0.2 Direct Thermal Potential $\quad \therefore \quad 77$

4.0.3 Recommended Actions 84

*Appendix A Geothermal Energy Development Potential in Planning Region 8 San Luis Valley, Colorado

*Appendix B A Preliminary Scenario for Development of Geothermal Resources in Colorado

*Appendix C Geothermal Energy Potential in Pagosa Springs, Colorado

*The appendices have not been printed as part of this report. Information pertaining to the appendices can be obtained from the Colorado Geological Survey, Department of Natural Resources, 715 State Centennial Building, 1313 Sherman Street, Denver, Colorado 80203 (Attn: Barbara Coe). 
TABLE

PAGE

1 Colorado Hydrothermal Sites $\quad 15$

2 Estimated Reservoir Temperatures 16

3 Resource Assessment 23

4 Federal Active Competitive Geothermal Leases 25

5 Federal Active Non-Competitive Geothermal Leases 26

6 Colorado State Active Geothermal Lease $\quad 27$

7 Current Uses of Geothermal Resources 28

8 Some Potential Uses for Geothermal Resources 30

9 State of Colorado Annual Employment Data 78

FIGURE

1 Location of Thermal Springs and Wells in Colorado 13

2 Location of Colorado Region 8

3 Procedures for Obtaining Geothermal Permits--0il and Gas Conservation Commission $\quad 57$

4 Procedures for Obtaining Geothermal Permits--State Engineer 59

5 Procedures for Obtaining Geothermal Leases--State Board of Land Commissioners

6 Procedures for Obtaining Discharge Permits 


\subsection{Introduction}

\subsection{Purpose of Project}

The State of Colorado seems to have significant geothermal energy potential. Indeed, several successful geothermal facilities are located in the state already. A prior study showed that about 500 megawatts of electricity could be produced in Colorado with geothermal energy and that about 30 communities could use geothermal for their direct thermal energy needs (Coe, 1978).

A more specific indication of the potential is necessary, however, for several reasons. First, tax dollars are allocated by the Federal government to aid the research and development of various energy forms, including geothermal energy. The extent to which the expenditure of public funds is justified depends upon the extent of the probable return of benefits to the public. In the case of geothermal energy, these benefits come in the form of energy on 1 ine at an affordable price and without intolerable negative impacts.

Secondly, the Congressional mandate that led to this planning effort requires the encouragement of the commercial development of geothermal energy (P.L. 93-410, Sec. 2). It is hoped that providing ideas and information about the geothermal energy potential may help stimulate local communities, users, and developers to develop and use a valuable, but so-far virtually-untapped resource. As such, the Colorado Geological Survey, under a contract from Four Corners and the Department of Energy, is evaluating the potential for developing the geothermal resources of Colorado. This report details that potential. Finally, the study describes constraints that are limiting geothermal energy development. Recommendations may then be made for measures designed to overcome those constraints.

\subsection{Objectives}

To attain the goals described, the Colorado Team established several related objectives. First, an analysis of one broad but homogeneous area would define the market potential of the energy in that area, while at the same time establishing a procedure and information requirements for subsequent analyses. The San Luis Valley Region was chosen for this analysis because of its relative 
homogeneity and the availability of necessary information.

Secondly, a detailed time-phased analysis of one firm geothermal prospect was stipulated in order to learn the detailed steps required for development, to the extent possible, based upon experience. This information will be used to help postulate subsequent developments, as well as to help guide prospective developers.

The third group of analyses, site-specific ones, are designed to show the proposed and potential applications at sites throughout the state. Newly-obtained information will allow the refinement and expansion of those site-specific scenarios prepared last year so that they will more realistically reflect the potential for geothermal development.

An applications analysis was directed toward identification of the current and potential uses of geothermal energy in the state. The potential applications in each area are based upon the current and forecast. industries, as well as residential and commercial needs. Site energy and economic analyses are being conducted as part of each area or site analysis. In addition, statewide data have been analyzed in order to identify those areas that offer the highest potential for geothermal energy use. Whereas activity in areas with declining economies could be stimulated if geothermal energy were developed, geothermal energy development could help ease the socio-economic and environmental impacts of development in areas of high growth and economic activity.

$\dot{A}$ State Handbook of Institutional procedures is intended to be a guide for participants or prospective participants in geothermal energy development. The basis for development of that handbook is the institutional analysis in this report.

An aggregation of postulated energy on line for each of the areas and sites in Colorado is designed to show the total potential for geothermal energy use throughout Colorado. Institutional, technological, environmental, and other constraints to that development will help to identify and recommend solutions to those problems.

Finally, the last task for which Colorado contracted, an outreach program, is designed to help overcome one type of constraint to geothermal development, the 
lack of knowledge. Information about the energy, its applications, procedures, and techniques for development is widely distributed to appropriate groups to fill this need.

\subsection{Technical Approach and Team Members}

The overall approach to accomplishing the indicated objectives is a "grass roots" approach. Just as the Department of Energy in Washington, D.C. cannot know much about the activities, needs, and goals of geothermal participants in each state except through a central contact who maintains a statewide communication network, the state geothermal team cannot know much about the activities, needs, and goals at the geothermal areas themselves without communicating with the people who are there. Usually, no published information is available concerning geothermal development, nor for that matter, current or possible related development. Too, an idea is only implementable to the extent that it is desired and pushed by those who must implement it. The approach of the Colorado Team is, therefore, to initiate a dialogue with residents, developers, and key officials in each area to be investigated and to maintain that dialogue not only through the analysis, but it is hoped, through a subsequent development phase as well.

The analyses, however, rely heavily upon published sources for required background information, to avoid re-creation of work previously done. Published information includes information about population, energy demand, geographical and economic characteristics, requirements for and special conditions relative to geothermal development in a given area, estimated energy available, and the resource area characteristics.

Quantitative analyses are performed when necessary to forecast or estimate energy demands, population, energy availability, or other elements.

Based upon this information, with the collaboration of the site advisory groups, possible development is postulated, along with a development schedule. Details of the utilization and development analysis methodology are described in detail in each of those analyses.

Team members for the project are Richard H. Pear1, Chief, Groundwater 
Investigations Section, administrator of the project; Barbara Coe, Project Chief. Periodically, temporary research assistants are employed to assist with particular investigations and analyses.

a

1.4 Benefits of Project to State and DOE

The total benefits of this project are impossible to quantify, except for the $\$ 57,000.00$ project budget that is spent almost entirely within the state. However, the economic benefits could be far-reaching. Some possible ones are:

1. Reduction in energy costs for state residents.

2. Increase in energy availability for industry, commerce and residents.

3. Increase in incomes and employment rates in depressed areas that have geothermal resources.

4. Increased stabilization of infrastructure and costs in high-growth areas that have geothermal resources.

For the U.S. Department of Energy (DOE), the benefits include the acquisition of accurate, up-to-date information about geothermal development activities and an assessment that is based on detailed information of the geothermal market potential. Furthermore, the outreach program helps spread the awareness of geothermal energy and encourage its development, enhancing the DOE geothermal program.

For the country, it is hoped that this unused energy will be employed, benefitting the nationwide economy by enhancing the production opportunities either directly or indirectly by releasing other fuels, and by reducing the imports of energy fuels. 


\subsection{Summary of State Project Tasks and Work Accomplished}

The Colorado Geological Survey contracted to do the tasks 1 isted below as part of the Regional Geothermal Project. The tasks and their results are summarized as follows:

Task 1: Applications Analysis

This task required a survey and analysis of current and potential applications/uses of geothermal energy, including both electric and direct thermal applications, conducted by examining historical and projected patterns of energy consumption by industry, agriculture, commerce, private residences, and local government units. Information is derived from reports of the Public Utility Commission, the State Energy Conservation Office, U.S. Bureau of Census reports, and DOE documents, utility companies and users. New applications for geothermal energy, such as industrial and agricultural processing, are and will continue to be identified, described, and examined with respect to future energy requirements.

For a number of areas and sites in the state, estimated current and projected energy demands to the year 2020 that could be supplied by geothermal energy were compiled. Commercial, industrial, and residential sectors are specified. The remaining applicable areas of the State will be analyzed subsequently. Not only is this information vital to understanding the potential for geothermal energy, but it is also an indicator of the constraints and possible incentives for that development. Further, it helps provide a basis for prioritization of areas for concentration.

Geothermal energy could make a substantial contribution to supplying the energy demands of the tourism and energy development industries. These are two of the largest industries in Colorado, particularly in those areas of colorado where geothermal resources have been identified. In addition, some industrial demand and agricultural processing potential for geothermal is indicated in some areas, such as those that have high timber and field crop production. 
Task two was a complete utilization evaluation of one planning region consisting of five to six counties for which detailed information regarding energy requirements has been completed. This evaluation was to be directed at the industrial and commercialization applications of geothermal energy in addition to space heating and to show how the resource could be developed between the present and the year 2020 .

Such an analysis was prepared for the San Luis Valley Region of south-central Colorado. It describes the current geothermal energy on line, including heat for swine houses and fish culture, the planned facilities including additional swine houses and proposed facilities such as a barley-malting plant and greenhouses. The results showed that nearly all the thermal energy demand in the San Luis Valley communities to the year 2020 could be supplied by geothermal energy. Discussions with State and San Luis Valley officials of the analysis have identified some of the constraints to development and some possible solutions to those problems. Furthermore, selection of the prime areas for concentrating efforts to help encourage development relied upon the analysis.

Task 3: Site-Specific Development Plans

This task stipulated the preparation of plans for each major geothermal prospect that has development potential between now and the year 2020. They would originate with the utilization analysis and prioritization of Task 2 , and yield the basic information upon which selected time-phase (Heber-type) commercialization project plans for Task 4 could be evolved. Semiquantitative examination of technological, economic, environmental andinstitutional factors which influence prospect development were indicated in order to provide a rea1-world, real-time character to the site-specific development plans. Direct contact would be established with the active or potential resource developers and with the prospective industrial, commercial, agricultural, and governmental users, to ascertain the magnitude and timing of their actions. The actions required by government agencies (local, state, and federal) to achieve or to accelerate the magnitude and rate of the geothermal development and utilization would be identified and the several site-specific development plans would be 
sufficiently uniform in content and complete in detail so that state and regional aggregations could be carried out in collaboration with the New Mexico Energy Institute (NMEI) (Task 9).

The information required for site-specific plans has been accumulated for the sites that will be analyzed during this contract year. Those sites include the Town of Ouray, a town that has a geothermally-heated municipal garage, has been actively working to develop their geothermal energy and has relied upon high-cost propane and electricity for its heat. Idaho Springs, a small town near Denver, another site for which a plan is being prepared, is near a major metropolitan area and has within it a hydrotherapy resort whose owners are actively trying to develop a geothermal heating system for the building. Town administrators are interested in the district heating potential.

The third area to be analyzed is Glenwood Springs, a town with several springs, one of which flows $2,263 \mathrm{gpm}$ at $50^{\circ} \mathrm{C}$ (Barrett and Pearl, 1978). Here, too, community members are attempting to initiate district heating. The last area is the Animas Valley, an area that includes an industrial park as well as housing and commercial and public buildings with an immediate prospect of heating a private boarding school.

These analyses identify the potential for geothermal energy and through the "grass roots" process of preparation, help to increase awareness of that potential. Following completion of each of the analyses, outreach efforts will focus on encouraging expansion of development efforts.

Task 4 - Selected Time-Phase (Heber-Type) Commercialization Project Plan

Preparation of one time-phase commercialization plan for a selected site and application which is in the advanced stages of development or commercialization by industry, agriculture, commerce, or government was indicated. The site and associated application selection, made as a joint decision of the state team leader, the EG\&G technical project manager, and the DOE geothermal program office, was the Pagosa springs district heating system being developed with the aid of DOE funds.

The time-phase commercialization plan follows the technical approach, format, 
and content of the plan developed by Science Applications, Inc., for the Heber geothermal prospect and electrical application (cross-reference "Heber Electric Scenario," Science Applications, Inc., March 3, 1978). Preparation of the plans required close communication with the city, the contract engineer, and governmental agencies in order to specify the step-by-step procedures, the actions necessary, and the times required to achieve full-scale commercialization. Detailed consideration was given to the technical, environmental, economic, market, institutional, legal, and political factors which affect and/or control the success of commercialization in a timely manner. The state team utilized the findings of Task 5 on institutional analysis as the basis for certain aspects of Task 4 and in turn provided input to Task 5 from analysis of the institutional requirements for Pagosa springs development. This, analysis shows the central district heating about 60 buildings by winter of 1980, with a strong possibility of heating the remainder of the town by 1983 and suburban areas at a later time.

The analysis provides information for subsequent analyses, as well as helping to clarify and smooth the scheduling of the activities required by Pagosa Springs.

Task 5: State Handbook of Institutional Procedures

$\dot{A}$ state handbook of the governmental regulations, procedures, and practices that were identified and charted during 1978 will be prepared. The handbook is intended to be an information manual and a procedural guide for a full array of parties who may participate in geothermal development and commercialization, including, but not limited to, investors, financial institutions, developers, utilities, energy users, industry, commerce, business, and local governments. The handbook will chart and describe the agencies, regulatory procedures, action or decision times, and procedural costs in a clear and easily readable form. The specific format for the handbook prescribed by DOE/EG\&G will be followed.

Information for the handbook has been collected and is being compiled. The handbook will provide in published form an easy-to-follow guide to the governmental requirements for geothermal development in Colorado. The guide is designed to 1 . satisfy numerous requests for information as well as to 2 . provide information that might simplify the development process and make it 
more efficient, 3. remove the psychological barrier created by the uncertainty about the process, to help. stimulate or at least reduce the barriers to geothermal development.

\section{Task 6: State and Site Energy and Economic Analyses}

Energy and economic data are collected and analyzed to support other tasks and to assist NMEI in their economic analyses and energy forecasting activities.

Data collected include energy consumption, energy cost, population, income, manufacturing, and others to provide the basis for CGS analyses and also for NMEI analyses. In addition to indicating the nature and magnitude of the market for geothermal energy for planning purposes, these data provide a concrete illustration to prospective developers of the market potential of geothermal energy.

Task 7: State and Regional Aggregations of Development Plans

This task requires state and regional aggregations of the utilization prospects from Task 2 and the site-specific development plans from Task 3 in collaboration with NMEI. The purpose of the aggregations is to obtain estimates of the amount of geothermal energy that can be commercialized between now and the year 2020. The state and regional aggregations are carried out separately for the following three geothermal prospect classifications: proven reserves, potential resource, and inferred resource.

In Colorado, because of the dearth of exploration activity in Colorado, all sites are classified as inferred except for Pagosa springs which is a "potential" site. Both electric and direct thermal applications are evaluated within the aggregations of the geothermal prospect classifications: Colorado concentrates on the technological, environmental, and institutional factors in providing input to the aggregations, whereas NMEI concentrates on the economic and energy/demand factors in finalizing the aggregations. NMEI uses computerized aggregation procedures, with. Colorado providing complete and consistent data to the extent it is available. NMEI makes available the draft and final state and regional aggregations to the state team for review prior to publication and for use in the state outreach program. 
Aggregations for estimated residential energy demand of all Colorado sites were prepared during the last contract period. These are being revised, and expanded to reflect detailed energy demand and other new data. These aggregations will reveal the estimated total potential of geothermal energy for direct use in resource areas of Colorado. Prospective developers have almost unanimously indicated need for grant funds to help bear the cost of such development. For electrical power sites, leases, in several cases, on National Forest and in one case, on private land, are needed. While nothing can assure the issuance of leases on private land, clarification and coordination of Federal policies among. Federal agencies could alleviate conflicts. Showing that the energy potential of geothermal resources is sufficiently large and widespread can enhance the interest in its development.

Task 8: Identification of Constraints and Recommended Actions

The products of the above tasks are examined in order to identify the various technological, environmental, economic, and institutional constraints which might delay or prevent the development and commercialization of geothermal energy in Colorado and in the region. Both generic and specific constraints are identified. Possible solutions to the problems are suggested and evaluated, and actions necessary to both the public and private sectors to remedy the problems or implement the solutions are recommended.

These constraints and recommendations are documented in detail in Section 4.0 of this report. The lack of front-end financing, lack of skilled and knowledgeable experts to perform the technical tasks necessary for resource development and lack of motivation are three primary constraints to geothermal development.

Task 9: State Outreach Program

The State outreach program is organized and conducted in Colorado for the purpose of promoting the utilization of geothermal energy by industry, utilities, commerce, agriculture, business, and governmental units. Colorado draws upon the activities of the other tasks and upon the resources of NMEI, $E G \& G$, and DOE to carry out their outreach program. In particular, the state team is required to provide information on geothermal prospects, applications, 
economics, energy supply/demand projections, institutional procedures, and environmental advantages to various parties to keep a record of its outreach contacts and activities.

The Colorado Team has contacted numerous industry, educational, community, State and Federal officials and individuals to supply information and assistance to help stimulate the development of geothermal energy.

During the past year, at least five of the parties contacted submitted Federal grant proposals for either more detailed study or development projects. About 10 of those contacted requested technical assistance, feasibility study assistance, or additional technical data. Either such assistance or a referral to EG\&G were provided. At every resource area visited, interest in geothermal energy was high and at most, some uses or attempts to use the energy were obvious. Lack of capital for development was an almost universal problem. 


\subsection{Specific Task. Descriptions and Products}

Each of the geothermal development analyses compiled for the State of Colorado, both during this period and previously as part of this project, is included in this section. Also included is a description of each of the geothermal prospects, of the institutional procedures required for geothermal development in Colorado, and of the outreach program that is designed to help encourage the development of geothermal energy in the state.

\subsection{Geothermal Prospect Identification}

The identified and verified thermal springs and wells in the state are shown on the map, Figure 1. n a recent inventory of thermal wells and springs in the state, temperatures and chemical content were described (Barrett and Pearl, 1978). Table 1 shows the areas, their locations and measured surface temperatures. Subsequently, subsurface temperatures, reservoir volumes, and energy content were estimated (Pearl, in prep.). These data are shown on Tables 2 and 3 . Several additional areas have become known since the inventory was conducted and still others have been reported to CGS personnel, but are not yet verified. The full extent of the hydrothermal resource may be much vaster than is apparent from existing surface expressions.

Several of these areas in Colorado have been leased by energy developers. Although information about leases on private land is not available, leases on public lands are 1 isted on Tables 4, 5, and 6. As shown, 3 leases are active on federal KGRA's, 22 leases are active on other federal 1 ands and 16 leases are active on State lands. Current users are shown on Table 7. The general list of possible applications of geothermal energy derived from published sources, government agencies, and industry representatives on Table 8 shows additional uses. However, until a geothermal resource site is identified that offers conditions conducive to a given application, that use cannot be considered a potential.

\subsection{Area Development Plans}

Because the analysis of the geothermal development potential in Colorado that was conducted last year resulted in site specific scenarios (Coe, 1978), the 


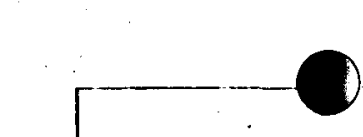

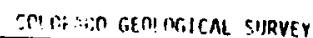
WYOMING

NEBRASKA $a A$

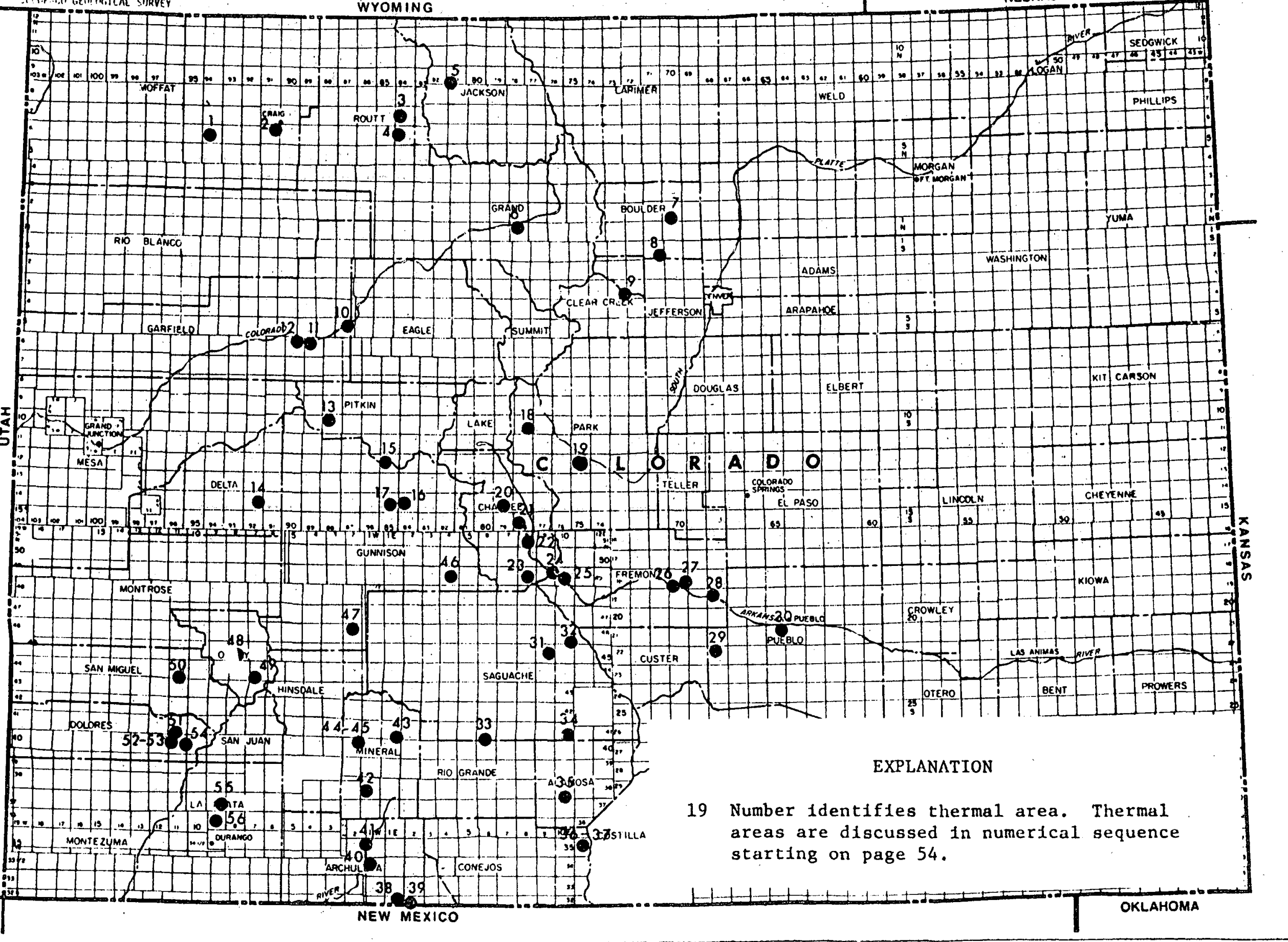

Figure 1. Location of thermal springs and wells in Colorado. 


\section{COLORADO HYDROTHERMAL SITES}

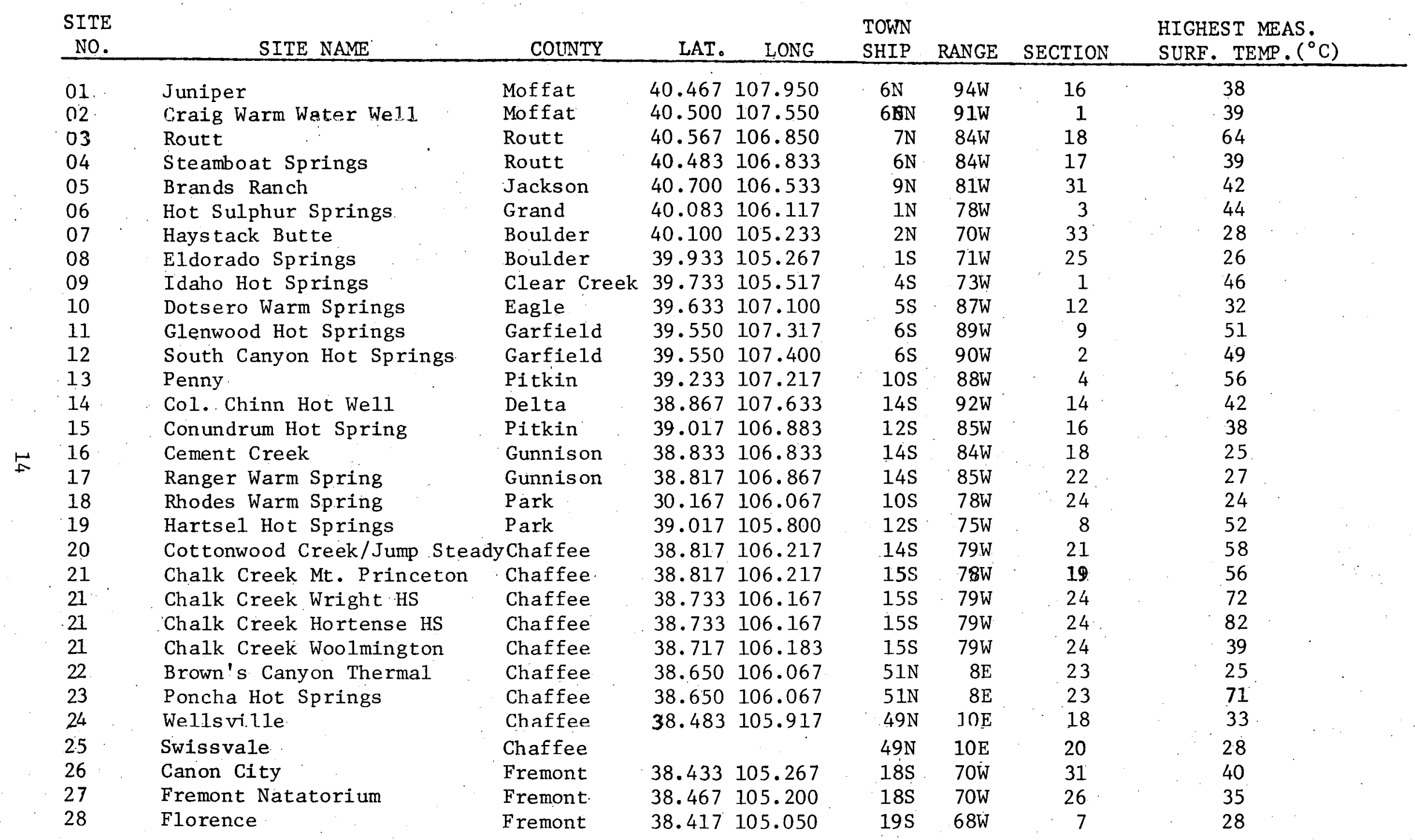


COLORADO HYDROTHERMAL SITES

\begin{tabular}{|c|c|c|c|c|c|c|c|c|c|}
\hline $\begin{array}{l}\text { STTE } \\
\text { NO. }\end{array}$ & SITE NAME & COUNTY & LAT。 & LONG & $\begin{array}{l}\text { TOWN } \\
\text { SHIP } \\
\end{array}$ & RANGE & SECTION & \multicolumn{2}{|c|}{$\begin{array}{l}\text { HIGHEST MEAS. } \\
\text { SURE. TEMP. }\end{array}$} \\
\hline 29 & Don $\mathrm{K}$ Ranch & Fremont & 38.167 & 105.017 & $22 S$ & $68 \mathrm{~W}$ & 5 & & 28 \\
\hline 30 & Clark Artesian We 11 & Pueblo & 38.250 & 104.617 & $21 S$ & $65 \mathrm{~W}$ & 1 & & 25 \\
\hline 31 & Mineral Hot Springs & Saguache & 38.167 & 105.917 & $45 \mathrm{~N}$ & $9 \mathrm{E}$ & 12 & & 60 \\
\hline 32 & Valley View HS & Saguache & 38.200 & 105.817 & $46 \mathrm{~N}$ & $10 \mathrm{E}$ & 36 & & 37 \\
\hline 33 & Shaws Warm Spring & Saguache & 37.750 & 106.317 & $41 N$ & $6 \mathrm{E}$ & 33 & & 30 \\
\hline 34 & Sand Dunes & Alamosa & 37.783 & 105.850 & $41 \mathrm{~N}$ & $10 \mathrm{E}$ & 27 & & 44 \\
\hline 35 & Splashland Hot Water & Alamosa & 37.483 & 105.850 & $38 \mathrm{~N}$ & $10 \mathrm{E}$ & 34 & & 40 \\
\hline 36 & Dexter & Conejos & 37.300 & 105.800 & $35 \mathrm{~N}$ & $11 \mathrm{E}$ & 8 & & 20 \\
\hline 37 & McIntyre & Conejos & & & $35 \mathrm{~N}$ & $11 \mathrm{E}$ & 18 & & 14 \\
\hline 38 & Dutch Crow1ey & Archuleta & 37.033 & 106.800 & $32 \mathrm{~N}$ & $2 E$ & 18 & & 70 \\
\hline 39 & Stinking Spring & Archuleta & & & $32 \mathrm{~N}$ & IE & 2 & & 27 \\
\hline 40 & Eof $\mathrm{f}$ & Archuleta & 37.183 & 106.300 & $34 \mathrm{~N}$ & $1 W$ & 7 & & 39 \\
\hline 41 & * PagosaSp rings & Archuleta & 37.267 & 107.017 & $35 \mathrm{~N}$ & $2 W$ & 13 & & 58 \\
\hline 42 & Rainbow & Mineral & 37.517 & 106.950 & $38 \mathrm{~N}$ & $1 W$ & 9 & & 40 \\
\hline 43 & Wagon Wheel Gap & Mineral & 37.683 & 106.833 & $41 N$ & $1 \mathrm{E}$ & 35 & & 57 \\
\hline 44 & Antelope & Mineral & 37.750 & 107.033 & $40 \mathrm{~N}$ & $2 W$ & 1 & & 32 \\
\hline 45 & Birdsie & Mineral & & & $40 N$ & $2 \mathrm{~W}$ & 14 & & 30 \\
\hline 46 & Waunita Hot Springs & Gunnison & 38.517 & 106.517 & $49 \mathrm{~N}$ & $4 \mathrm{E}$ & 10 , & 11 & 80 \\
\hline 47 & Cebolla & Gunnison & 30.267 & 107.100 & $46 \mathrm{~N}$ & $2 W$ & 4 & & 40 \\
\hline 48 & Orvis Hot Spring & Ouray & 38.133 & 107.733 & $45 \mathrm{~N}$ & $8 \mathrm{~W}$ & 22 & & 52 \\
\hline 49 & Ouray & Ouray & 38.017 & 107.667 & $44 \mathrm{~N}$ & $7 W$ & 31 & & 69 \\
\hline 50 & Lemon & San Migue 1 & 38.017 & 108.050 & $44 \mathrm{~N}$ & $11 \mathrm{~W}$ & 34 & & 33 \\
\hline 51 & Dunton & Dolores & 37.767 & 108.100 & $41 \mathrm{~N}$ & $11 W$ & 32 & & 42 \\
\hline 52 & Geyser & Dolores & & & $40 N$ & $11 W$ & 6 & & 28 \\
\hline 53 & Paradise & Dolores & & & $40 N$ & $12 \mathrm{~W}$ & 1 & & 46 \\
\hline 54 & $\operatorname{Rico}$ & Dolores & 37.700 & 108.033 & $40 N$ & $11 \mathrm{~W}$ & - & & 44 \\
\hline 55 & Pinkerton & La Plata & $37 \cdot 450$ & 107.800 & $37 \mathrm{~N}$ & $9 \mathrm{~W}$ & 25 & & 32 \\
\hline 56 & Tripp/Trimble & La Plata & 37.383 & 107.850 & $36 \mathrm{~N}$ & $9 \mathrm{~W}$ & 10 & & 44 \\
\hline
\end{tabular}

SOURCES: Barrett and Pear1, 1978, except Latitude, longitude: Cuniff and others, 1979

*Potential Site, all others are inferred 


\section{TABLE 2}

ESTIMATED RESERVOIR TEMPERATURES $\left({ }^{\circ} \mathrm{C}\right)$ AND GEOCHEMICAL DATA

- (Adapted from Barrett and Pearl, 1978)

$\begin{array}{ll}q=\text { quartz } & c=\text { chalcedony } \\ a=\text { amorphous } & c r=\text { cristobalite }\end{array}$

\section{Geothermometer Models}

Antelope W.S

Birdsie W.S.

Brands Ranch

Brown ${ }^{\circ}$ s Grotto W.S. 22

Canon City H.S.
Hot Spring
Spring Date Silica Mixing Number Sampled G.T. T. \%
$\mathrm{Na}-\mathrm{K} \quad \mathrm{Na}-\mathrm{K}-\mathrm{Ca}$ G.T. G.T.

Most

Likely

Sub. Temp. Dischar

\begin{tabular}{lll}
83 & $35-52$ & $3 E$ \\
\hline & 35 & $3 E$
\end{tabular}

$4936 \mathrm{cr} \quad 83$

$-$

$\overline{36}^{-}-\overline{35-52}-\overline{15}$

$52 \mathrm{cr}$

$9170 \mathrm{cr} \quad \overline{102}$

- - - -

171

$42-5 \overline{5}$

$80 \mathrm{E}$

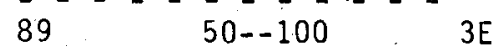

T.D.S.

$42 \mathrm{c} \quad 43 \cdot 1 \mathrm{c} \quad 19$

123

$50--10$

$3 E$

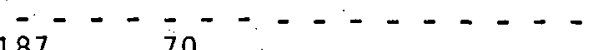

$\begin{array}{ll}35 c \\ 34 c \\ 34 & c\end{array}$

$40 \quad 3$ c 187

$\begin{array}{llll}38 & 12 & \mathrm{C} & 187\end{array}$

68

72

188

$-$

$71 \mathrm{cr} \quad 125 \quad 72 \mathrm{cr} 278 \div 216$

$65 \mathrm{cr} \quad 105 \quad 66 \mathrm{cr} 248 \quad 215$

$78 \mathrm{cr}$

163

$80 \mathrm{cr} 238$

209

$1 / 76$

$82 \mathrm{cr}$

185

$83 \mathrm{cr} .252$

220

$\begin{array}{lllll}73 & \mathrm{cr} & 145 \quad 78 \mathrm{cr} & 249 & 217\end{array}$

$74 \mathrm{cr}$

$14376 \mathrm{cr} 250$

217

$5361 \mathrm{c} 232$

$27 \quad 0 \quad \mathrm{c} \quad 225$

$27.0 \mathrm{c} \quad 225$

296 c 238

$238 \quad 49$

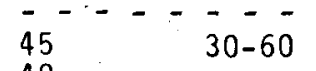

45
48

48
46

49

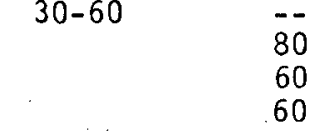

$\begin{array}{ll}5 & 1,230 \\ 1 & 1,220 \\ 2 & 1,210\end{array}$

494

262

\section{Chalk Creek H.S. Area:}

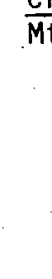

Mt. Princeton

H.S. "A"

21

$7 / 75$
$10 / 75$

$1 / 76$

$4 / 7.6$

H.S. "F"

Hortense H.S.

$21 \quad 7 / 75 \quad 107 \quad \mathrm{q}$

$21 \quad 7 / 75 \quad 118 \mathrm{q}$

$4 / 76 \quad 129 q$

$\begin{array}{ll}110 & q \\ 108 & 9 \\ 105 & 9\end{array}$

$\begin{array}{ll}105 & q \\ 127 & q\end{array}$

$\begin{array}{llll}194 \quad 78 & q & 149\end{array}$

$\begin{array}{llll}190 & 77 & 9 & 148\end{array}$

$\begin{array}{llll}186 & 77 & 9 & 151\end{array}$

$\begin{array}{llll}236 & 81 & 9 & 150\end{array}$

20181 q 150

164. 57 q 146

$\begin{array}{llll}156 & 54 & q & 144\end{array}$

$\begin{array}{llll}164 & 56 & 9 & 141\end{array}$

$\begin{array}{llll}186 & 61 & 9 & 145\end{array}$

1189

$164 \quad 56 \quad q \quad 144$

80

$150-200$

47

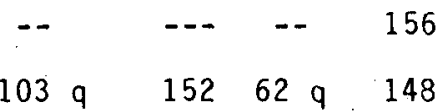

$21 \quad 8 / 75$

$8 / 75$

$7 / 75$

$\begin{array}{lllll}116 q & 172 \quad 64 q & 145\end{array}$

56
58
58
59

$150-200$

94

93

93

$150-200$

62

$150-200$

77
$10 / 75$

$1 / 76 \quad 120 \mathrm{q}$

Water Well

245

248

244

248

$\begin{array}{llll}51 & 150-200 & 12 & 229\end{array}$

340

336

351

341

\section{Wr}

Wright Hot Well(W.)

143

234

$150--200$

313 
TABLE 2 CONT.

Spring Date Silica Mixing Model. Na-K Na-K-Ca Lost Likely Discharge T.D.S. Hot Spring Number Sampled G.T. I. \% G.T. G.T. Sub.Temp. gpm. Chalk Creek Area Cont.

\begin{tabular}{|c|c|c|c|c|c|c|c|c|c|c|c|c|c|}
\hline Young Life Hot Well & 21 & $7 / 75$ & 116 & $q$ & 188 & 71 & $q$ & 135 & 68 & & $150-200$ & -- & 259 \\
\hline Clark Artesian Well & 30 & $9 / 75$ & 40 & $q$ & 61 & 65 & $q$ & 280 & 159 & & $25-50$ & 12 & 1,210 \\
\hline$\frac{\text { Colonel Chinn Hot }}{\text { Water Well }}$ & 14 & $4 / 76$ & 41 & c & 43 & & $c$ & 183 & 170 & & -- & -- & \\
\hline Conundrum H.S. & 15 & $9 / 75$ & 40 & $\mathrm{cr}$ & 41 & 6 & $\mathrm{cr}$ & 187 & -4 & & $\begin{array}{r}40-50 \\
-\quad---\end{array}$ & $\begin{array}{r}50 \\
-\end{array}$ & 1,91 \\
\hline$\frac{\text { Cottonwood H.S. Area }}{\text { Cottonwood H.S. }}$ & $\dot{\Xi}_{20}$ & $6 / 75$ & 110 & $q$ & 174 & 70 & $q$ & 132 & 84 & & $105-182$ & $10 E$ & \\
\hline Jumpsteady H.S. & 20 & $\begin{array}{r}6 / 75 \\
10 / 75 \\
1 / 76 \\
4 / 76\end{array}$ & $\begin{array}{r}108 \\
105 \\
109 \\
--\end{array}$ & $\begin{array}{l}q \\
q \\
q\end{array}$ & $\begin{array}{l}180 \\
174 \\
182 \\
---\end{array}$ & $\begin{array}{l}74 \\
74 \\
74 \\
--\end{array}$ & $\begin{array}{l}q \\
q \\
q\end{array}$ & $\begin{array}{l}133 \\
131 \\
131 \\
135\end{array}$ & $\begin{array}{l}79 \\
85 \\
83 \\
83\end{array}$ & & $105-182$ & $\begin{array}{l}-- \\
90 \\
50 \\
50\end{array}$ & \\
\hline $\begin{array}{l}\text { Merrifield Hot } \\
\text { Water Well }\end{array}$ & 20 & $6 / 75$ & 97 & $q$ & 174 & 77 & $q$ & 141 & $\begin{array}{r}68 \\
--\end{array}$ & & $105-182$ & -- & \\
\hline$\frac{\text { Craig Warm Water }}{\text { Well }}$ & 2 & $1 / 76$ & 58 & $q$ & $\begin{array}{l}70 \\
35\end{array}$ & $\begin{array}{l}50 \\
20\end{array}$ & $\begin{array}{l}q \\
c\end{array}$ & 100 & 104 & & $40-70$ & 24 & \\
\hline Dexter W.S. & 36 & $4 / 76$ & -- & & 19 & 36 & a. & $2 \overline{7} 8$ & $\begin{array}{r}-\overrightarrow{9} 1 \\
- \\
-\end{array}$ & & $\begin{array}{l}-\overline{-}-\overline{-} \\
-20-50 \\
---\end{array}$ & $-{ }^{-} 50 E$ & \\
\hline$\frac{\text { Don K. Ranch }}{\text { Artesian Well }}$ & 29 & $9 / 75$ & 42 & $\mathrm{cr}$ & 63 & 61 & $\mathrm{cr}$ & 219 & 190 & & $\ldots$ & $-\quad 25$ & 1,7 \\
\hline Dotsero W.S. & 10 & $\begin{array}{l}9 / 75 \\
1 / 76 \\
4 / 76\end{array}$ & $\begin{array}{l}\overrightarrow{16} \\
16\end{array}$ & & $\begin{array}{c}-- \\
27 \\
29\end{array}$ & $\begin{array}{l}-5 \\
36\end{array}$ & $\begin{array}{l}\text { c } \\
\text { c }\end{array}$ & $\begin{array}{l}104 \\
135 \\
104\end{array}$ & $\begin{array}{l}113 \\
144 \\
112\end{array}$ & & $32-45$ & $\begin{array}{l}500 E \\
525 E \\
800 E\end{array}$ & $\begin{array}{r}10,4 \\
9,9\end{array}$ \\
\hline S. Dotsero W.S. & 10 & $12 / 75$ & 160 & & 29 & 26 & c & $102^{-}$ & 109 & & $32-45$ & $\overline{1}, \overline{0} 00 \mathrm{E}$ & 9,0 \\
\hline Dunton H.S. & 51 & $\begin{array}{l}9 / 75 \\
1 / 76 \\
4 / 76\end{array}$ & $\begin{array}{l}54 \\
51 \\
53\end{array}$ & $\begin{array}{l}c \\
c \\
c\end{array}$ & $\begin{array}{l}69 \\
65 \\
69\end{array}$ & $\begin{array}{l}40 \\
39 \\
43\end{array}$ & $\begin{array}{l}c \\
c \\
c\end{array}$ & $\begin{array}{l}329 \\
328 \\
342\end{array}$ & $\begin{array}{l}50 \\
47 \\
52\end{array}$ & & $50-70$ & $\begin{array}{l}26 \\
25 \\
25\end{array}$ & $\begin{array}{l}1,26 \\
1,34 \\
1,30\end{array}$ \\
\hline
\end{tabular}

Dutch Crowley

Eldorado Springs

Spring "A

$39 \quad 8 / 76$

$63 \mathrm{c}$

$\begin{array}{llll}65 & 7 & \mathrm{c} & 271\end{array}$

16

$70-80$

$75 \mathrm{E}$

Spring "B"

$8 \quad 9 / 75$

$23 \mathrm{c}$

$\begin{array}{llll}27 & 8 & c & 314\end{array}$

43

$26-40$

101

$8 \quad 9 / 75$

$21 \mathrm{c}$

$\begin{array}{llll}26 & 10 \mathrm{c} & 320\end{array}$

$\begin{array}{llllll}21 & c & 26 & 19 & c & 254\end{array}$

45

$26-40$

$4 / 76$

$26 \quad 1$ c 311

46

$5938 \mathrm{cr} \quad \overline{2} 2 \overrightarrow{1}$

$5 \overline{6}$

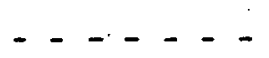

Eoff Artesian Well $40.8 / 76$

$47 \mathrm{cr}$

40-60

81

Florence Artesian

Well (Penrose)

$28 \quad 9 / 75: 34 \mathrm{c}$

$4140 \mathrm{c}$

$212 \quad 178$

34-50

- 130

1,480

Freemont Natatorium H.S.

$27 \quad 9 / 75$

$1 / 76$

$23 \mathrm{c}$

$-$

$-$

- - . - . -

$21 \mathrm{c}$

$3223 \mathrm{c}$

172

72
73

$35-50$

$20 \quad 1,370$

$4 / 76$

$32 \quad 23 \mathrm{c}$

$171 \quad 71$

$\begin{array}{ll}20 & 1,300 \\ 18 & 1,330\end{array}$

Geyser W.S.

$58 \mathrm{c}$

$11380 \mathrm{c}$

$183--\overline{160}$

60-120 25-200E

1,620 
TABLE 2 CONT.

Hot Spring Number Sampled G.T. I. \% G.T. G.T. Sub.Temp. gpm.

Glenwood Springs Area:

\begin{tabular}{|c|c|c|c|c|c|c|c|c|c|c|c|c|}
\hline Big Spring & 11 & $7 / 75$ & 51 & & 59 & 18 & c & 133 & 148 & -- & 2,263 & 20,200 \\
\hline Drinking Spring & 11 & $\begin{array}{r}7 / 75 \\
10 / 75 \\
1 / 76 \\
4 / 76\end{array}$ & $\begin{array}{l}51 \\
47 \\
48 \\
48\end{array}$ & $\begin{array}{l}c \\
c \\
c \\
c\end{array}$ & $\begin{array}{l}59 \\
49 \\
51 \\
51\end{array}$ & $\begin{array}{r}18 \\
3 \\
0 \\
0\end{array}$ & $\begin{array}{l}c \\
c \\
c \\
c\end{array}$ & $\begin{array}{l}133 \\
131 \\
168 \\
135\end{array}$ & $\begin{array}{l}147 \\
145 \\
186 \\
149\end{array}$ & -- & $\begin{array}{r}-- \\
\overline{161} \\
140\end{array}$ & $\begin{array}{l}20,300 \\
20 ; 200 \\
20,500 \\
18,800\end{array}$ \\
\hline $\begin{array}{l}\text { Yapor Caves, Men }{ }^{\circ} s \\
\text { H.S. }\end{array}$ & 11 & $9 / 75$ & 45 & c & 49 & 3 & c & 129 & 143 & $\because$ & $5 \mathrm{E}$ & $18 ; 000$ \\
\hline Graves Spring & 11 & $9 / 75$ & 51 & c & 77 & 46 & $c$ & 133 & 144 & -- & 5 & 21,500 \\
\hline Spring "A" & 11 & $7 / 75$ & 48 & c & 73 & 46 & c & 134 & 149 & -- & $2-3 E$ & 17,600 \\
\hline Spring "B" & 11 & $\begin{array}{r}7 / 75 \\
10 / 75 \\
1 / 76 \\
4 / 76\end{array}$ & $\begin{array}{l}48 \\
44 \\
45 \\
45\end{array}$ & $\begin{array}{l}c \\
c \\
c \\
c \\
c\end{array}$ & $\begin{array}{l}51 \\
47 \\
49 \\
49\end{array}$ & $\begin{array}{l}0 \\
9 \\
6 \\
6\end{array}$ & $\begin{array}{l}c \\
c \\
c \\
c\end{array}$ & $\begin{array}{l}135 \\
131 \\
133 \\
135\end{array}$ & $\begin{array}{l}149 \\
145 \\
165 \\
151\end{array}$ & -- & $\begin{array}{r}75 \\
75 \\
100 \\
110\end{array}$ & $\begin{array}{l}18,300 \\
18,400 \\
17,700 \\
17,800\end{array}$ \\
\hline Spring "D" & 11 & $7 / 75$ & 48 & c & 51 & 2 & c & 133 & 147 & -- & 74 & 18,000 \\
\hline Railroad Spring & 11 & $\begin{array}{l}1 / 76 \\
4 / 76\end{array}$ & $\begin{array}{l}47 \\
47\end{array}$ & $\begin{array}{l}c \\
c\end{array}$ & $\begin{array}{l}49 \\
49\end{array}$ & $\begin{array}{l}6 \\
6\end{array}$ & $\begin{array}{l}c \\
c\end{array}$ & $\begin{array}{l}143 \\
138\end{array}$ & $\begin{array}{l}158 \\
152\end{array}$ & -- & $\begin{array}{l}75 \\
75\end{array}$ & $\begin{array}{l}18,400 \\
18,200\end{array}$ \\
\hline
\end{tabular}

$\frac{\text { Hartsel Hot Springs }}{\text { Spring "A" }}$

$19 \quad 6 / 75 \quad 63$ c $\quad 35 \quad 44$ c $\quad 162 \quad 152$

Spring "B"

$19 \begin{array}{rr}6 / 75 & 59 c \\ 10 / 75 & 55 c \\ 1 / 76 & 56 c \\ 4 / 76 & 58 c\end{array}$

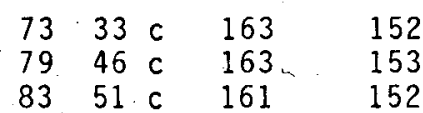

$87 \quad 53$ c $163 \quad 153$

$\begin{array}{ll}- & 2,280 \\ - & 2,140 \\ 40 & 2,260 \\ 48 & 2,310 \\ 50 & 2,330\end{array}$

Haystack Butte

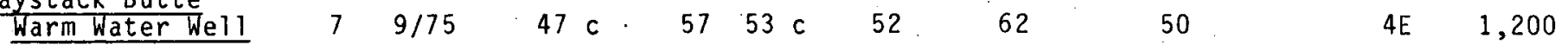

Hot Sulphur Springs

Spring "A"

$\begin{array}{r}6 / 75 \\ 10 / 75 \\ 1 / 76 \\ 4 / 76 \\ \hline\end{array}$

$\begin{array}{llllllllll}86 & q & 109 & 63 \cdot q & 169 & 171 & 75-150 & -- & 1,200\end{array}$

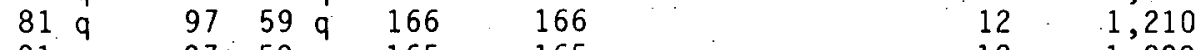

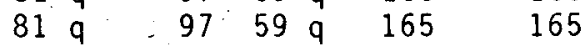

$\begin{array}{lllllll}84 & \mathrm{q} & 103 & 64 & \mathrm{q} & 169 & 168\end{array}$

121,220

\begin{tabular}{|c|c|c|c|c|c|c|c|c|c|c|c|c|}
\hline Spring "B" & 6 & $7 / 75$ & 86 & & 113 & 67 & & 169 & 169 & $75-150$ & 1 & 1,200 \\
\hline Spring "C" & 6 & $\begin{array}{r}7 / 75 \\
10 / 75\end{array}$ & $\begin{array}{l}86 \\
81\end{array}$ & & $\begin{array}{r}115 \\
99\end{array}$ & $\begin{array}{l}69 \\
64\end{array}$ & $q r$ & $\begin{array}{l}170 \\
165\end{array}$ & $\begin{array}{l}170 \\
164\end{array}$ & $75-150$ & $\begin{array}{r}3 \\
15\end{array}$ & $\begin{array}{l}1,210 \\
1,190\end{array}$ \\
\hline Spring "D" & 6 & $10 / 75$ & 80 & $q$ & 97 & 63 & $\mathrm{q}$ & 167 & 166 & $\begin{array}{l}75-150 \\
-\ldots-\end{array}$ & 23 & 1,190 \\
\hline$\frac{\text { Idaho Hot Springs }}{\text { Spring "A" }}$ & 9 & $\begin{array}{r}7 / 75 \\
10 / 75 \\
2 / 76 \\
4 / 76\end{array}$ & $\begin{array}{l}66 \\
59 \\
71 \\
78\end{array}$ & $\begin{array}{l}c r \\
c r \\
c r \\
c r\end{array}$ & $\begin{array}{r}109 \\
95 \\
141 \\
171\end{array}$ & $\begin{array}{l}64 \\
63 \\
76 \\
81\end{array}$ & $\begin{array}{l}c r \\
c r \\
c r \\
c r\end{array}$ & $\begin{array}{l}231 \\
231 \\
225 \\
228\end{array}$ & $\begin{array}{l}210 \\
210 \\
204 \\
207\end{array}$ & -- & $\begin{array}{l}21 \\
-- \\
--\end{array}$ & $\begin{array}{l}2,020 \\
2,110 \\
1,950 \\
1,940\end{array}$ \\
\hline Spring "B" & 9 & $7 / 75$ & 66 & $\mathrm{cr}$ & - & -- & & 230 & 210 & $\cdots$ & - & 2,070 \\
\hline Spring "C" & 9 & $7 / 75$ & 47 & $\mathrm{cr}$ & -- & -- & & 235 & 206 & - & 1 & 1,070 \\
\hline Lodge Well & 9 & $10 / 75$ & 59 & $\mathrm{cr}$ & 81 & 48 & $\mathrm{cr}$ & 231 & 210 & -- & 30 & 2,070 \\
\hline
\end{tabular}


TABLE 2 CONT.

\begin{tabular}{l}
$\begin{array}{l}\text { Spring } \\
\text { Number } \\
\text { Sampled Silica }\end{array}$ G.T. \\
\hline
\end{tabular}

$\begin{array}{rrr}1 & 7 / 75 & 53 \mathrm{c} \\ 10 / 75 & 47 \mathrm{c} \\ & 1 / 76 & 50 \mathrm{c} \\ 4 / 76 & 51 \mathrm{c}\end{array}$

\section{Juniper H.S.}

50

Lemon H.S.

$1 / 76$

15 a

$4 / 76 \quad 14$ a

McIntyre W.S.

$37 \quad 4 / 76$

Mineral Hot Springs

$6 / 75$
$10 / 75$
$1 / 76$
$4 / 76$

$8159 \mathrm{c}$

$7361 \mathrm{c}$

75
67

67
.70

80

76

$8161 \mathrm{c}$

69

78

$50-75$

gpm.

1,150

$14 \quad 1,160$

$13 \quad 1,160$

- . . . . . . . . .

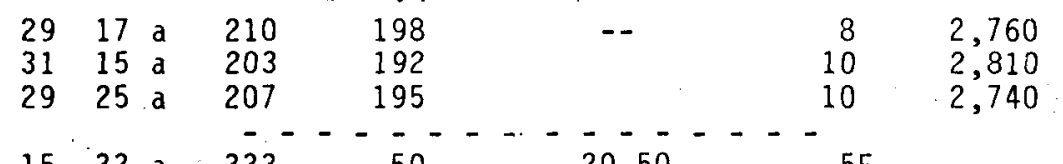

1533 a 333

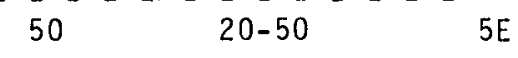

$87 \quad 38$ c 206

206

$\begin{array}{llll}79 & 30 \mathrm{c} & 202\end{array}$

90
90
89
90

$70-90$

100

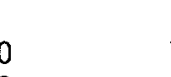

$-$

Spring "C"
Spring "D"

$316 / 75-72 c$

$67 \mathrm{c}$

$83 \cdot 34$ c 199

$69 \mathrm{c}$

$83 \cdot 34$ c 202

$93 \quad 43$ C $\quad 197 \quad 91$

$31 \quad 6 / 75 \quad 70 \mathrm{c}$

$10 / 75 \quad 67 \mathrm{c}$

$1 / 76 \quad 68 \mathrm{c}$

$\begin{array}{lllll}89 & 41 \text { c } & 202 & 92\end{array}$

$\begin{array}{llll}79 & 30 & \mathrm{c} & 198\end{array}$

$\begin{array}{llll}81 & 32 & c & 195\end{array}$

$83 \quad 34$ c 202

Orvis H.S.

$\begin{array}{lll}48 & 9 / 75 & 73 \mathrm{c} \\ & 1 / 76 & 82 \mathrm{c} \\ & 4 / 76 & 75 \mathrm{c}\end{array}$

$9954 \mathrm{c} \quad 179^{-}$

$\begin{array}{llll}127 & 66 & \mathrm{c} & 183\end{array}$

$\begin{array}{llll}107 & 54 & \mathrm{c} & 187\end{array}$

$187 \quad 93$

92
91
87
90

90

Ouray Hot Springs

Wiesbaden Vapor
Caves "A"

Wiesbaden "Vapor

Wiesbaden Vapor
Caves "C"

Pool H.S.

Uncompahgre H.S.

Pagosa Spgs,

Big Spg

Courthouse hot

water well

Spa Hot Water Well

Paradise Hot Spring
Caves "B"

$61 \mathrm{c}$

$\begin{array}{llll}51 \quad 4 & c & 196\end{array}$

$47 \mathrm{c}$

11175 c 198

32

32

70-90

$70-90$

$70--90$

$$
\begin{aligned}
& 28 \\
& 41 \\
& 43
\end{aligned}
$$

$70-90$

170

95

70-90

$-$

723

- 665

$\begin{array}{ll}- & 690 \\ -5 \mathrm{E} & 657\end{array}$

$--$

648

$\begin{array}{ll}-1 & 2,270 \\ -1 & 2,490\end{array}$

$-$

$-1 \quad 2,270$
8175
$10 / 75$
$1 / 76$
$4 / 76$

$41 \quad 8 / 75$

$41 \quad 8 / 75$

$8 / 75$

$53 \quad 9 / 75$

$1 / 76$

$4 / 76$

49

$9 / 75$
$1 / 76$
$4 / 76$

$69 \mathrm{c}$

$71 \mathrm{c}$

$71 \mathrm{C}$

$\begin{array}{llll}77 & 16 & \mathrm{c} & 191\end{array}$

$\begin{array}{llll}79 & 15 & \mathrm{c} & 184\end{array}$

$\begin{array}{llll}79 & 15 & \mathrm{c} & 192\end{array}$

39

39

$\begin{array}{llllll}66 \text { c } & 109 & 58 \text { c } & 192 & 40\end{array}$

192

$76 \mathrm{c}$

$80 \mathrm{c}$

11354 c 209

-- - 209

13364 c 207

$81 \mathrm{c}$

139.66 c 210

194

191

193

74 c $\quad 113 \quad 56$ c $\quad 210 \quad 193$

$73 \mathrm{c}$

39 a

56 a

39 a $\begin{array}{ll}45 & 4 \\ 53 & 7 \\ 43 & 1\end{array}$ $247---\overline{252}$

247
247

247
$245 \quad 250$
11760 c $211 \quad 195$

$\begin{array}{rrr}70--90 & 1 E & 1,380 \\ & 30 E & 1,430 \\ . . & 5 E & 1,390 \\ 70-90 & 125 & 1,650 \\ & 60 & 1,660 \\ & 200 & 1,640\end{array}$

$70-90 \quad 5 \quad 1,570$

$30-150$

265

226

241

260

3.200

$3, \overline{310}$

3,040

3,300

3,320

6,070

6,530

6,180 
TABLE 2 CONT.

\begin{tabular}{|c|c|c|c|c|c|c|c|c|c|c|c|c|}
\hline Hot Spring & & $\begin{array}{l}\text { Date } \\
\text { Sampled }\end{array}$ & $\begin{array}{l}\text { Sili } \\
\text { G.T. }\end{array}$ & & $\begin{array}{l}\text { Mixi } \\
\text { Mod } \\
\text { T. }\end{array}$ & $\%$ & & $\begin{array}{l}\mathrm{Na}-\mathrm{K} \\
\text { G.T. }\end{array}$ & $\begin{array}{c}\mathrm{Na}-\mathrm{K}-\mathrm{Ca} \\
\mathrm{G} . \mathrm{T} . \\
\end{array}$ & $\begin{array}{r}\text { Most } \\
\text { Likely } \\
\text { Sub. Temp. } \\
\end{array}$ & $\begin{array}{l}\text { Discharge } \\
\text { gpm. }\end{array}$ & $\begin{array}{l}\text { T.D.S. } \\
\mathrm{mg} / \mathrm{l}\end{array}$ \\
\hline Penny Hot Springs & 13 & $\begin{array}{l}9 / 75 \\
1 / 76 \\
4 / 76\end{array}$ & $\begin{array}{r}15 \\
3 \\
39\end{array}$ & $\begin{array}{l}a \\
a \\
a\end{array}$ & $\begin{array}{l}35 \\
3.5 \\
45\end{array}$ & $\begin{array}{r}25 \\
48 \\
2\end{array}$ & $\begin{array}{l}a \\
a \\
a\end{array}$ & $\begin{array}{l}199 \\
197 \\
202\end{array}$ & $\begin{array}{l}93 \\
89 \\
92\end{array}$ & $60-90$ & $\begin{array}{l}10 \\
10 \\
10\end{array}$ & $\begin{array}{l}2,820 \\
2,820 \\
2,750\end{array}$ \\
\hline Granges Spring & 13 & $1 / 76$ & 7 & a & 41 & 50 & a & 198 & 90 & $60-90$ & 12 & 2,960 \\
\hline$\frac{\text { Pinkerton H.S. Area: }}{\text { Spring "A" }}$ & 55 & $\begin{array}{l}9 / 75 \\
1 / 76 \\
4 / 76\end{array}$ & $\begin{array}{l}78 \\
78 \\
78\end{array}$ & $\begin{array}{l}q \\
q \\
q\end{array}$ & $\begin{array}{l}127 \\
127 \\
133\end{array}$ & $\begin{array}{l}81 \\
81 \\
82\end{array}$ & $\begin{array}{l}q \\
q \\
q\end{array}$ & $\begin{array}{l}231 \\
231 \\
234\end{array}$ & $\begin{array}{l}205 \\
202 \\
206\end{array}$ & $75-125$ & $\begin{array}{l}54 \\
54 \\
54\end{array}$ & $\begin{array}{l}3,990 \\
3,880 \\
3,770\end{array}$ \\
\hline Spring "B". & 55 & $9 / 75$ & -- & & -- & -- & & 234 & 206 & $75-125$ & 20 & $-\ldots$ \\
\hline Mound Spring & 55 & $\begin{array}{l}9 / 75 \\
1 / 76 \\
4 / 76\end{array}$ & $\begin{array}{l}79 \\
78 \\
78\end{array}$ & & $\begin{array}{l}139 \\
137 \\
137\end{array}$ & $\begin{array}{l}84 \\
85 \\
85\end{array}$ & $\begin{array}{l}q \\
q \\
q\end{array}$ & $\begin{array}{l}234 \\
235 \\
235\end{array}$ & $\begin{array}{l}206 \\
206 \\
207\end{array}$ & $75-125$ & $\begin{array}{l}8 E \\
5 E \\
5 E\end{array}$ & $\begin{array}{l}3,940 \\
3,880 \\
3,840\end{array}$ \\
\hline$\frac{\text { Poncha Hot Springs }}{\text { Spring "A" }}$ & 23 & $\begin{array}{r}6 / 75 \\
10 / 75 \\
1 / 76 \\
4 / 76\end{array}$ & $\begin{array}{l}126 \\
119 \\
137 \\
137\end{array}$ & $\begin{array}{l}q \\
q \\
q \\
q\end{array}$ & $\begin{array}{l}173 \\
157 \\
201 \\
201\end{array}$ & $\begin{array}{l}63 \\
60 \\
69 \\
69\end{array}$ & $\begin{array}{l}q \\
q \\
q \\
q\end{array}$ & $\begin{array}{l}155 \\
154 \\
154 \\
159\end{array}$ & $\begin{array}{r}99 \\
140 \\
141 \\
145\end{array}$ & $115-145$ & $\begin{array}{l}-- \\
\overline{-} \\
200\end{array}$ & $\begin{array}{l}667 \\
678 \\
697 \\
654\end{array}$ \\
\hline Spring "B" & 23 & $6 / 75$ & 127 & $q$ & 183 & 68 & $q$ & 154 & 139 & $115-145$ & $30 E$ & 655 \\
\hline Spring "C" & 23 & $\begin{array}{r}6 / 75 \\
10 / 75 \\
1 / 76 \\
4 / 76\end{array}$ & $\begin{array}{l}126 \\
119 \\
130 \\
136\end{array}$ & & $\begin{array}{l}185 \\
169 \\
195 \\
209\end{array}$ & $\begin{array}{l}70 \\
68 \\
72 \\
73\end{array}$ & $\begin{array}{l}q \\
q \\
q \\
q\end{array}$ & $\begin{array}{l}157 \\
156 \\
154 \\
158\end{array}$ & $\begin{array}{r}96 \\
142 \\
141 \\
144\end{array}$ & $115-145$ & $\begin{array}{l}2 \\
3 \\
2 \\
4\end{array}$ & $\begin{array}{l}670 \\
660 \\
685 \\
655\end{array}$ \\
\hline Rainbow Hot Spring & 42 & $9 / 75$ & 41 : & $c r$ & 41 & 0 & $\mathrm{cr}$ & $\overline{68}^{-}$ & $-\overline{2}^{-}$ & $40-50$ & 45 & 161 \\
\hline$\frac{\text { Ranger Warm Spring }}{-}$ & 17 & $\begin{array}{r}7 / 75 \\
10 / 75 \\
1 / 76 \\
4 / 76\end{array}$ & $\begin{array}{l}32 \\
28 \\
30 \\
30\end{array}$ & $\begin{array}{l}\mathrm{c} \\
\mathrm{c} \\
\mathrm{c} \\
\mathrm{c}\end{array}$ & $\begin{array}{l}67 \\
29 \\
45 \\
45\end{array}$ & $\begin{array}{r}71 \\
1 \\
49 \\
49\end{array}$ & $\begin{array}{l}c \\
c \\
c \\
c\end{array}$ & $\begin{array}{l}214^{-} \\
216 \\
218 \\
217\end{array}$ & $\begin{array}{l}-56 \\
66 \\
60 \\
60 \\
-\quad-\end{array}$ & $30-60$ & $\begin{array}{l}132 \\
250 \mathrm{E} \\
225 \mathrm{E} \\
175 \mathrm{E}\end{array}$ & $\begin{array}{l}461 \\
465 \\
466 \\
474\end{array}$ \\
\hline Rhodes W.S. & 18 & $\begin{array}{r}6 / 75 \\
10 / 75\end{array}$ & $\begin{array}{l}10 \\
13\end{array}$ & $\begin{array}{l}c \\
c\end{array}$ & $\begin{array}{l}21 \\
23\end{array}$ & $\begin{array}{l}65 \\
41\end{array}$ & $\begin{array}{l}c \\
c\end{array}$ & $\begin{array}{l}240 \\
222 \\
-\end{array}$ & $\begin{array}{c}2 \\
10 \\
----\end{array}$ & $25-35$ & 200 & $\begin{array}{l}186 \\
194\end{array}$ \\
\hline$\frac{\text { Rico }}{\text { Diamond Drill Hole }}$ & 54 & $1 / 76$ & 26 & a & 39 & 18 & $\mathrm{a}$ & 307 & 56 & -- & 15 & 2,250 \\
\hline Big Geyser W.S. & 54 & $\begin{array}{l}9 / 75 \\
4 / 76\end{array}$ & $\begin{array}{l}22 \\
35\end{array}$ & $\begin{array}{l}\mathrm{a} \\
\mathrm{a}\end{array}$ & $\begin{array}{l}31 \\
37\end{array}$ & $\begin{array}{r}19 \\
1\end{array}$ & $\begin{array}{l}\mathrm{a} \\
\mathrm{a}\end{array}$ & $\begin{array}{l}297 \\
315\end{array}$ & $\begin{array}{l}57 \\
56\end{array}$ & -- & $\begin{array}{r}8 \\
12\end{array}$ & $\begin{array}{l}2,750 \\
2,740\end{array}$ \\
\hline Geyser W.S. & 54 & $9 / 75$ & 22 & $a$ & 35 & 15 & $a$ & 301 & 59 & -- & 14 & 2,790 \\
\hline Little Spring & 54 & $\begin{array}{l}9 / 75 \\
1 / 76\end{array}$ & $\begin{array}{l}26 \\
26\end{array}$ & $\begin{array}{l}\mathrm{a} \\
\mathrm{a}\end{array}$ & $\begin{array}{l}35 \\
37\end{array}$ & $\begin{array}{l}15 \\
10\end{array}$ & $\begin{array}{l}a \\
a\end{array}$ & $\begin{array}{l}305 \\
185 \\
-\quad-\end{array}$ & $\begin{array}{r}58 \\
17 \\
-\quad-\end{array}$ & $\begin{array}{c}-- \\
----\end{array}$ & $\begin{array}{r}13 \\
-\quad 15 \\
-\end{array}$ & $\begin{array}{l}2,790 \\
2,700\end{array}$ \\
\hline$\frac{\text { Routt Hot Springs }}{\text { Spring "A" }}$ & 3 & $\begin{array}{r}7 / 75 \\
10 / 75 \\
1 / 76 \\
4 / 76\end{array}$ & $\begin{array}{l}136 \\
125 \\
129 \\
131\end{array}$ & $\begin{array}{l}q \\
q \\
q \\
q\end{array}$ & $\begin{array}{l}225 \\
199 \\
209 \\
213\end{array}$ & $\begin{array}{l}75 \\
71 \\
73 \\
73\end{array}$ & $\begin{array}{l}q \\
q \\
q \\
q\end{array}$ & $\begin{array}{l}170 \\
165 \\
167 \\
169\end{array}$ & $\begin{array}{l}154 \\
154 \\
155 \\
157\end{array}$ & $125-175$ & $\begin{array}{l}33 \\
50 \\
25 \\
35\end{array}$ & $\begin{array}{l}552 \\
518 \\
521 \\
527\end{array}$ \\
\hline Spring "B" & 3 & $7 / 75$ & 136 & $q$ & 231 & 76 & $q$ & 170 & $\begin{array}{r}159 \\
-\quad--\end{array}$ & $\begin{array}{r}125-175 \\
-\ldots-\ldots\end{array}$ & -30 & 539 \\
\hline Sand Dunes Hot Well & 34 & $8 / 75$ & 26 & $\dot{a}$ & 39 & 19 & $a$ & 205 & $\begin{array}{l}187 \\
-\quad--\end{array}$ & -- & -- & 334 \\
\hline
\end{tabular}


TABLE 2 CONT.

\begin{tabular}{|c|c|c|c|c|c|c|c|c|c|c|c|c|c|}
\hline Hot Spring & $\begin{array}{l}\text { ing } \\
\text { ber }\end{array}$ & $\begin{array}{l}\text { Date } \\
\text { Sampled }\end{array}$ & $\begin{array}{l}\text { Sili } \\
\text { G.T. }\end{array}$ & & $\begin{array}{l}\text { Mixi } \\
\text { Mod } \\
\text { T. }\end{array}$ & $\%$ & & $\begin{array}{l}\mathrm{Na}-\mathrm{K} \\
\mathrm{G} . \mathrm{T} .\end{array}$ & $\begin{array}{l}\mathrm{Na}-\mathrm{K}-\mathrm{Ca} \\
\therefore \mathrm{G} . \mathrm{T} .\end{array}$ & & $\begin{array}{c}\text { Most } \\
\text { Likely } \\
\text { Sub. Temp. }\end{array}$ & $\begin{aligned} & \text { Discharge } \\
&-\quad \mathrm{gpm} . \\
&\end{aligned}$ & $\begin{array}{r}\text { T.D.S. } \\
\mathrm{mg} / \mathrm{l}\end{array}$ \\
\hline Shaws W.S. & 33 & $\begin{array}{r}8 / 75 \\
10 / 75 \\
1 / 76 \\
4 / 76\end{array}$ & $\begin{array}{r}8 \\
2 \\
17 \\
4\end{array}$ & $\begin{array}{l}a \\
a \\
a \\
a\end{array}$ & $\begin{array}{l}26 \\
26 \\
28 \\
26\end{array}$ & $\begin{array}{l}32 \\
32 \\
19 \\
32\end{array}$ & $\begin{array}{l}\mathrm{a} \\
\mathrm{a} \\
\dot{a} \\
\mathrm{a}\end{array}$ & $\begin{array}{r}101 \\
98 \\
101 \\
100\end{array}$ & $\begin{array}{r}103 \\
104 \\
83 \\
102\end{array}$ & & $30-60$ & $\begin{array}{l}34 \\
34 \\
52 \\
40\end{array}$ & $\begin{array}{l}406 \\
402 \\
424 \\
398\end{array}$ \\
\hline$\frac{\text { South Canyon } H_{\text {. S }}}{\text { Spring "A" }}$ & 12 & $\begin{array}{r}7 / 75 \\
10 / 75 \\
1 / 76 \\
4 / 76\end{array}$ & $\begin{array}{l}66 \\
60 \\
67 \\
63\end{array}$ & $\begin{array}{l}\mathrm{c} \\
\mathrm{c} \\
\mathrm{c} \\
\mathrm{c}\end{array}$ & $\begin{array}{l}123 \\
103 \\
127 \\
115\end{array}$ & $\begin{array}{l}67 \\
60 \\
68 \\
65\end{array}$ & $\begin{array}{l}\mathrm{c} \\
\mathrm{c} \\
\mathrm{c} \\
\mathrm{c}\end{array}$ & $\begin{array}{l}138 \\
137 \\
140 \\
140\end{array}$ & $\begin{array}{l}137 \\
135 \\
137 \\
137\end{array}$ & & $100-130$ & $\begin{array}{r}12 \\
7 \\
9 \\
17\end{array}$ & $\begin{array}{l}794 \\
800 \\
783 \\
772\end{array}$ \\
\hline Spring "B" & 12 & $7 / 75$ & 65 & c & 119 & 66 & c & 139 & 137 & & $\begin{array}{r}100-130 \\
-\end{array}$ & $1 E$ & 757 \\
\hline Splashland Hot Well & 35 & & 22 & a & 35 & 23 & a & 221 & 197 & & $40-100$ & -- & 311 \\
\hline$\frac{\text { Steamboat Springs }}{\text { Heart Spring }}$ & 4 & $4 / 76$ & 101 & $q$ & 179 & 81 & $q$ & 148 & 141 & & $125-130$ & 140 & 903 \\
\hline Sulphur Cave & 4 & $4 / 76$ & 60 & $q$ & 79 & 79 & $q$ & 181 & 188 & & $125-130$ & 10 & 4,530 \\
\hline Steamboat Spring & 4 & $4 / 76$ & 66 & $q$ & .93 & 76 & $q$ & 176 & 187 & & $\begin{array}{r}125-130 \\
-\quad---\end{array}$ & $-\quad 20$ & 6,170 \\
\hline Stinking Springs & 38 & $9 / 75$ & 39 & c & 59 & 61 & c & 339 & $\begin{array}{l}41 \\
- \\
-\end{array}$ & & $\begin{aligned} & 40-60 \\
- & - \\
- & -\end{aligned}$ & $--^{24}$ & 899 \\
\hline$\frac{\text { Swissvale Warm Spgs. }}{\text { Spring "A" }}$ & 25 & $6 / 76$ & 32 & $\mathrm{cr}$ & 35 & 22 & $\mathrm{cr}$ & 214 & 48 & & $35-50$ & 125 & -- \\
\hline Spring "F" & 25 & $0 / 70$ & 31 & $\mathrm{cr}$ & 47 & 69 & $\mathrm{cr}$ & -2 & 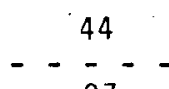 & & $\begin{aligned} & 35-50 \\
- & ---\end{aligned}$ & $\begin{array}{r}20 \\
---\end{array}$ & -- \\
\hline Trimble H.S. & 56 & $9 / 75$ & $-\cdot$ & & 34 & 47 & a & 197 & $\begin{array}{r}97 \\
---\end{array}$ & & $\begin{array}{r}45-70 \\
-\quad----.\end{array}$ & $-\ldots-$ & 3,340 \\
\hline Iripp H.S. & & $9 / 75$ & -- & & 30 & 39 & $\mathrm{a}$ & 198 & $\begin{array}{c}99 \\
--\end{array}$ & & $\begin{array}{r}45-70 \\
----.\end{array}$ & --- & 3,240 \\
\hline$\frac{\text { Valley View Hot Spgs }}{\text { Spring "A" }}$ & $-\dot{-}_{32}$ & $\begin{array}{r}6 / 75 \\
10 / 75 \\
1 / 76 \\
4 / 76\end{array}$ & $\begin{array}{l}34 \\
32 \\
32 \\
32\end{array}$ & $\begin{array}{l}c \\
c \\
c \\
c\end{array}$ & $\begin{array}{l}37 \\
35 \\
35 \\
35\end{array}$ & $\begin{array}{l}4 \\
9 \\
5 \\
9\end{array}$ & $\begin{array}{l}c \\
c \\
c \\
c\end{array}$ & $\begin{array}{l}356 \\
356 \\
352 \\
375\end{array}$ & $\begin{array}{l}12 \\
14 \\
15 \\
15\end{array}$ & & $40-50$ & $\begin{array}{l}-- \\
60 E \\
-- \\
--\end{array}$ & $\begin{array}{l}252 \\
249 \\
243 \\
234\end{array}$ \\
\hline Spring "B" & 32 & $6 / 75$ & 30 & c & 3.1 & 12 & c & 338 & 11 & & $40-50$ & -- & 234 \\
\hline Spring "D" & 32 & $\begin{array}{r}10 / 75 \\
1 / 76 \\
4 / 76\end{array}$ & $\begin{array}{l}25 \\
28 \\
28\end{array}$ & $\begin{array}{l}\mathrm{c} \\
\mathrm{c} \\
\mathrm{c}\end{array}$ & $\begin{array}{l}29 \\
31 \\
31\end{array}$ & $\begin{array}{l}33 \\
25 \\
29\end{array}$ & $\begin{array}{l}c \\
c \\
c\end{array}$ & $\begin{array}{c}360 \\
346 \\
389 \\
-\end{array}$ & $\begin{array}{r}11 \\
16 \\
10 \\
--\end{array}$ & & $40-50$ & $\begin{array}{r}120 \mathrm{E} \\
75 \mathrm{E} \\
75 \mathrm{E} \\
----\end{array}$ & $\begin{array}{l}229 \\
247 \\
223\end{array}$ \\
\hline$\frac{\text { Wagon Wheel Gap }}{\text { 4UR Spring }}$ & 43 & $\begin{array}{r}10 / 75 \\
1 / 76 \\
4 / 76\end{array}$ & $\begin{array}{l}75 \\
81 \\
77\end{array}$ & $\begin{array}{l}c r \\
c r \\
c r\end{array}$ & $\begin{array}{l}113 \\
137 \\
119\end{array}$ & $\begin{array}{l}56 \\
66 \\
59\end{array}$ & $\begin{array}{l}\mathrm{cr} \\
\mathrm{cr} \\
\mathrm{cr}\end{array}$ & $\begin{array}{l}206 \\
204 \\
200\end{array}$ & $\begin{array}{l}194 \\
191 \\
188\end{array}$ & & -- & $\begin{array}{l}30 E \\
30 E \\
28 E\end{array}$ & $\begin{array}{l}1,580 \\
1,550 \\
1,620\end{array}$ \\
\hline CF \& I Spring & 43 & $\begin{array}{r}8 / 75 \\
10 / 75 \\
1 / 76 \\
4 / 76\end{array}$ & $\begin{array}{l}71 \\
66 \\
80 \\
66\end{array}$ & $\begin{array}{l}c r \\
c r \\
c r \\
c r\end{array}$ & $\begin{array}{r}117 \\
99 \\
157 \\
99\end{array}$ & $\begin{array}{l}64 \\
56 \\
76 \\
57\end{array}$ & $\begin{array}{l}c r \\
c r \\
c r \\
c r\end{array}$ & $\begin{array}{c}205 \\
203 \\
203 \\
206 \\
--\end{array}$ & $\begin{array}{r}181 \\
184 \\
175 \\
181 \\
-\quad-\end{array}$ & & - - - - & $\begin{array}{r}30 \\
50 \\
30 \\
32 \\
-\quad-\quad-\end{array}$ & $\begin{array}{l}1,510 \\
1,520 \\
1,540 \\
1,470\end{array}$ \\
\hline$\frac{\text { Waunita Hot Springs }}{\text { Spring "C" }}$ & 46 & $\begin{array}{r}7 / 75 \\
10 / 75 \\
1 / 76 \\
4 / 76\end{array}$ & $\begin{array}{l}143 \\
143 \\
157 \\
148\end{array}$ & $\begin{array}{l}q \\
q \\
q \\
q\end{array}$ & $\begin{array}{l}213 \\
209 \\
247 \\
225\end{array}$ & $\begin{array}{l}66 \\
64 \\
71 \\
68\end{array}$ & $\begin{array}{l}q \\
q \\
q \\
q\end{array}$ & $\begin{array}{l}179 \\
176 \\
174 \\
178\end{array}$ & $\begin{array}{l}163 \\
166 \\
159 \\
167\end{array}$ & & $175-225$ & $\begin{array}{l}-- \\
30 \\
55 \\
50\end{array}$ & $\begin{array}{l}557 \\
579 \\
613 \\
575\end{array}$ \\
\hline Spring "D" & 46 & $7 / 75$ & 153 & $q$ & 291 & 83 & $q$ & 175 & 165 & & $175-225$ & -- & 594 \\
\hline
\end{tabular}


TABLE 2 CONT.

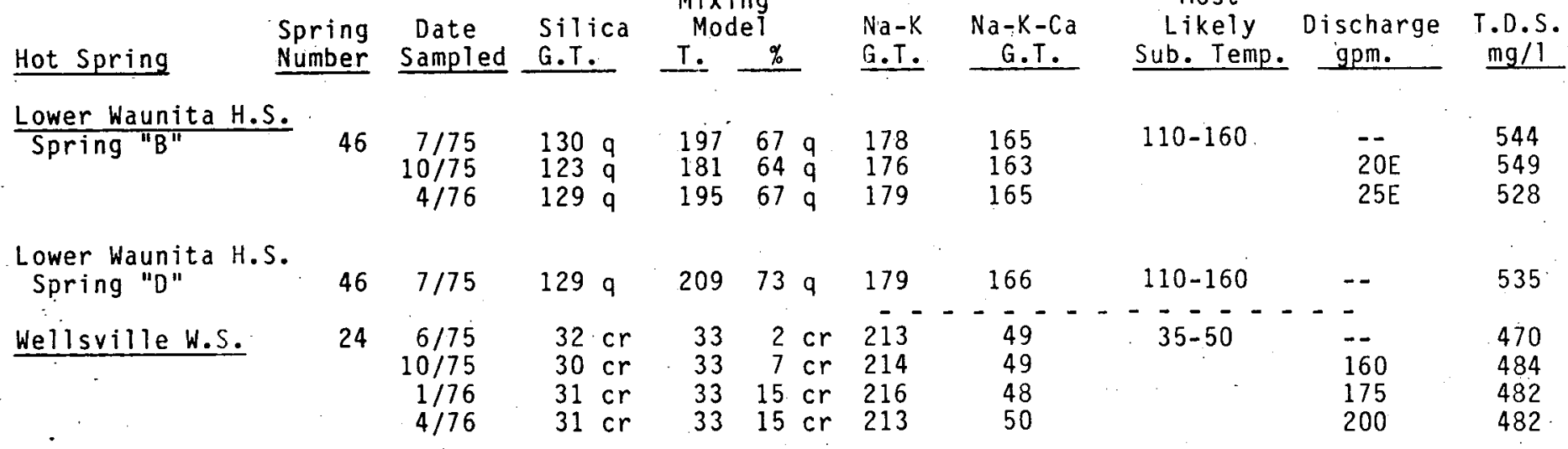


TABLE 3

Resource Assessment

of

Hydrothermal Resources in Colorado

adapted from Pearl (1979)

Thermal

Spring Areas

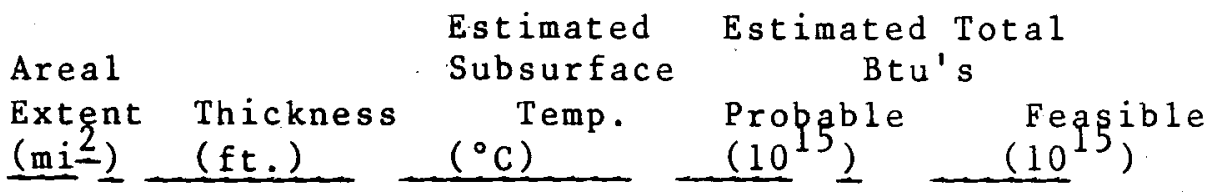

\begin{tabular}{|c|c|c|c|c|c|c|}
\hline Juniper & 1 & 1.00 & $200(\mathrm{~S})$ & 63 & .0163 & .0163 \\
\hline \multirow[t]{2}{*}{ Craig } & 2 & $\quad 1.00$ & $500(\mathrm{U})$ & 55 & .0329 & -- \\
\hline & & 12.0 & $500(\mathrm{U})$ & 55 & -- & .395 \\
\hline \multirow[t]{2}{*}{ Routt } & 3 & 0.5 & $1000(\mathrm{~F})$ & 138 & .1110 & .1110 \\
\hline & & 0.75 & $1000(\mathrm{~F})$ & 138 & .1663 & .1663 \\
\hline Steamboat & 4 & 0.52 & $1000(\mathrm{~F})$ & 70 & .0487 & .0487 \\
\hline \multirow[t]{2}{*}{ Brand's Ranch } & 5 & 0.36 & $200(\mathrm{~s})$ & 49 & .0039 & -- \\
\hline & & 1.5 & $200(\mathrm{~s})$ & 49 & -- & .0164 \\
\hline Hot Sulphur & 6 & 1.35 & $500(\mathrm{U})$ & 75 & .0698 & .0698 \\
\hline \multirow[t]{2}{*}{ Haystack Butte } & 7 & 0.54 & $300(\mathrm{~s})$ & 40 & .0061 & -- \\
\hline & & 1.50 & $300(\mathrm{~s})$ & 40 & -- & .0169 \\
\hline E1dorado & 8 & 0.52 & $1000(\mathrm{~F})$ & 35 & .0147 & .0147 \\
\hline Idaho & 9 & 1.52 & $1000(\mathrm{~F})$ & 80 & .1714 & .1714 \\
\hline Dotsero & 10 & 0.50 & $250(\mathrm{~S})$ & 39 & .0045 & .0045 \\
\hline \multirow[t]{2}{*}{ Glenwood } & 11 & 1.32 & $250(\mathrm{~S})$ & 65 & .0279 & .0279 \\
\hline & & 0.50 & $250(\mathrm{~s})$ & 65 & $(.0106)$ & $(.0106)$ \\
\hline South Canyon & 12 & 0.1 & $200(\mathrm{~s})$ & 75 & .0021 & .0021 \\
\hline \multirow[t]{2}{*}{ Penny } & 13 & 1.61 & $1000(\mathrm{~F})$ & 75 & .1664 & -- \\
\hline & & 4.7 & $1000(\mathrm{~F})$ & 75 & -- & .486 \\
\hline Colonel Chinn & 14 & 1.55 & $200(\mathrm{~S})$ & 51 & .0181 & .0181 \\
\hline \multirow{3}{*}{ Cement Creek } & 15 & 0.45 & $200(\mathrm{~S})$ & 45 & .0042 & .0042 \\
\hline & 16 & 0.28 & $1000(F)$ & 45 & .0132 & -- \\
\hline & & 1.40 & $1000(\mathrm{~F})$ & 45 & -- & .0658 \\
\hline \multirow[t]{2}{*}{ Ranger } & 17 & 0.30 & $150(\mathrm{~S})$ & 45 & .0021 & -- \\
\hline & & 0.88 & $150(\mathrm{~s})$ & 45 & -- & .0062 \\
\hline \multirow[t]{2}{*}{ Rhodes } & 18 & 1.53 & $1000(\mathrm{~F})$ & 35 & .0432 & -- \\
\hline & & 7.1 & $1000(\mathrm{~F})$ & 35 & -- & .2003 \\
\hline Hartse 1 & 19 & 1.00 & $500(U)$ & 70 & .0470 & .0470 \\
\hline \multirow[t]{2}{*}{ Cottonwood $\mathrm{Cr}$} & eek 20 & 1.38 & $1000(\mathrm{~F})$ & 170 & .3894 & -- \\
\hline & & 4.14 & $1000(\mathrm{~F})$ & 170 & -- & 1.1672 \\
\hline \multirow[t]{3}{*}{ Mt. Princeton } & $2 \cdot 1$ & 3.14 & $1000(\mathrm{~F})$ & 200 & 1.0623 & -- \\
\hline & & 4.0 & $1000(\mathrm{~F})$ & 200 & -- & $(1.3544)$ \\
\hline & & 10.0 & $1000(\mathrm{~s})$ & 160 & -- & 2.6336 \\
\hline \multirow[t]{2}{*}{ Browns Canyon } & 22 & 1.50 & $1000(\mathrm{~F})$ & 100 & .2256 & -- \\
\hline & & 3.23 & $1000(\mathrm{~F})$ & 100 & -- & .4856 \\
\hline \multirow[t]{2}{*}{ Poncha } & 23 & 0.60 & $1000(\mathrm{~F})$ & 145 & .1410 & -- \\
\hline & & 5.07 & $1000(\mathrm{~F})$ & 145 & -- & 1.1911 \\
\hline
\end{tabular}


TABLE 3 (Cont.)

Therma 1

Spring Areas
Estimated

Areal

Extent Thickness (mi2) (ft.)
Subsurface

Temp. $\left({ }^{\circ} \mathrm{C}\right)$
Estimated Total

Probable

Feasiple

0028

.0028

.0095

.0083

$-$

.0353

.0083

.9492

$--$

.0564

.0148

.1551

.1551

.0339

.0257

$--$

.0169

.0226

.0470

$--$

.0625

$500(\mathrm{U})$

$500(\mathrm{U})$

$500(\mathrm{U})$

$500(\mathrm{U})$

$200(\mathrm{~S})$

$500(\mathrm{~s})$

$500(\mathrm{~S})$

$1000(\mathrm{~F})$

$425(S \& F)$

$400(S \& F)$

1.20

Lemon

49
50

2.53

2.00

0.80

Dunton/Geyser/Paradise $51 / 52 / 53$

$\begin{array}{lll}\text { Dunton } & 51 & 0.30 \\ \text { Geyser } & 52 & 0.30 \\ \text { Paradise } & 53 & 1.00 \\ \text { Rico } & 54 & 2.15 \\ \text { Pinkerton/Mound } 55 & 0.98 \\ & & 2.06 \\ \text { Tripp/Trimble } 56 & 1.0\end{array}$

Total

(S) Stratigraphic reservoir

(F) Fracture reservoir

(U) Unknown
$400(S \& F)$

$400(S \& F)$

$400(\mathrm{~S} \& \mathrm{~F})$

$1000(\mathrm{~F})$

$180(S \& F)$

$180(S \& F)$
$500(F \& S)$
.0113

$--$

.0606

.0284

- -

.2256

.0149

--

.0068

.0068

.0226

.1738

.0099

--

.0357

4.8782
.0095

--

.0430

--

$-$

$-$

3.007

.0564

.0148

.1551

.1551

.0339

-

.0620

.0169

.0226

--

.0941

--

1.4285

-

.0880

.0606

--

.1308

.2256

.0149

.0271

-

--

$-$

.1738

--

.0209

.0357

13.2386 
TABLE 4

FEDERAL ACTIVE COMPETITIVE GEOTHERMAL LEASES

(KGRA'S)*
IN COLORADO
December, 1977

LESSEE

ACRES TOWNSHIP \& RANGE DATE ISSUED

The Anschutz Corporation

915

$49 \mathrm{~N}, 8 \mathrm{E}$

1975

Phillips Petroleum Company

2484

$45 \mathrm{~N}, 8 \mathrm{E}$

1975

Phillips Petroleum Company

1636

$45 \mathrm{~N}, 9 . \mathrm{E}$

1975

5035

*Designated Known Geothermal Resource Areas by Federal Government SOURCE: Bureau of Land Management, Denver, Colorado 
TABLE $\%$

FEDERAL ACTIVE NON-COMPETITIVE GEOTHERMAL LEASES

IN COLORADO

December, 1977

\section{LESSEE}

Buttes Resources Company

Chevron. Oil Company

Earth Power Corporation

Geothermal Kinetics, Inc.

Ladd Petroleum Corp.

Occidental Petroleum

Phillips Petroleum Co.

\section{ACRES}

781.32

$2,226.88$

$1,804.57$

$1,040.04$

$1,970.30$

$1,867.94$

$2,127.56$

645.74

160.00

$1,000.00$

$1,000.00$

$1,106.00$ 827.31

$1,042.47$

383.65 280.00

80.00

$1,280.00$

$2,113.30$

$1,549.66$

$1,286.17$

$\begin{array}{r}320.00 \\ 1,120.00 \\ 1,644.50 \\ 329.50 \\ \hline\end{array}$

$28,487,51$
TOWNSHIP \& RANGE

$46 \mathrm{~N}, 2 \mathrm{~W}$

$46 \mathrm{~N}, 1$ \& $2 . \mathrm{W}$

$46 \mathrm{~N}, 1 \mathrm{~W}$

$46 \& 47 \mathrm{~N}, 2 \mathrm{~W}$

$46 \& 47 \mathrm{~N}, 2 \mathrm{~W}$

$46 \& 47 \mathrm{~N}, 2 \& 3 \mathrm{~W}$

$46 \& 47 \mathrm{~N}, 3 \mathrm{~W}$

$47 \mathrm{~N}, 3 \mathrm{~W}$

$45 \mathrm{~N}, 9 \& 10 \mathrm{E}$

$46 \mathrm{~N}, 10 \mathrm{E}$

$37 \mathrm{~N}, 12 \mathrm{E}$ \&

$38 \mathrm{~N}, 13 \mathrm{E}$

$29 \mathrm{~W}, 73 \mathrm{~W}$

38. $\mathrm{N}, 13 \mathrm{E} \&$

$29 \mathrm{~S}, 73 \mathrm{~W}$

$29 \mathrm{~S}, 73 \mathrm{~W}$

$45 \mathrm{~N}, 11 \mathrm{E}$

$46 \mathrm{~N}, 10 \mathrm{E}$

$49 \mathrm{~N}, 8 \mathrm{E}$

$49 \mathrm{~N}, 3 \mathrm{E}$

$49 \mathrm{~N}, 7 \& 8 \mathrm{E}$

$49 \mathrm{~N}, 9 \mathrm{E}$

$51 \mathrm{~N}, 8 \mathrm{E}$

$46 \mathrm{~N}, 10 \mathrm{E}$

$45 \mathrm{~N}, 10 \mathrm{E}$

$46 \mathrm{~N}, 10 \mathrm{E}$

$46 \mathrm{~N}, 11 \mathrm{E}$
DATE ISSUED

1977

1977

1977

1977

1977

1977

1977

1977

1975

1976

1975

1975

1975

1975

1975

1975

1975

1975

1975

1975

1975

1975

1975

1975

1975

SOURCE: Bureau of Land Management, Denver, Colorado 
TABLE

COLORADO STATE ACTIVE GEOTHERMAL LEASE

December, 1977

LESSEE

General Geothermal, Inc.

Geothermal Products, Inc.

Mapco, Inc.

Marine Minerals

Occidental Oil Co.

Petro-Lewis

Phillips Petroleum

Underwood, C. A.

TOTAL

$\begin{array}{ll}\text { ACRES } & \text { TOWNSHIP \& RANGE } \\ 9,639.90 & 41 \mathrm{~N}, 6 \mathrm{E} \\ & 45 \mathrm{~N}, 9 \mathrm{E} \\ & 46 \mathrm{~N}, 10 \mathrm{E} \\ 8,183.33 & 39 \mathrm{~N}, 11 \mathrm{E} \\ & 40 \mathrm{~N}, 12 \mathrm{E} \\ 360.00 & 49 \mathrm{~N}, 8 \mathrm{E} \\ 640.00 & 35 \mathrm{~N}, 2 \mathrm{~W} \\ 640.00 & 12 \mathrm{~S}, 75 \mathrm{~W} \\ 480.00 & 11,12 \mathrm{~S}, 75,76 \mathrm{~W} \\ 560.00 & 35 \mathrm{~N}, 1 \mathrm{~W}\end{array}$

$\begin{array}{llll}2,004.85 & 14 & \mathrm{~S}, 78 \mathrm{~W} \\ 3,692.00 & 15 & \mathrm{~S}, 78 \mathrm{~W} \\ 1,280.00 & 15 \mathrm{~S}, 78 \mathrm{~W} \\ & 14 \mathrm{~S}, 78 \mathrm{~W}\end{array}$

$640.0040 \mathrm{~N}, 11 \mathrm{E}$

$1,625.00 \quad 41 \mathrm{~N}, 11,12 \mathrm{E}$

$17,895.00$

$9,611.88$

$3,920.00$

$41 \mathrm{~N}, 10,11,12 \mathrm{E}$

$39 ; 40 \mathrm{~N}, 11,12 \mathrm{E}$

$37,38 \mathrm{~N}, 11,12 \mathrm{E}$

$2,532.24$

$42,43,44,45 \mathrm{~N}$,

$10 \mathrm{E}$

$\begin{array}{rll}640.00 & 40 \mathrm{~N}, 12 \mathrm{E} \\ 640.00 & 49 \mathrm{~N}, 4 \mathrm{E} & \\ & & \\ 2,004.85 & 14 \mathrm{~S}, 78 \mathrm{~W} & \\ 3,692.31 & 15 \mathrm{~S}, 78 \mathrm{~W} & \\ 1,280.00 & 15 \mathrm{~S}, 78 \mathrm{~W} & \\ & 14 \mathrm{~S}, 79 \mathrm{~W} & \\ 3,226.61 & 50 \mathrm{~N}, 8 \mathrm{E} & \\ 1,560.00 & 49 \mathrm{~N}, 7 \mathrm{E}, 9 \mathrm{E} \\ & 50 \mathrm{~N}, 8 \mathrm{E} & \\ 40.00 & 14 \mathrm{~S}, 78 \mathrm{~W} & \end{array}$

$1,764.40$

$48,49 \mathrm{~N}, 4,5 \mathrm{E}$

$3,520.00$

$14 \mathrm{~S}, 78 \mathrm{~W}$

$45,46 \mathrm{~N}, 8,9,10 \mathrm{E}$

$1,120.00 \quad 33 \mathrm{~N}, 8 \mathrm{E}$
COUNTY

Saguache

Alamosa

Chaffee

Archuleta

Park

Archuleta

Chaffee

Al amos a

Saguache

Al amos a

Saguache

Al amosa

Gunnison

Chaffee

Gunnison \&

Saguache

Chaffee

Conejos, \&

Saguache

Conejos 
TABLE 7

CURRENT USES OF GEOTHERMAL RESOURCES

IN COLORADO

1978

Type of Use

Swimming Pools

Baths
Name of Area

Juniper Hot Springs

Steamboat Hot Springs

Hot Sulphur Springs

Eldorado Warm Springs

Idaho Hot Springs

Glenwood Hot Springs

Cement Creek Hot Springs

Cottonwood Creek Hot Springs

Mt. Princeton Hot Springs

Hortense Hot Springs

Poncha Hot Springs

Shaws Warm Springs

Splashland Hot Water We 11

Pagosa Hot Springs

Wagon Wheel Gap Hot Springs

Upper Wuanita Hot Springs

Ouray Hot Springs

Pinkerton Hot Springs

Valley View Hot Springs

Juniper Hot Springs

Hot Sulphur springs

Idaho Hot Springs

Glenwood Hot Springs

South Canyon Hot Springs

Mineral Hot Springs

Valley View Hot Springs

Cebolla Hot Springs

Orvis Hot Springs

Ouray Hot Springs

Dunton Hot Springs

Paradise Hot Springs 
TABLE 7 (Cont.)

Space Heating

Other

Laundry

Greenhouses

Algae Growing

Irrigation

Bottled Water

Fish Farming
Cottonwood Creek/Jump Steady Hot Springs -

Mt. Princeton - cabins/House, resort

Wright Hot Water We1ls - 2 houses

Poncha Hot Springs - 1 house

Sand Dunes Swimming Pool Well - 1 house

Ouray - 2 motels

Pagosa Springs - approx. 10 buildings

Upper Waunita Hot Springs - headquarters bldg.
Hot Sulphur Springs

Penny Hot Springs

Wright Hot Water Wells

Tripp Hot Springs

Wellsville

Dutch Crowley

Clark Artesian We 11

E1 Dorado Warm Spring

Sand Dunes Hot Water We 11

SOURCES: Barrett and Pear1, 1978 and unpublished data from Dick and Galloway, 1978. 
SOME POTENTIAL USES FOR GEOTHERMAL RESOURCES IN COLORADO

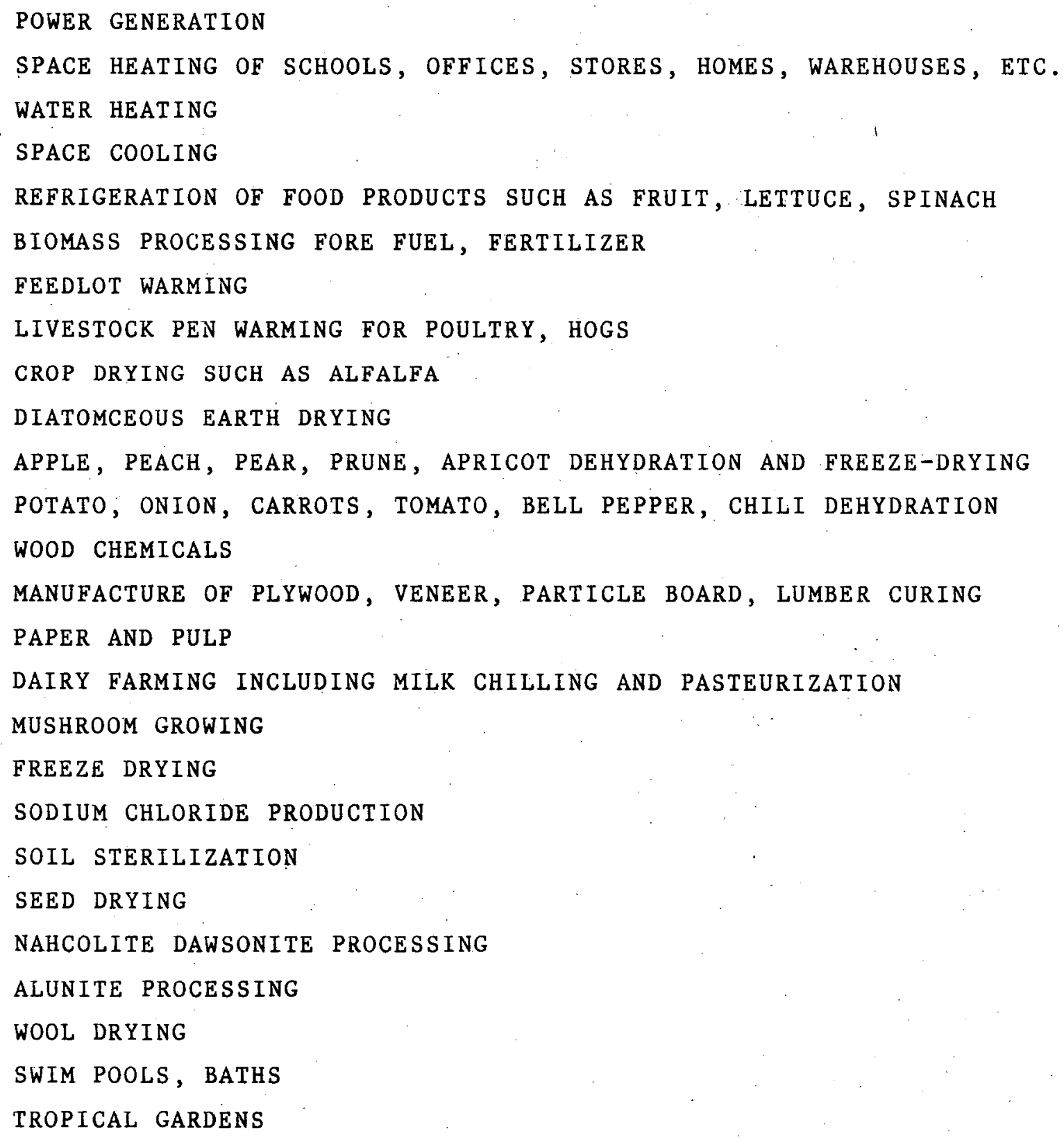


focus of this year's effort is upon the refinement of those scenarios. However, the production of one area development plan was stipulated in the work program.

\subsubsection{State Geothermal Planning Areas}

The area for which a development plan was proposed and completed is the San Luis Valley Region, Planning and Management Region 8 of the 13 regions designated by the State. As shown on the map, Figure 2, it includes the six counties of Alamosa, Conejos, Costilla, Mineral, Saguache, and Rio Grande that are located in south-central colorado. The area is described further in the plan.

\subsubsection{Area Development P1an}

The area development plan for the San Luis Valley Region relied primarily upon data obtained from the area itself, both in the form of published documents and conversations with local officials and residents. Because of this "grass roots" approach, it is considered to reflect closely the actual conditions in the Valley that will determine the ultimate extent of development. The plan is found in Appendix A. 


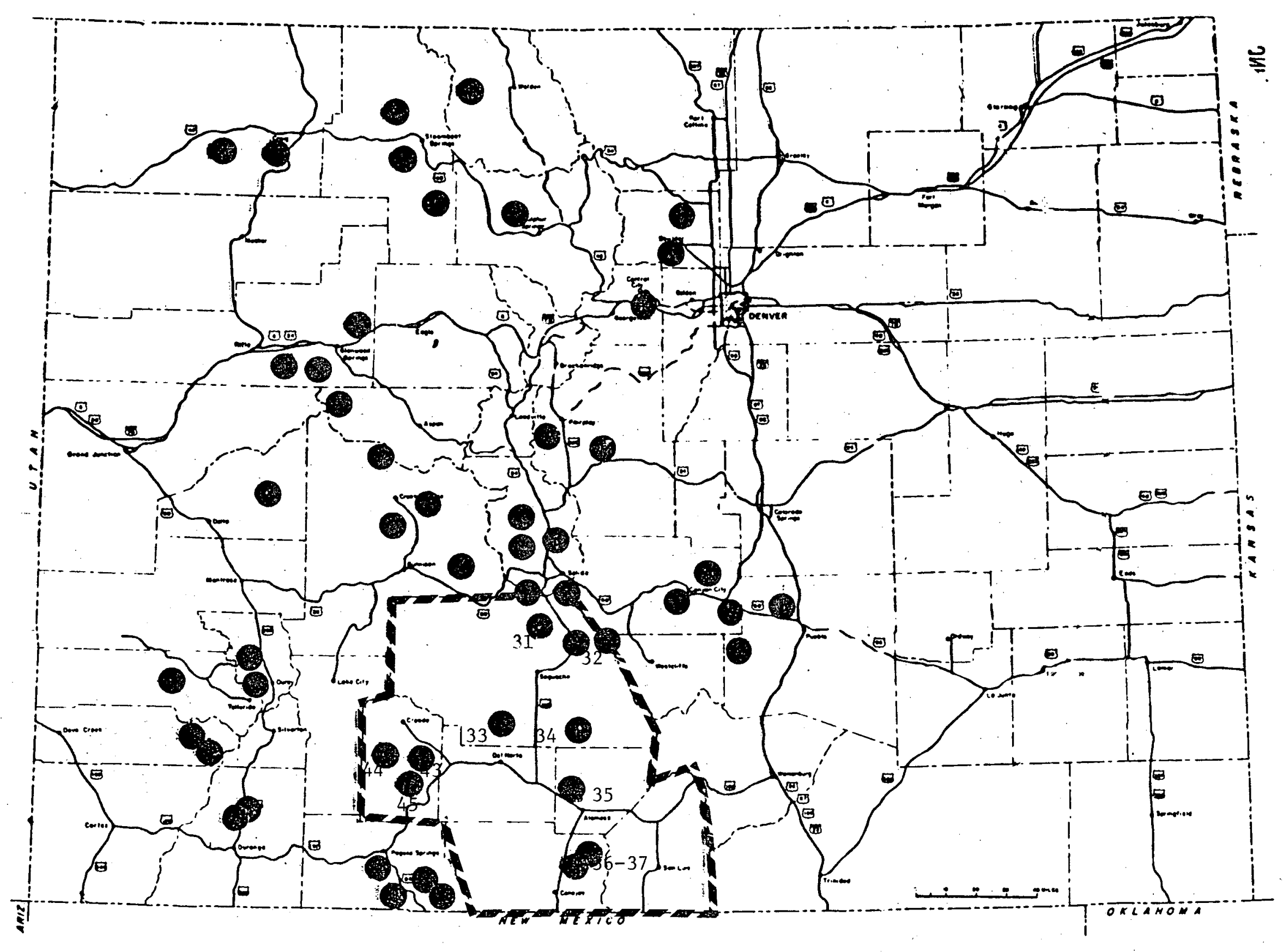

FIGURE 2

LOCATION OF COLORADO REGION 8 


\section{3 Site Specific Development Plans}

\subsubsection{Candidate Geothermal Sites/Applications}

During the last contract year, scenarios were prepared for a11 hydrothermal sites that had been identified in Colorado. These are found in Appendix B. As detailed data are obtained, the scenarios are being revised and expanded, beginning with the sites that seem to be approaching development most rapidly.

It should be especial1y noted that the original resource development categories on Table I of that report are no longer valid, with the exception of IIA and IIIC, excluding Dotsero which is being considered for highway snow-melting.

\subsubsection{Site Specific Development Plans in Preparation}

Site specific scenarios are currently being updated for four sites, Glenwood Springs, Idaho Springs, Ouray and Pinkerton. These sites were chosen because some initial development activities, beginning with, preliminary planning investigations, and attempts to obtain funds and/or technical assistance have begun. Data are now being collected for the expansion and refinement of these sites. The next version will include an analysis of the current and forecast conditions at the sites that limit or encourage development, as well as analyses of the requirements for development at each site. Based upon these investigations, possible industrial; commercial, and residential energy demand will be assessed. The Pagosa Springs and San Luis Valley scenarios have been revised. Of the remainder, those with some development activity will be considered for expansion during subsequent planning phases. 


\subsubsection{Sites with Development Activity}

As part of the investigation of geothermal activity and potential in Colorado, as well as the outreach program, new information about geothermal sites is obtained continuously. Especially relevant to the site analyses is the information about current and proposed development. The extent of interest in geothermal resource use has been surprising. Were adequate incentives available, many areas would probably be developed. The following list describes some of the geothermal resource areas of the State where development is contemplated or underway.

\#3 Routt Hot Springs - the feasibility of heating a private boarding school is being preliminarily investigated from existing data. The school may apply for a DOE "schools and hospitals" grant. Early studies show a potential to supply nearly all the heat required by Steamboat Springs with energy from Routt and steamboat Springs (Andrews, pers. comm.).

\#1 7 Haystack Butte - Landowners interested in developing greenhouses for lease to operators may apply for a guaranteed loan. A grant proposal was submitted to the "Appropriate Technology, Small Grants Program" to obtain funds to prepare an economic and engineering feasibility study. Its location near the Denver/Boulder area should enhance the market potential of greenhouse products. If grants or loans are not received, financial requirements may limit the establishment of the feasibility and subsequent development. The analysis showed a potential for heating over 300 homes with an equal amount of energy for commercial use (Vranesh, pers. comm.).

非 Eldorado Warm Springs - The geothermal wells here are already contributing to the energy needs of the lodge, swimming pool, and homes in the area by providing preheated water to boilers supplying hot water. The owner is interested in additional use of the energy to heat the lodge and new homes in the immediate vicinity. Early estimates indicated enough heat for over 100 homes. Because the water may not be warm enough, heat pumps or a solar-assisted system are suggested. The owner is interested in obtaining a grant (Fowler, pers. comm.).

\#9 Idaho Hot Springs - The owners of this hydrotherapy resort are interested in 
heating the resort building with geothermal energy. They requested information and technical assistance about grant programs. They indicated they. will drill a well and install a heating system without obtaining additional professional assistance. Wells previously supplying hot water for baths and a swimming pool have clogged, requiring the drilling of subsequent wells. Estimates have shown a reservoir potential for heating more than all the existing structures in the terms of Idaho Springs (Maxwel1, pers. comm:).

\#10 Dotsero Hot Springs - The feasibility of using natural hot water to melt snow and ice from highway structures through the sheer-walled Glenwood Canyon is being explored by the State Department of Highways. They have requested assistance from EG\&G. If it is economically feasible, lack of funds should not be a problem (Swanson, pers. comm.).

\#1l Glenwood Springs - Glenwood Springs residents and officials are seeking ways to develop their geothermal energy to heat residences, commercial buildings, and public buildings, especially a planned office building, a 22-unit condominium development, and municipal complex. The ownership of geothermal rights is a question of some concern, along with the questions of where to obtain the necessary funds for drilling a well and how to design a system to use their highly mineralized water (Schenk, West, Ross, and others, pers. comm.).

\#13 A Penny Hot Springs landowner who is a retired physician hopes to develop the geothermal waters for a clinic incorporating hydrotherapy and for a greenhouse. No problems are known yet (Johnson, pers. comm.).

\#16 Cement Creek and Ranger Warm Springs - Subdivision builders building near these areas are interested in the energy potential. They would need grant or energy developer assistance to develop geothermal energy to replace the electric resistance space heat in the homes (Mason and Bernal, pers. comm.).

\#19 Hartsel - a developer inquired about the possibility of using geothermal energy for heating a proposed resort. Soil mercury surveys throughout the area were run by the CGS during 1979 (Pear1, pers. comm.).

非23 Poncha Springs..- some interest has been expressed in this area for greenhouses, mushroom growing (Dick, pers. comm.). 
\#26 Canon City - The CGS has entered into a cooperative agreement with the Colorado Department of Institutions to try to develop geothermal energy for heating of the new prison and at the prison industries, including laundry, swine pens, feedlots, meat packing plant, and dairy. Soil mercury and geophysical tests are being performed.

\#31 Mineral Hot Springs - as shown in the San Luis Valley analysis, Mineral Hot Springs is being developed for swine, methane.

\#34 Sand Dunes - interest in heat for composting for mushroom growing. (Goering, pers. comm.).

\#35 Splashland - Superintendent of Schools interested in heating new schools. Soil mercury and geophysics are being conducted in the San Luis Valley, the same valley in which the above three sites are located, although distant from them.

\#4 1 Pagosa Springs - district heating system is planned; prospective developers have inquired about greenhouse and aquaculture potential. (Ebeling and Hays, pers. comm.).

非46 Waunita Hot Springs - county has inquired about potential for providing energy for new town (Johnson, pers. comm.).

\#49 Orvis Hot Springs - hydrotherapy facility operator has expressed a desire to develop expanded facility and industrial park (Schacter, pers. comm.).

非50 Ouray Hot Springs - widespread interest in geothermal heat for public, commercial and residential buildings, as well as for encouragement of industrial facilities to locate in Ouray. Community relies upon high-cost propane and electricity for heat and has lost their major employer with the closing of the gold and silver mine (Massard, pers. comm.).

非54 Rico - Manager of Rico Argentine Mine and Mill has attempted in the past to develop the energy to heat company houses. Currently, an option is held on the company and mining operations have closed temporarily. The possibility of development awaits a decision concerning that option (Jahnke, pers. comm.). 
\#55 Pinkerton - Private boarding schoo1. is attempting to develop the energy for space heat, because high-cost propane is prohibitive to the school. A grant application was submitted to DOE's "Sma11 Grants" program to construct a small heating system. However, when the Colorado Division of Highways constructed a new section of Federal highway, the conduit of the spring that formerly flowed into a pit on the school property was severed. Since it began discharging onto the land, several huge pine trees have withered. The Highway Division was investigating ways to prevent the discharge of the fluid onto school property, because of their liabiity for environmental damage. They may also be liable for restoring the energy resource to the original owners. (Allen, pers. comm.).

\subsection{Time Phased Project Plans}

\subsubsection{Active Demonstration/Commercialization Projects}

Only two sites in Colorado are currently being developed. One is the Mineral Hot Springs site in the San Luis Valley. As indicated in the San Luis Valley plan (Appendix A), Weisbart's, Inc., is constructing a geothermally heated swine pen for 30,000 head, as well as a methane plant to produce methane for operating a power generator (Weisbart, Gary, pers. comm.). About $7.9 \times 1010$ Btu's of geothermal energy is expected to be on line at Mineral Hot Springs by the year 1980 .

The other project is the Pagosa Hot springs project. Funds were obtained from DOE to help finance a district heating. system to heat about 60 buildings in the Pagosa Springs central business district. Approximately $5 \times 1010$ Btu's of geothermal energy are expected to be on line by 1981 , including the existing development in Pagosa Springs.

\subsubsection{Time Phased Project Plans - Completed}

The Time Phased Project Plan for Pagosa Springs is found in Appendix C. 


\subsection{State Aggregates of Prospective Geothermal Utilization}

Following is the amount of geothermal energy that is preliminarily estimated to be developable in Colorado by the year 2020. These estimates will be revised in the near future.

Electric Power Generation Sites

Site

Potential Energy on Line by the Year 2020

Chalk Creek $21 \mathrm{~A}$

$100 \mathrm{MWe}$

Poncha Springs 23

200 MWe

Cebolla 47

$200 \mathrm{MWe}$

$500 \mathrm{MWe}$

Direct Thermal Sites or Areas

San Luis Va1ley 非1/32/33/34/35/36-37/43/44/45

1010 Btu's

*Pagosa Springs 非 1

422

189

Glenwood Springs 非1

129.

'Hartsel 非19

4. 0

Waunita $\$$ \$ 6

12 .

Routt/Steamboat \#3,4

25.

Hot Sulphur \#6

14.

Haystack Butte 非 7

3.

E1dorado $\$ 8$

2.

Idaho 非 9

8.

Ouray 非 49

15.

Dunton/Geyser/Paradise 非 $51,52,53$

5.

Juniper/Craig 非1, 2

12.0

Brand's Ranch 非 5

.7

South Canyon 非 12

2.0

Penny 非13

10.0

Colonel Chinn 非14

4.0

Cement Creek/Ranger \#16,17

3.0 
We11sville/Swissvale 非24, 25

Canon City, Fremont 非 6,27

Don K. Ranch, Florence \#28,29

Clark $\# 30$

Wagon Wheel Gap 非43

Orvis \#48

Rico \#54

Pinkerton/Mound 非5

Tripp/Trimble 非 56

2.0
3.0
19.
2.0
4.0
6.0
4.0
2.0
7.0
TOTAL $\quad 909.7 \times 1010 \mathrm{Btu}$ 's




\subsubsection{Overview of State Laws and Regulations}

The following section describes the primary legislation and procedures relative to geothermal energy development in Colorado. It is excerpted from C.G.S. Information Series 9 (Coe, 1978).

Before any new geothermal development can occur in Colorado, certain legal requirements must be met. In this section, the two primary requirements, obtaining leases and permits, are discussed. As indicated, the requirements vary depending upon whether the ownership of the land and/or mineral rights is federal, state, or private.

Leasing

Developers generally have obtained geothermal leases on wide areas, usually several thousand acres, in an area in which they are interested. This assures that they will have adequate control of any discovery that might be made. In the case of federal land, control in advance of a discovery is especially vital. If a discovery is made, the area may be designated a Known Geothermal Resource Area (KGRA). In KGRA's, leasing is competitive, with no allowance for preferential leasing rights to the company making the discovery. For this reason, a company must have control of the area before they can justify spending large sums of money on exploration. In Colorado, the KGRA provision usually makes the acquistion of a combination of federal, state and private leases necessary. Especially where much of the land ownership is "checkerboarded" or split among various types of jurisdictions, obtaining several types of leases may be the only way to control sufficient amounts of acreage. The steps necessary for obtaining leases vary depending upon what entity owns the land and the minerals.

Private Lands and Minerals

Where ownership is private, leases are obtained simply through negotiation and contractual agreement with the owner. The transaction is then usually reported by the lessee to the county clerk of the county or counties in which leases are located.

State-Owned Lands or Minerals

Leasing of state-owned land and minerals is under the jurisdiction of the State Board of Land Commissioners, whose mandated objective is to maximize the income from state lands. Under the Board are four - sections, Minerals, Rights-of Way, Surface and Accounting. The Minerals Director is responsible for administration of geothermal 
leases, and the accounting department is responsible for collection of the rental and royalty payments for these leases ( $T$. E. Bretz, 1978, pers. comm.).

To obtain a state geothermal lease, an application is filed with the Minerals section. The request is then posted on the bulletin board, and a questionnaire is c1rculated for comment among state, local and federal agencies, as well as county commissioners' and county planners' offices in the respective county or counties. The feasibility and impacts of the proposal are evaluated, taking into consideration the comments returned from other agencies and individuals. After reviewing all the information, the Board decides whether or not to issue the lease. The entire process usually requires about six months. Leases may also be auctioned, but have been noncompetitive thus far because of limited interest in geothermal leasing.

Prior to any exploration or development, the Board must be notified. The applicant must also post bond to guarantee compliance with the Board's requirements for restoration of the surface and settlement of damages to the surface property. The lessees must agree to conduct his operations in a maner satisfactory to federal and state agencies concerned with air and water pollution. Further, he is required to submit reports following driling, and to pay an annual rental, a minimum royalty and, where there is production, a production royalty. The amounts are established by the Board.

Although various types of leases have been issued by the Land Board since adoption of the Colorado Constitution, geothermal leases have been issued only since 1973, when the Geothermal Resources Act was adopted (CRS 36, Art. 1, Sec. 113).

\section{Federal Lands or Minerals}

Geothermal leases on federal land or mineral ownership are issued by the Bureau of Land Management (BLM) of the U.S. Department of the Interior (USDI), as mandated by the Regulations on the Leasing of Geothermal Resources (43 CFR 3210.2). The procedures for leasing vary depending upon whether or not the area has been designated a Known Geothermal Resources Area (KGRA).

If the area is not a KGRA, an application for leases is filed with the BLM. The surface management agency, which may be the BLM, the U.S. Forest Service (USFS) or the Bureau of Indian Affairs (BIA), in consultation with the United States Geological Survey. (USGS), assesses the probable environmental impacts and determines the necessary mitigation procedures, which are added to the lease application. The Menlo Park, California, USGS office reviews the lease stipulations for adequacy and for compatibility with development of the lease. The USGS investigates to ensure that the area has not been classified as a KGRA following submittal of the application. The lease is then sent to the applicant who may accept or refuse the lease with its stipulations.

Lease applications may also be rejected by the BLM in the event the 
environmental analysis indicates that critical environmental considerations and mitigation measures cannot be implemented ( 43 CRF 3210.4 ).

The Geothermal Steam Act defines "Known Geothermal Resources Area" as "an area in which the geology, nearby discoveries, competitive interests, or other indicia would, in the opinion of the Secretary, engender a belief in men who are experienced in the subject matter that the prospects for extraction of geothermal steam or associated geothermal resources are good enough to warrant expenditures of money for that purpose" (P.L. 91-581):

Based upon this definition, all wells within a five mile area of a discovery well are classified as part of the KGRA if the extent of the geologic structure is unknown, unless the USGS determines that the geothermal resource is in a different geologic structure. If the geologic structure is known, a11. land in the structural area is considered to be part of the KGRA. In addition, if during the same filing period, two or more applications have an overlap of 50 percent or more, the entire area is classified as an administrative KGRA (Kenneth Bul1, 1978, pers. comm.).

In a KGRA, lands are leased competitively to the bidder offering the highest bonus bid, if it is above the minimum acceptable bid. Before offering such lands for lease, the impacts on the environment must be assessed, the comments of appropriate federal agencies, business and industry and private organizations must be solicited, and public hearings may be held. If issuance of leases may significantly affect the environment, an environmental impact statement may be required (Kenneth Bul1, 1978, pers. comm.).

The first term of any lease is for 10 years. The law requires diligent exploration, determined by a review of the USGS Area Geothermal Supervisor. After the fifth year, annual exploration expenditures must be at least twice the year's rental payment. However, expenditures during the first five years as well as excess expenditures during subsequent years may be credited to help fill the expenditure requirements. The lease may continue for up to 40 years as long as long as geothermal steam is produced or utilized in commercial quantities, with preferential right to renewal for a second 40-year term (43 CFR 3203.1).

In some cases the land is privately owned, while the mineral rights are owned by the federal government. After a long appraisal, the courts in 1978 determined that the geothermal resources belong with the mineral rights. This means that both mineral and surface rights must be obtained from their respective owners in order to conduct any geothermal operations on such properties.

\section{Permits}

The permit requirements for geothermal operations also vary depending upon the ownership, except that prior to drilling any geothermal well in Colorado, a permit must be obtained from the Colorado $0 i 1$ and Gas Conservation Commission. Other state permits may be required, depending upon the particular activities. In some cases, the county 
or municipality in which the well will be drilled may also require a permit. If the well will be drilled on federal or state land, the approval of the respective government agency is required.

\section{State Permits}

To obtain a permit to drill (or deepen) a geothermal well, an application must be filed with the Director of the $0 i 1$ and Gas Conservation Commission, along with a filing and service fee of $\$ 75.00$. If the permit is requested for an exploratory well, the application must include a "written statement based on competent geologic opinion or data derived from similarly situated geothermal resource areas containing whatever information the Commission requires to carry out the purposes s described in Section 34-70-102 of the Act." (State of Colorado, 0il and Gas Conservation Commission, 1975). If the permit requested is for a development well, information is required... (see following section).

An application for a geothermal well must also be referred by the Commission to the State Engineer for his determination whether the well will have any adverse effect upon existing water rights. Either he must, within 60 days, find no adverse effect, or he must recommend denial of the permit (Frank Piro, 1977, 1978, pers. comm.).

Permit for Fluid Discharge - In the case of geothermal exploration, field development or power plant operation, a developer may wish to discharge geothermal fluid. into a stream or injection well. If so, he must obtain a permit from the Colo. Dept. of Health, Water Quality Control Division...

Air Pollution Permit - The Colorado Department of Health Air Pollution Control Commission and its staff are responsible for controlling point-source emissions and ambient air quality as established by the Federal Clean Air Act and the Colorado Air Pollution Control Act of 1970 (Air Pollution Control Commission, 1977). A potential developer is advised to contact the agency even when not anticipating the release of any material into the air. If the Commission officially exempts a project, the developer will be protected in case of any unexpected release of pollutants. Furthermore, the developer will wish to be informed about all the regulations that are applicable.

Geothermal System Development Permits-Permits are required in order to construct a power plant or utility system except where a municipally owned public utility will provide service within its boundaries.

The Colorado Pub1ic Utilities Commission (PUC) issues permits and regulates activities of common carriers, pipelines, electrical facilities, telephone systems, telegraph systems, or water systems for public use.

Federa1 Permits

Geothermal operations on federal lands or minerals are under the jurisdiction of the USGS, subject to the authority of the Secretary 
of the Interior and the Director of USGS ( $30 \mathrm{CFR}, 270.10)$. Some preliminary exploration and "casual" uses that do not disturb the land, improvements or resources may be done without obtaining leases (43 CFR 3209.0).

If leases are held, approval may be obtained from the surface management agency of a "Notice of Intent and Permit to Conduct Exploration Operations", in order to conduct such activities as geochemical and geophysical surveying and driling of shallow gradient holes ( 43 CFR 3209.0).

Prior to drilling thermal gradient holes, lessees have been required to obtain approval of a plan of operations, a lengthy process. Recent regulation revision, however, requires submittal by the lessee of a description of development and its approval of the U.S.G.S. With this change, a lessee or non-lessee may now obtain approval in most cases in less than 30 days (Kenneth Bu11, 1978, pers. comm.).

Any drilling other than thermal gradient holes may be done only by the lessee or his agent on his own leases. Prior to conducting activities, a plan of operations must be approved by the USGS. Such plans of operation may cover one or a combination of activities, as follows: exploration, collection of baseline data, development, injection, production, and utilization. Al1 require an environmental analysis which is reviewed by numerous organizations and individuals (60 in Colorado, at this time) (Kenneth Bul1, 1978, pers. comm.).

The plan of operations must include, in addition to a description of the primary activities, a description of the measures to be taken for protecting the environment, for disposal and reclamtion, and for monitoring. If the plan of operations is for production, disposal or utilization plans, baseline data are required, including air and water quality, noise, seismic and land subsidence and ecological systems covering a period of one year. The baseline data may be obtained from other sources if available. After the environmental analysis is conducted by the USGS, it is reviewed by the surface management agency and must be jointly approved by the two agencies. Interested parties, including state and local governments, are invited to comment. The entire process usually requires a minimum of from three to six months. (30 CFR 270.35).

Plans of operation are reviewed by a geothermal advisory panel. If the area is a newly identified geologic area or if the operation is unusual, the panel may hold a public hearing, extending the review process to about nine months' time. A full environmental impact statement (E.I.S.) may be required for any plan if considered to be necessary by Washington offices of the responsible agencies. An EIS usually adds about two years to the process (Kenneth Bull; 1978, pers. comm.).

A geothermal operator on federal leases must file reports periodically, including:

1. Report of significant effect on the environment or industrial accident.

2. Log and well history. 
3. Monthly report of operations.

4. Monthly report of sales and royalties.

5. Annual report of compliance with environmental protection requirements.

6. Annual report of expenditures for diligent exploration operations.

Production royalties are determined by the area supervisor of the U.S.G.S. (30 CFR 270.02).

\section{Local Permits}

Both counties and municipalities are permitted by legislation to regulate numerous activities, including geothermal development, within their boundaries. But such authority must be specifically implemented by adopting regulations. Not all local governments in the state have felt it necessary or wise to expand their regulations to include geothermal resource development. In those instances where regulations for geothermal development have been adopted, they are often made a part of the subdivision regulations.

Following is a description of the procedures required by one county where geothermal resources are regulated. Since the procedures would. probably be similar in other areas, this should provide an indication of the kinds of requirements to be expected.

Alamosa County - Alamosa County has included in their subdivision regulationsmineral regulations that specifically refer to geothermal resources. To obtain county approval to develop a geothermal resource, the developer first meets with the planning commission or staff informally about the specific requirements. He then prepares and submits a proposal that is circulated to various agencies, including the Colorado Geological Survey, for review and comment. After those comments have been received, the application goes through final review by the planning commission, which recommends denial or approval. Then the proposal is submitted to the Board of County Commissioners, which holds a public hearing to allow interested parties to comment. The Commissioners then decide whether or not to allow the activity.

It is expected that the time required to obtain permission for exploration activity would be at least two months. For development activity six months would probably be required (Alamosa Planning Office, 1977). 


\subsubsection{Institutional Procedures}

The following is a detailed description of the institional requirements for geothermal exploration and development in Colorado.

Since the enactment of the Colorado Geothermal Resources Act in 1973 (C.R.S. 1973, 34-70-101) a few state agencies have adopted rules and regulations that specifically apply to the development of geothermal energy. Most of these regulations are derived from existing policies. Some agencies which could in the future exercise jurisdiction over the development of geothermal activities have not considered this resource potential. Very few counties have adopted regulations governing geothermal activity, primarily because of lack of interest or knowledge of the resource potential.

The Act assigns the authority of regulating all geothermal well activities in the state to the $0 i 1$ and Gas Conservation Commission (C.R.S. 1973, 34-70-104). There are, however, other state agencies and a few counties which issue permits that may be required for the exploration and the development of geothermal resources. The state regulatory agencies will be identified in this section. The activity regulated as well as the provisions and required procedures will be described. Included in this section will be flowcharts which depict the sequence of procedures that are to be followed by some of these agencies along with a brief summary of information on the institutional procedures.

The permit requirements for geothermal activities will vary, depending upon the ownership of the property and the extent of development. Some of the established regulations appear vague, primarily because they have not to date been applied to geothermal development. There are some inconsistencies in terminology among departments which will be clarified within this section.

"Geothermal resources" means geothermal heat and associated geothermal resources, including but not limited to:

(1) Indigenous steam, other gases, hot water, hot brine, and all other products of geothermal processes;

(2) Steam, other gases, hot water, hot brine, and all other products 
of geothermal processes resulting from water, brine, steam, air, gas, or other substances artificially introduced into subsurface formations; and

(3) Natural heat, steam energy, and other similar thermal energy in whatever form found in subsurface formations.

"Geothermal by-products" means any substances which remain after thermal energy has been removed from geothermal resources, including but not 1 imited to cooled waters, solution minerals, chemical compounds; extractable salts, rare earths, and other mineral substances.

\section{Oil and Gas Conservation Commission}

The Oil and Gas Conservation Commission is authorized to regulate all geothermal drilling activities within the state (C.R.S. 1973, 34-70-104). Prior to the drilling of any well, regardless of depth, a permit must be issued by the commission. This regulation applies to the drilling of an observation wel1, "a well used solely for informational purposes." The application for a permit to drill is filed with the Director, along with a filing and service fee of $\$ 75$. An accurate plat or map showing the location of the well must also be submitted. The developer will be required to post a plugging bond to insure that the well, upon abandonment, will be plugged in accordance with the commission's rules and regulations. Bond is set at $\$ 10,000$ per well or a $\$ 50,000$ blanket bond would cover all wells except that this bond would be waived where a bond has been filed in accordance with Federal or Indian lease requirements. An observation well permit may be processed in two weeks, although the law allows 60 days.

Further exploration by drilling requires the issuance of another permit. A written statement based upon data obtained from the observation well or from "similarly situated geothermal resource areas" must be submitted. The statement must include the following information:

1) Names and addresses of the owner, operator, and designated agents of both;

2) Location of the wells and proposed depth thereof; 
3) Description of the lease;

4) Amount and extent of surface development anticipated;

5) Measures taken to protect against land subsidence, contamination of surface and ground waters and the air, and excessive noise levels;

6) Proposed methods of geothermal by-product disposal and geothermal by-product recovery;

7) Mineral and chemical composition of any brine and associated gases of the geothermal resource;

8) Proposed casing program;

9) Any other information requested by the Commission.

Additionally, the developer must secure public liability insurance commensurate to the scope of the application.

The application and geological data is submitted to the State Engineer for review and comment. If the proposed exploration well is connected to a groundwater basin or a surface spring, the state Engineer must resolve the question of water rights before the permit can be approved. A permit must be obtained to excavate a retaining pit to store any substances produced from a we11.

Permission to plug a completed well or abandon a well must be obtained from the Director. Within 30 days after recompletion, plugging back, abandonment, formation fracturing, or other similar operations, the developer must submit a report to the Commission containing the following information:

1) A detailed account of the work done and the manner in which such work was performed;

2) The daily production of geothermal fluids and gases both 
prior to and after the operations;

3) The size and type of perforations;

4) The quantity and type of materials used in the operation;

5) Any other pertinent information or operations which affect the original status of the well.

Any retaining pits must be filled and the location cleared and restored to the satisfaction of the Director before the plugging report will be approved. Upon completion of the well, a Completion Report must be filed with the Director within 60 days, using a form that may be obtained from the Commission.

Should the geothermal well become a production well, producing a marketed resource, the developer would be required to submit monthly production reports. Additionally, a Certificate of clearance must be obtained to transport geothermal resources from a we11. Depending upon the operations of the production well, other reports may be required. However, it would not be possible to construct a completely accurate scenario of required procedures at this time due to the scarcity of geothermal activity within the state.

\section{State Engineer}

It is the responsibility of the State Engineer to preserve the water rights of others (C.R.S. 1973, 37-90-137). Prior to the issuance of a permit for a geothermal exploration or development we11, the State Engineer must determine that the construction of the well would not interfere with the water rights of others or the use of groundwater. After the well application and the geological data are reviewed by the $0 i 1$ and $G a s$ Conservation Commission, the application is sent to the State Engineer for comment: The proposed geothermal well may be connected-to a groundwater basin or a surface spring, in which case the question of water rights must be resolved before the permit can be approved. Tributary groundwater or surface streams are subject to appropriation (C.R.S. 1973 37-92- ). Applications for water rights to tributary water must be filed in the district water court. However, if the State Engineer is assured that the geothermal fluids will be reinjected after they are used, the well permit will be approved without a water right. A 
decree must be obtained from the court if the water will be diverted for other uses. If the proposed well is in a designated groundwater basin, the permit must be approved by the Groundwater Commission.

The use of non-tributary groundwater cannot deplete the water supply of others. In accordance with the law, a maximum of $1 / 100$ th of the water supply in the formation can be removed each year (C.R.S. 1973, 37-90-137(4)). There is a $\$ 25$ filing fee paid to the $O i 1$ and Gas Conservation Commission for a geothermal we11. The permit review process requires a maximum of 60 days from the date of application.

State Board of Land Commissioners

All geothermal leases on state-owned property are issued by the State Board of Land Commissioners (C.R.S. 1973, 36-1-112.(1) (q)). To obtain a state lease, an application is submitted to the Board. Notice of the requested lease is then sent to the respective county commissioners and planners, state, and federal agencies for comment and is publicly posted at least thirty days before the award of the lease is contemplated. The Board reserves the right to award leases on either a competitive or non-competitive basis. Should there be competition for a state lease, a public auction would be held. Leases have been issued on a non-competitive basis thus far because of the lack of interest in geothermal development. A minimum of two months is required to obtain a geothermal lease. The primary term (usually for 10 years) and annual rent are set by the Board. The filing and service fee is $\$ 75$. Currently there are no acreage limitations.

Before any drilling can be done, the Lessee must apply to the Board for an exploration permit. The Board's definition of an exploration well is one that is used to obtain geological and hydrological information only, or the type of well classified as an observation well by the $0 i 1$ and Gas Conservation Commission. To drill on any property within the state, a developer must obtain an observation well permit from the $0 i 1$ and $G a s$ Conservation Commission. The section entitled $0 i 1$ and Gas Conservation Commission details their requirements for issuing that type of permit.

To apply for an exploration permit, the Lessee must post an exploration bond for a minimum of $\$ 2,000$ per well to guarantee compliance with the Board's 
requirements for restoring the surface. Maps and other information concerning the planned exploration may also be requested by the Board. All drilling activity must be conducted in a maner that precludes contamination of fresh waters of the area or hazards to persons or livestock. Water disposal permits and air emission permits will be described in greater detail subsequently. The surface of each drill site must be restored to the Board's satisfaction before the developer will be released from liability under the exploration bond. A log of each well drilled on the leased land must be submitted to the Board. It must contain the following information:

1) The location, elevation, description of the formations passed through;

2) The depth at which each formation was reached;

3) The number of feet of each size casing set in each well and where it was set;

4) The total depth of each well.

Additional information must be made available upon the Board's request. Although there are established regulations with which to comply for further development of geothermal wells, there has not yet been a test case. According to the regulations, the Board must be kept informed of any proposed development. Prior to drilling a well for the production of geothermal resources, the developer would be required to post a development bond set by the Board. "Should the well become a geothermal production wel1, royalties must be paid to the Board. To date, no such wells have been drilled within the state. The minimum royalty rate would be $10 \%$ of the gross well head values, before transportation, paid monthly.

\section{Water Quality Control Commission}

The Water Quality Control Commission was established to adopt regulations for the state discharge permit system, designed to be in conformity with the Federal Water Pollution Control Act Amendments of 1972 and the Clean Water Act of 1977 (C.R.S. 1973, 25-8-501 through 508). Any geothermal by-product 
disposal, including thermal discharges, into state waters requires a discharge permit. There are two kinds of discharge permits; one for surface water, the other for subsurface water. The filing fee may vary from $\$ 10-\$ 250$, depending upon the extent of the proposed development. The developer must allow a minimum of 180 days for the application to be processed.

Public notice of the proposed discharge must be given and information about the location of the discharge is published in a Fact Sheet for circulation to various agencies and interested parties. Should any objections be filed, a public hearing must be held. The applicant will be required to pay for an expert's evaluation of the proposal if the discharge is to be injected into subsurface water. Surface water discharge applications but not subsurface injection applications must be reviewed by the Environmental Protection Agency. Permits are awarded for 2-5 years.

The pollution standards may be waived by the Commission if warranted by the economic feasibility of the development only for a proposed subsurface injection disposal but not for surface disposal. Determining the pollution standards for a development may vary under certain circumstances. For surface disposal, whenever the water quality of a stream is higher than a standard, the higher quality standard is to be maintained. The average stream flow is also taken into consideration.

\section{Air Pollution Control Commission}

Authorized to achieve and maintain the air quality in the state, the Air Pollution Control Commission has established standards with which a developer must comply (C.R.S. 1973, 25-7-108). The emission of hydrogen sulfide from geothermal wells can be a hazardous air pollutant and is a highly reactive substance that causes metal to corrode. Dust particles emitted from the drilling of wells are also considered to be potentially hazardous by the Division.

The developer is required to obtain either an emissions permit or a waiver prior to drilling an exploratory well. If it can be demonstrated that the hydrogen sulfide emission would be insignificant, the commission will award a waiver. In addition to a $\$ 40$ filing fee for the permit, the applicant must pay for an expert opinion on the estimated emission level. This information must 
be reviewed by the engineering staff before the permit can be issued by the division. The process requires approximately 20-90 days, depending upon the scope of the geothermal development. Emission measurements must be taken periodically.

\section{Public Utilities Commission}

Created to have "exclusive jurisdiction over all public utilities", the Public Utilities Commission regulates rates, services, and construction permits (excluding a municipality) (C.R.S. 1973, 40-3-102). The acquisition of a public utility by a municipality requires voter approval authorizing bonded indebtedness (C.R.S. 1973, 31-32-201).

To construct a power generation facility, electric or steam, the developer applies for a certificate of Public Convenience and Necessity. Preliminary approval will be based upon the commission's determination that there are sufficient financial resources for operations, existing utilities are unable to provide the power, and that it is beneficial for the state. Public hearings are then held. The developer must obtain the approval of the State Health Department, the State Land Use Commission, and the County Commissioners before final approval for construction. Although it is difficult to estimate the amount of time that would be required for approval in the future, the last permit application required $11 / 2$ years for approval.

The Public Utilities Commission's jurisdiction extends to the sale of public utility services to retail customers outside a municipality. If the service is sold on a wholesale basis to a town, the utility company would be under federal jurisdiction. Construction of a geothermal power generating facility which would provide services on a wholesale basis may require the approval of both the state and federal governments. It is a question that remains to be tested.

\section{State Land Use Commission}

The Land Use Commission was created to develop a statewide land use planning program (C.R.S. 1973, 24-65-104). To accomplish this goal, counties were required to adopt subdivision regulations and to file these with the commission. This requirement was met by the end of 1972 . Legislation was passed in 1974 which authorizes local governments to plan for and regulate the 
use of land within jurisdictional boundaries (C.R.S. 1973, 29-20-104). Very few counties have submitted updated subdivision regulations to the Land Use Commission. Although the law authorizes local governments to establish land use regulations, the counties are not required to file these with the state commision. The developer must therefore inquire about county zoning regulations in the respective county. Approval must be obtained from the county planning commission for the construction of a public building or utility (C.R.S. 1973, 30-28-111 through 209).

State Highway Department

A utility permit would be required for the use of or crossing of a state Highway with utility lines. This permit is obtained in the district engineering office. An agreement is signed by the utility company to restore the surface of the land excavated or to reimburse the State Highway Department for any damages and a private enterpreneur would be required to post bond to guarantee restoration of the surface. The contractor must comply with traffic control requirements. Issuance of a utility permit requires approximately one week.

\section{State Tax Incentives}

Although several bills were introduced during the 1979 legislative session to provide tax incentives for developing alternative energy systems, on $1 y$ two bills were enacted. S.B. 321 allows an adjustment to gross individual income on state income tax for the "design and construction costs which are in excess of conventional design and construction costs" of an energy system. The adjustment may be used whether the taxpayer is taking a standard deduction or is itemizing. S.B. 316 provides a property tax break for alternative energy systems that waives the inclusion of the energy system for property tax assessment for the years 1980 to 1989. However, once the deduction for the cost of the alternative energy system is applied for, the system cannot be depreciated along with other business assets.

Geothermal bills that were introduced during the 1979 session that did not pass include the following:

SB 324 - to require an annual appropriate from the severance tax trust 
fund to support the development and utilization of alternative energy sources in the state.

HB 1495 - to exempt alternative energy devices (including heating and cooling systems using geothermal) from sales tax.

HB 1540 - to exclude an alternative energy device from valuation of property for property tax assessment.

Following is a tabulation of leasing, permitting and regulatory requirements in Colorado. Following that are flow charts showing the procedures for the state Board of Land Commissioners, the State Engineer, the Water Quality Control Commission, and the $0 i 1$ and Gas Conservation Commission processing of applications. 


\begin{tabular}{|c|c|c|c|c|c|}
\hline Agencies & Permit & $\begin{array}{l}\text { Required } \\
\text { Prior to: }\end{array}$ & $\begin{array}{l}\text { Estimated } \\
\text { For Issuar }\end{array}$ & $\begin{array}{l}\text { Time } \\
\text { nce }\end{array}$ & Notes \\
\hline $\begin{array}{l}\text { County Plan- } \\
\text { ning Dept. }\end{array}$ & Zoning & $\begin{array}{l}\text { Depends on } \\
\text { county zoning } \\
\text { ordinances }\end{array}$ & variable & & $\begin{array}{l}\text { subdivision } \\
\text { regulates exist } \\
\text { in all counties }\end{array}$ \\
\hline $\begin{array}{c}\text { State } \\
\text { Agencies }\end{array}$ & & & & & \\
\hline $\begin{array}{l}\text { Board of Land } \\
\text { Commissioners }\end{array}$ & $\begin{array}{l}\text { Lease state } \\
\text { lands explo- } \\
\text { ration permit }\end{array}$ & $\begin{array}{l}\text { use of lands } \\
\text { exploration } \\
\text { of land }\end{array}$ & $\begin{array}{l}2 \text { mo. } \\
2-8 \text { wks. }\end{array}$ & & $\begin{array}{l}\text { May be competi- } \\
\text { tive bidding }\end{array}$ \\
\hline $\begin{array}{l}\text { Oil \& Gas } \\
\text { Conservation } \\
\text { Commission }\end{array}$ & $\begin{array}{l}\text { observation } \\
\text { well permit/ } \\
\text { exploration } \\
\text { well permit/ } \\
\text { development } \\
\text { well permit }\end{array}$ & $\begin{array}{l}\text { drilling well; } \\
\text { deepening ob- } \\
\text { servation well; } \\
\text { drilling devel- } \\
\text { opment well }\end{array}$ & $\begin{array}{l}2-8 \text { wks; } \\
\text { approx. } 8 \\
\text { approx. } 8\end{array}$ & $\begin{array}{l}\text { wks: } \\
\text { wks. }\end{array}$ & \\
\hline $\begin{array}{l}\text { Water Quality } \\
\text { Control Comm. }\end{array}$ & $\begin{array}{l}\text { surface dis- } \\
\text { charge per- } \\
\text { mit; injec- } \\
\text { tion discharge } \\
\text { permit }\end{array}$ & $\begin{array}{l}\text { disposal of } \\
\text { fluid dis- } \\
\text { charge }\end{array}$ & $\begin{array}{l}6 \text { mos. } \\
6 \text { mos. }\end{array}$ & & $\begin{array}{l}\text { In conjuction } \\
\text { with EPA review }\end{array}$ \\
\hline $\begin{array}{l}\text { Air Pollution } \\
\text { Control Comm. }\end{array}$ & $\begin{array}{l}\text { Emission } \\
\text { permit }\end{array}$ & $\begin{array}{l}\text { discharge air } \\
\text { pollutants }\end{array}$ & $20-90$ days & & $\begin{array}{l}\text { developer must } \\
\text { obtain a waiver }\end{array}$ \\
\hline $\begin{array}{l}\text { District } \\
\text { Water Court } \\
\text {, }\end{array}$ & $\begin{array}{l}\text { Appropria- } \\
\text { tion of water }\end{array}$ & use of water & $?$ & & $\begin{array}{l}\text { most water } \\
\text { rights over } \\
\text { appropriated in } \\
\text { state }\end{array}$ \\
\hline $\begin{array}{l}\text { Groundwater } \\
\text { Commission }\end{array}$ & $\begin{array}{l}\text { Appropria- } \\
\text { tion in } \\
\text { designated } \\
\text { ground water } \\
\text { basin. }\end{array}$ & use of water & $?$ & & \\
\hline Highway Dept. & $\begin{array}{l}\text { Utility } \\
\text { permit }\end{array}$ & $\begin{array}{l}\text { use of state } \\
\text { highway for } \\
\text { utility lines }\end{array}$ & 1 week & & $\begin{array}{l}\text { would be neces- } \\
\text { sary for use of } \\
\text { or crossing of } \\
\text { state highway } \\
\text { w/utility lines }\end{array}$ \\
\hline $\begin{array}{l}\text { Pub1ic } \\
\text { Utility } \\
\text { Comm. }\end{array}$ & $\begin{array}{l}\text { certificate } \\
\text { of conveyance } \\
\text { and necessity }\end{array}$ & $\begin{array}{l}\text { construction } \\
\text { of a power } \\
\text { plant - sale } \\
\text { of electricity }\end{array}$ & $?$ & & $\begin{array}{l}\text { approval of St. } \\
\text { Health Dept., } \\
\text { St: Land Use } \\
\text { Conm., and } \\
\text { County Commis- } \\
\text { sioners also } \\
\text { required }\end{array}$ \\
\hline
\end{tabular}


FIGURE 3

PROCEDURES FOR OBTAINING GEOTHERMAL PERMITS

Oil \& Gas Conservation Commission (OGCC)

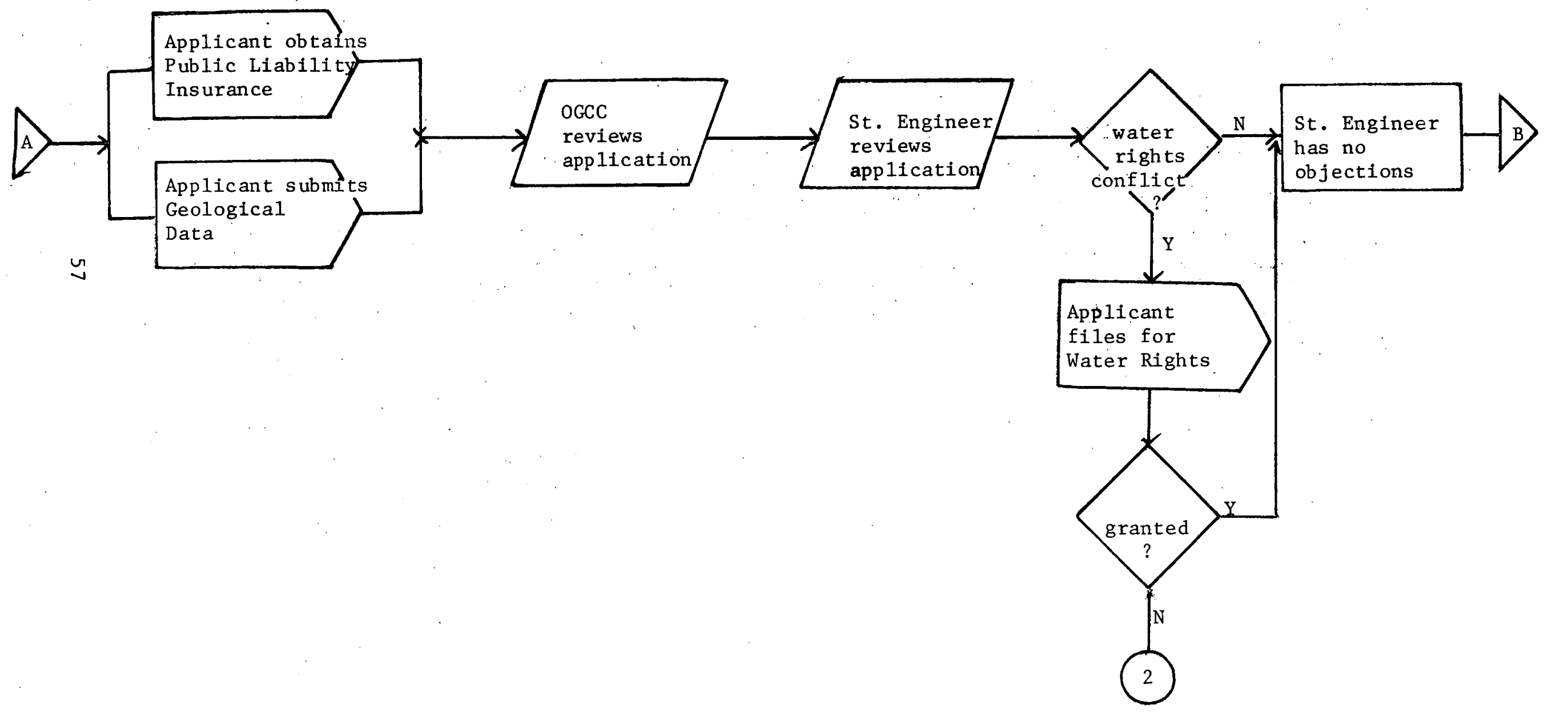




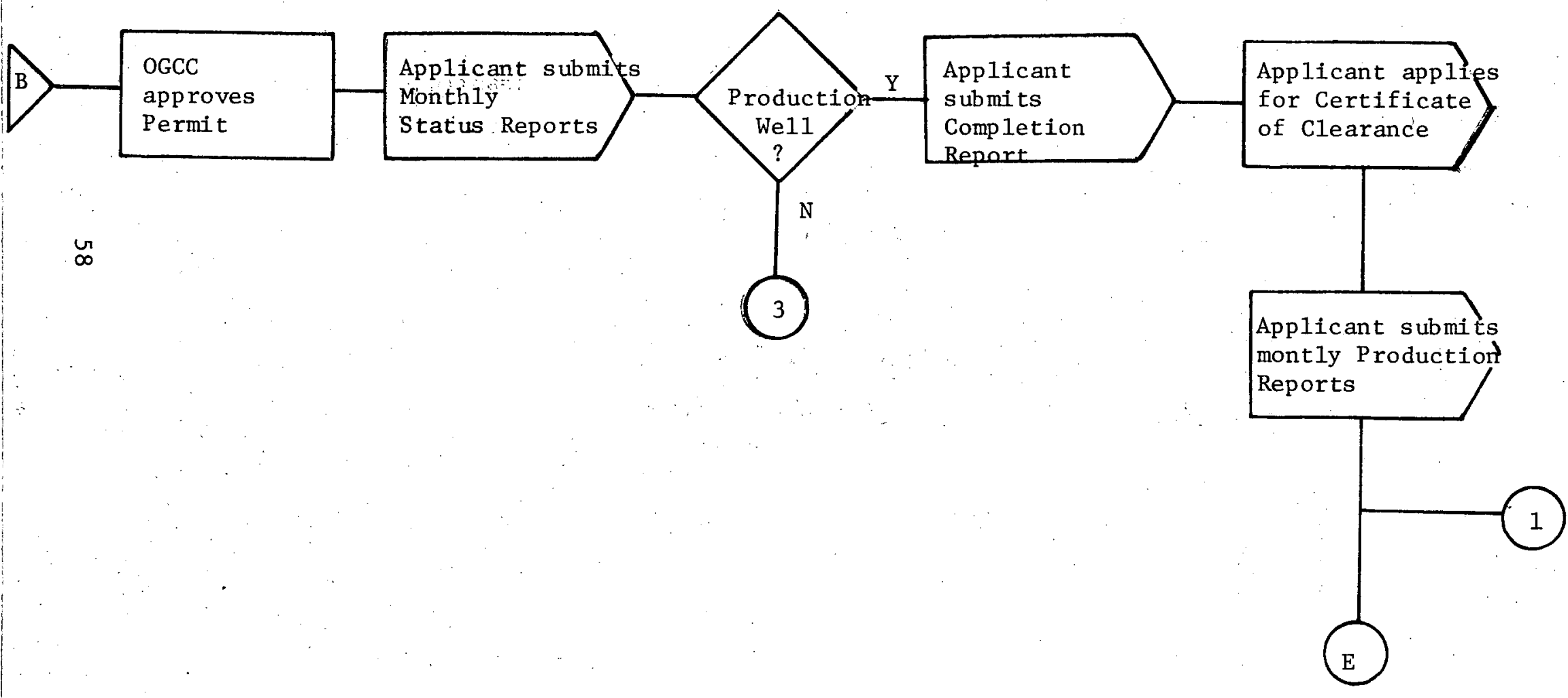


State Engineer Department

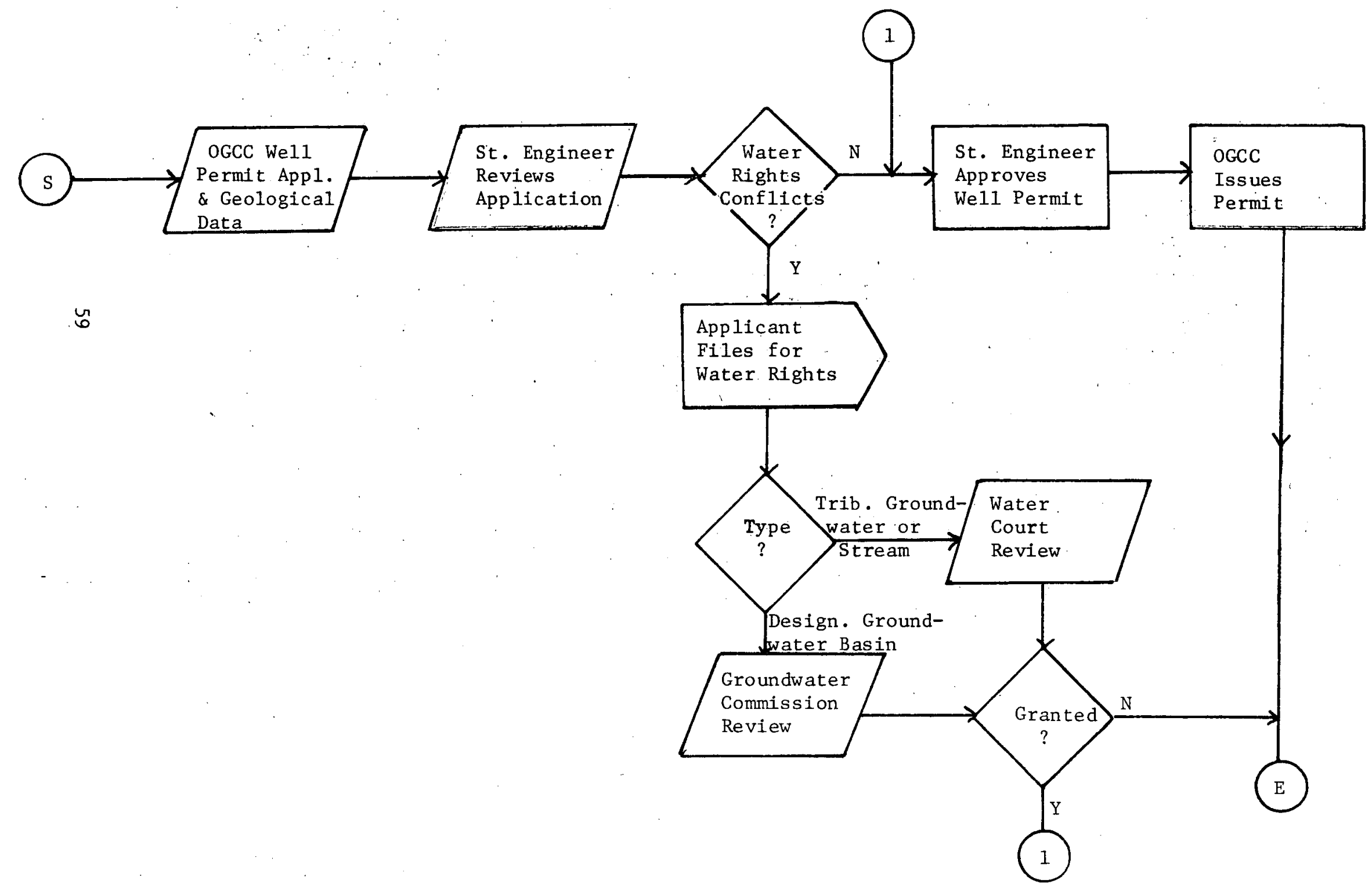




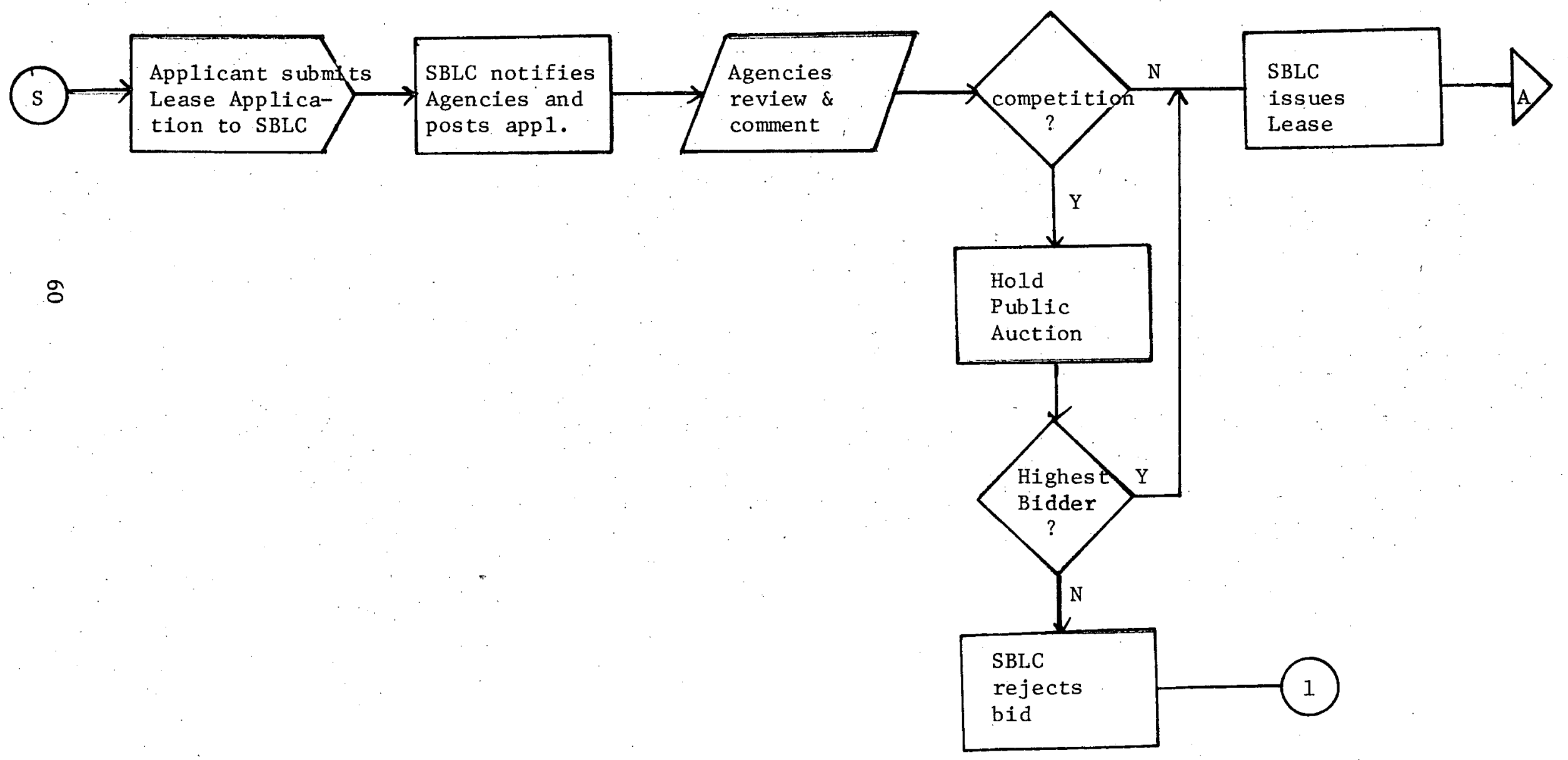




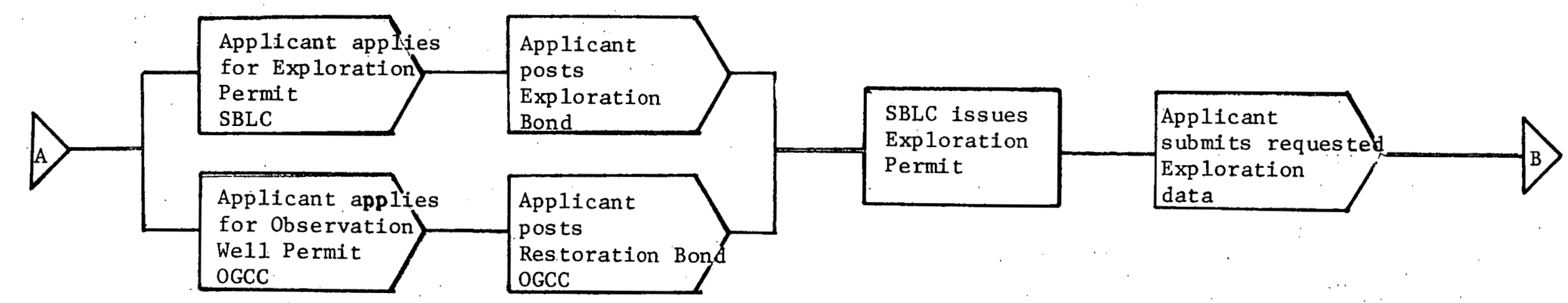




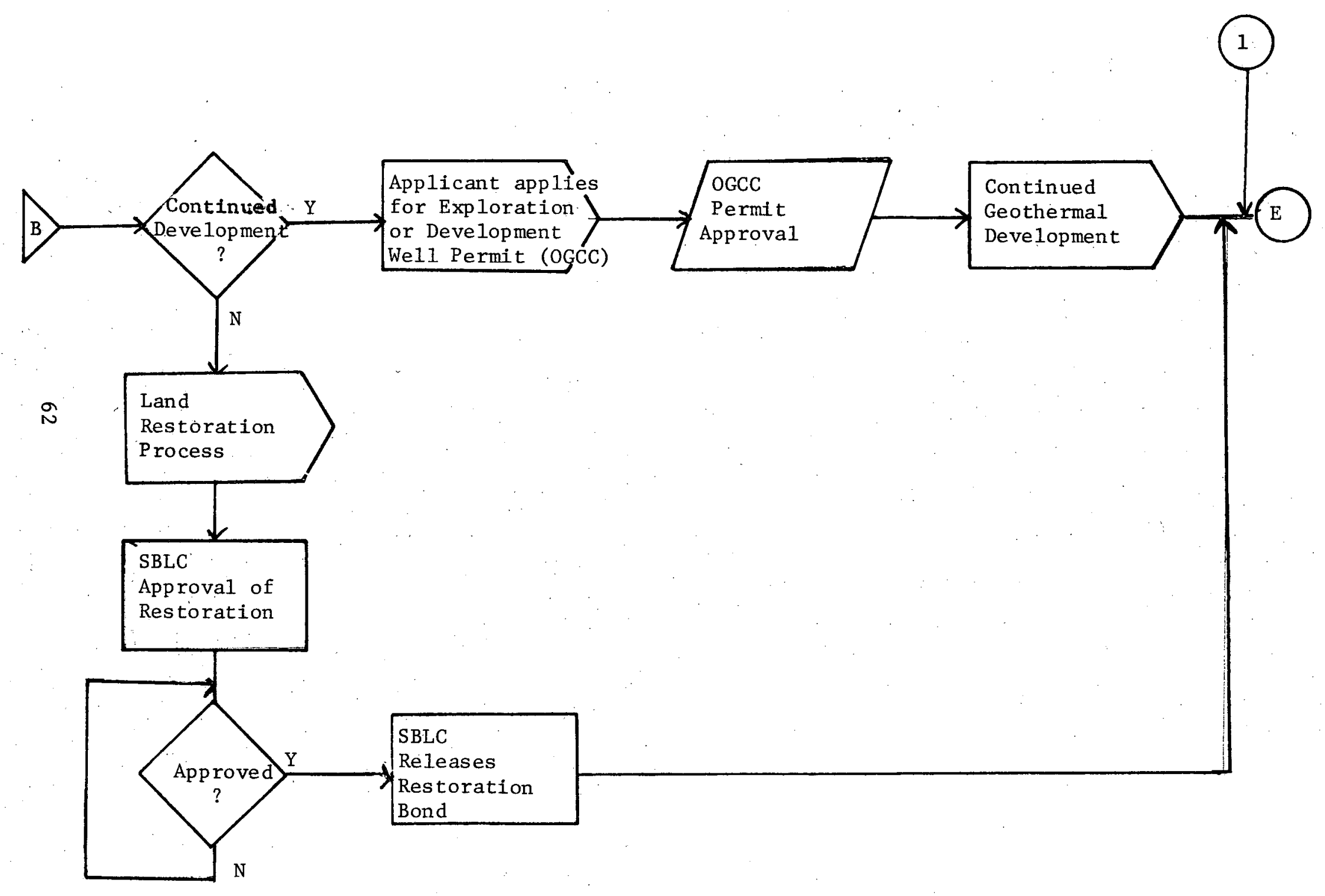


FIGURE 6

PROCEDURES FOR OBTAINING DISCHARGE PÉRMITS

Water Quality Control Commission (WQCC)

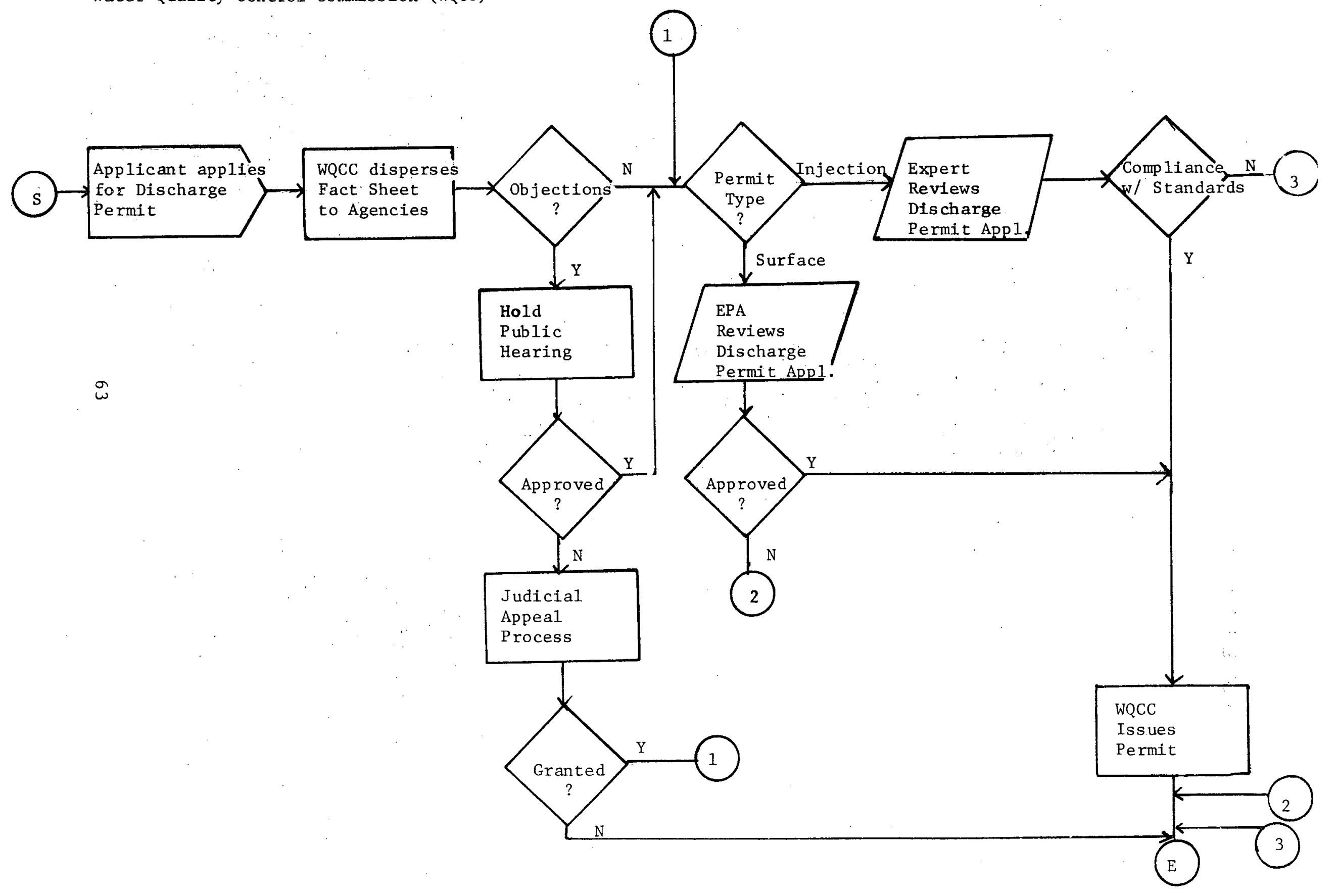




\subsection{Public Outreach Program}

\subsubsection{Outreach Mechanisms}

\subsubsection{Existing}

The outreach program is designed to provide information to help accelerate geothermal energy development. A number of methods for promoting the use of geothermal energy in Colorado were tried on an experimental basis. The best approach or combination of approaches may then be adopted for a continuing program. The methods are as follows:

3.7.1.1.1 - Media Publicity - news media, including TV, radio, newspapers, and business weeklies provide coverage. Numerous reporters have conducted interviews with Harris Sherman, the Director of the Department of Natural Resources; John Rold, Director of the Colorado Geological Survey; R. H. Pear 1 , Team Leader of the geothermal project, and Barbara Coe, Data Leader for that project. These are most often responses to media requests, since timely publicity activities of this office are limited by the need to direct new releases through several supervisors to the publicity officer. Furthermore, the item must capture the attention of the news media, not always possible, given the magnitude and exigency of competing current energy news. The large and varied audiences of news media publicity are an advantage to this approach. Some feed-back occurs through phone calls and visits following news media presentations, but for the most part, the results are not measurable.

\subsection{Meetings and Telephone Interviews}

Meetings with groups or individuals as well as telephone conversations are held to describe the energy potential development activities. or related topics. Information interchange is emphasized. Providing and obtaining information during the same effort is an efficient use of time. Furthermore, it is a more subtle approach than the promotional ones that sometimes are met with suspicion and antipathy. Meetings or interviews include those with industry, government agencies and communities, and individual users, as listed below.

\subsection{Industry and Agribusiness Contacts - Contacts were made with}


industry representatives, including existing and potential geothermal users, suppliers, and developers and technical consultants. They were invited to attend meetings, as well as being contacted for an exchange of information by telephone and in person. Some became known through conversations with others, while some are located simply by visiting geothermal sites and the areas surrounding them.

Information obtained included general energy needs, locational criteria, industrial processes, and financing methods. Information provided included sources of financing, techniques for development, availability of markets, characteristics of the resources, and sources of additional assistance. The summary listing in 3.7 .2 details these contacts.

3.7.1.1.2.2 Interagency Coordination - Contacts were initiated with other agencies to describe the geothermal project and the geothermal potential. Coordination and cooperation. procedures, including a communication network, were established when appropriate. At the same time, institutional investigations were conducted to learn about the other agencies' responsibilities and procedures directly or indirectly related to geothermal energy development.

3.7.1.1.2.3Communities and User Contacts - These contacts included communities, non-profit organizations, and potential individual users located near geothermal resources. They were given information about resources, potential uses, other geothermal developments, financial sources, development techniques, and information sources. They provided information about growth, economic conditions, planned or proposed development, goals and attitudes of the area's residents; institutional and other requirements for geothermal development in this area.

\title{
$3: 7.1 .13$ Lectures
}

Formal talks concerning the geothermal resource potential were presented on several occasions, including those at:

\author{
Western State College \\ Jefferson County Energy Fair \\ Colorado State University
}




\section{Senator Haskell's Energy Fair}

Such talks provide contacts with a specialized group, and at the same time include an opportunity to obtain feedback.

\section{7 .114 Workshops}

One workshop was held in. Denver to bring potential users together with engineers to discuss the feasibility of various proposed projects as well as possible solutions to technical problems. Such workshops are valuable, but require the preliminary outreach work to locate and generate users.

\subsubsection{Displays, Brochures, and Papers}

Brochures were prepared and widely distributed to county officials, energy fairs, other energy offices, and individuals. A display showing resource areas, equipment, and uses was shown at energy fairs. Papers discussing the potential for geothermal development were submitted to the American Planning Association, the Solar Energy Research Institute Community-Scale Renewable Energy Systems Program, and the Geothermal Resources Council. Because of the comparative lack of feedback, the results obtained from these efforts are not measurable. All of these activities reach a large audience. Papers, however, are very time-consuming to prepare and are not always selected for presentation or publishing.

\subsubsection{Summary Listing of Contacts and Results}

\section{Contact}

\section{Industry}

Eaton Develop. Corp., Archuleta County
Prospective Application

\section{Comments}


Denver Business Week

Riverbend Estates,

Development

Petroleum Information

general

Willard Owens, Developer heat homes

Hazen Resources

Grand Junction Sentinel general

Indian Springs Resort heat resort

(in town of Idaho

springs)

Landowner, Chaffee

County

Acme Plumbing \&

Heating

Coury \& Assoc.

Lawrence Ross,

Consulting Engineer

Energy Materials, Inc. heat homes

general

general

article in weekly

said would drill here, but have not yet

report on geothermal

activities

doing feasibility study

for heat-pump application

Discussed geothermal

resource potential in

Colorado. general

space heat

Got tech assistance from

$E G \& G$. May not be

economical.

interested in value of

geothermal resource

Do hot water, and heat pump systems. Could do geothermal.

Coordinate efforts to encourage geothermal development.

may submit PRDA proposal

heat Glenwood

Springs bank

general have plastic pipe useful in geothermal systems 
Clark Millison,

Consulting Geologist

Whetstone, Inc.,

builders

LOPA Hydrotheraphy

Rico Argentine Mine

Solar Pathways

\section{Agribusiness}

Gary Weisbart

Doug Sutton

Vicki Hays

Executive Director, Colorado Flower Growers Association. district heat

heat homes

heat additional

structures

mineral extraction, heat company homes

district heating

swine houses, suiefers, methane plan, fish culture

greenhouse

greenhouse

heat greenhouses

heat greenhouse

Gindy did review of Glenwood

Springs geology

interested, need

information about

resources, grants

need engineering help, grant

Interested in developing. Have wells already. Mine and mill may be sold.

Cannot invest money until option is either exercised or dropped.

might submit PRDA constructing pens, having engineering done for methane plant

need front-end funds

need front-end funds

said studied previously, not feasible
Said energy problem, but energy conservation measures help. Said imports are a bigger problem. 
Rainbow Trout Farm

Roger Sherman

Individuals

Linda Newhall

Ouray Citizen's

Committee

Glenwood Springs

Citizen Committee fish culture

greenhouse

heat homes

district heat, industrial or

agricultura1 processes
Said market good for

catfish. Trout need low temperature water. Their trout market is primarily for stocking.

needs grant, technical assistance said well doesn't have enough flow, clogs pipes

need clarification of legal aspects, resource assessment, we 11 , feasibility study, funds, but can't match federal grants

district heat, industrial or agricultura 1 processes
Need resource assessment, we11, clear definition of geothermal rights in

Glenwood Springs, frontend financing for system. Said minimal opportunity for industry.

Federal Government

Regional office; U.S.

general

Requested reports

Dept. of Commerce

as published. As governor, director had promoted geothermal energy. 
Regional Office, DOE

DOE Solar Energy

Research Institute

U.S. Forest Service

Bureau of Land Mgmt.

\section{State Government}

State Legislature

Div, of Commerce \& Dev.

Four Corners Regional

Commission

Economic Development

Planning

Division of Housing

0il \& Gas Conservation

Commission

State Land Board

State Engineers office general

Energy Conservation

office

Dept. of Institutions

process

$$
\text { general }
$$

industrial processes

general

genera 1

genera 1

process

process

heat 1ow-cost housing

general

general

alternative energy

heat new prison, use

for prison industries
Discussed schools \&

hospitals grant and loan

guarantee. Approved

technology and smal1

grants programs.

Discussed energy

demand studies \& needs 
Director's office,

general

Dept. of Natural

Resources

Governor's office

Dept. of Highways

Colo. Energy Research

Institute general

snow \& ice melt

requested feasibility study

gasohol, heat State buildings coordinated study 
Mayors and other officials of the following municipalities:

$\begin{array}{lll}\text { Craig } & \text { Salida } & \text { Pitkin } \\ \text { Steamboat Springs } & \text { Poncha Springs } & \text { Ouray } \\ \text { Idaho Springs } & \text { Canon City } & \text { Telluride } \\ \text { Glenwood Springs } & \text { Florence } & \text { Durango } \\ \text { New Castle } & \text { Lincoln Park } & \text { Paradox } \\ \text { Carbondale } & \text { Buelah } & \text { Norwood } \\ \text { Hotchkiss } & \text { Del Norte } & \text { Naturita } \\ \text { Paonia } & \text { Hooper } & \text { Nucla } \\ \text { Crested Butte } & \text { Alamosa } & \\ \text { Buena Vista } & \text { Pagosa Springs } & \end{array}$

Other Non-Profit Organizations

Councils of Governments or Regional Planning Commissions of the following regions :

$$
\begin{aligned}
& \text { 3. Denver } \\
& \text { 8, San Luis Valley } \\
& \text { 9, San Juan Basin } \\
& \text { 10, Black Canyon } \\
& \text { 11, Northwest }
\end{aligned}
$$

Whiteman School space heat - Need resource information, grant.

Timberline Academy space heat - Need grant for system development.

Local Government

Board of County Commissioners of the following counties:

$\begin{array}{ll}\text { Moffat } & \text { Chaffee } \\ \text { Routt } & \text { Fremont } \\ \text { Jackson } & \text { Pueblo } \\ \text { Grand } & \text { Saguache } \\ \text { Boulder } & \text { Alamosa } \\ \text { Clear Creek } & \text { Conejos } \\ \text { Eagle } & \text { Archuleta }\end{array}$




$\begin{array}{ll}\text { Garfield } & \text { Mineral } \\ \text { Pitkin } & \text { Ouray } \\ \text { Delta } & \text { San Miguel } \\ \text { Gunnison } & \text {. Dolores } \\ \text { Park } & \text { La Plata }\end{array}$

County Planning offices of the following counties:

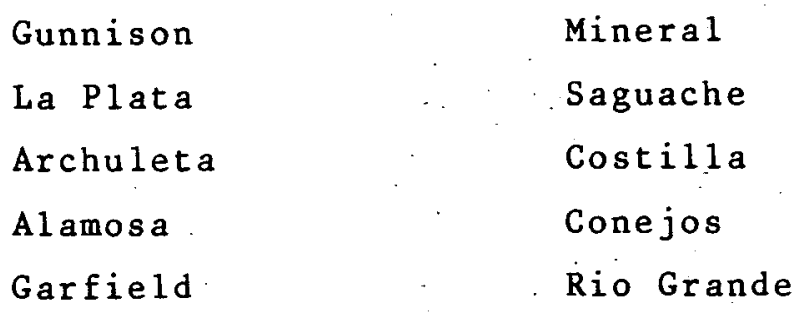

\subsubsection{Recommended Mechanisms}

Media presentations reach the largest number of people, although it is unknown how much of this information is absorbed. Increased emphasis in the form of more frequent releases is planned. A professionally-prepared attention-getting media display or presentation would probably be helpful, but is not within the means of this project.

Meetings are especially helpful in that questions can be answered and feedback obtained regarding the acceptance of geothermal energy, the level of development interest, and constraints to development. Several people can be contacted at once. Telephone interviews help, but can only reach one person at a time and lack the depth of conversation. Increased emphasis upon meetings is planned. The audiences emphasized will be slightly different, however, since some have been sufficiently covered. County governments will be deemphasized in most cases. Industry and Chamber of Commerce contacts will be increased. For the most part, interagency coordination will continue as it is, since the communication network has been set up. Some additional federal agencies including regional offices of Housing and Urban Development, Department of Transportation, Department of Agriculture, Health, Education and Welfare, Economic Development Administration, and the Federal Regional Council will be provided with basic information and asked for information about possible mutual 
efforts.

Municipal and user contacts will continue at about the current level. However, among those municipalities and potential users that have passed the initial awareness of geothermal energy stage, the need for feasibility studies, detailed resource assessment, and development funds are apparent. While these kinds of assistance are extremely limited, the Colorado Team attempts to guide communities and potential users to whatever help is available.

Lectures are especially beneficial, since large audiences can be reached and at the same time, feedback can be obtained. At least one additional paper is planned for the Governor's Environmental Geology Conferences. Little time will be spent on others, because their acceptance is often not assured. The display and brochures will continue to be distributed.

Additionally, an industry survey would be in order to obtain information about industry needs and interest. A central or coordinated effort by all states involved in this project might be more valuable; since state boundaries are not a critical factor in industry relocation decisions.

\subsubsection{Overa11 Prospectives for Future Geothermal Activity}

The opportunities for geothermal development in some areas in Colorado seem strong, if the opportunities are grasped. Of the 60 areas so far identified in Colorado, only 3 or 4 seem to have potential for power generation. Many more areas offer potential direct thermal resources and markets. In several resource areas, new energy and other mineral developments will occur. In most of these, the population influx will represent a significant percentage increase over the current population. Geothermal energy could heat new housing and commercial structures, thus easing the burden on existing utilities and perhaps lowering heating costs for users.

Areas with high recreation and tourism activity are experiencing growth, as wel1. Facilities such as motels and restaurants use large quantities of hot water and heating fuel that could readily be provided by geothermal energy.

Still a third type of development opportunity might be possible. Some areas of high unemployment and low incomes could offer geothermal energy as an incentive 
to encourage new industry to locate in their areas. Such areas as the San Luis Valley have an agricultural base that would lend itself to agribusiness uses of geothermal energy. The timber industry, although declining, has been an important industry that might find geothermal kiln-drying to be practicable. The mining industry, another historically active and currently growing strong industry in the State, might find geothermal energy useful for mineral

extraction. Other industry, particularly manufacturing, near Colorado's identified resource areas, is limited. Because of mountain terrain, lack of transportation and other locational criteria, extensive new industrial development is not likely in most resource areas of Colorado.

Colorado's most pressing energy need is energy for space and water heating. With communities growing because of both. recreation and energy development, these needs will grow. Natural gas has been limited in the State until the recent Federal policy change. Propane and electricity have been the primary alternative fuels, at high cost.

The opportunity and the need exists, but developers are lacking. Growing communities are burdened with critical needs for housing, water systems, sewer systems, streets and public services, as well as utility systems, and local government resources are extremely limited. Furthermore, federal grants are usually available for community improvement projects and are thus expected. Too, private utilities have supplied energy for many communities. Given the abundance of needs, and the scarcity of resources, along with the lack of experience with utility systems, communities are likely to postpone the development of geothermal systems. Nor do energy producing companies express the interest in development of geothermal energy for direct use as in power generation. Given these development constraints, the potential for geothermal power generation in Colorado may be realized long before the direct thermal potential is even tapped. 


\subsection{Summary of Major Findings and Recommendations}

The objectives of this study are to reveal the potential for geothermal energy development in Colorado, current constraints to that development and ways those constraints might be removed. Toward this end, data from numerous published documents were analyzed. Sites were visited and conversations were held with prospective users, developers, public officials, residents, and other participants. Possible development was then postulated from this information. These activites resulted in the findings and recommendations described below.

\subsubsection{Electrical Power Potential}

Colorado seems to have geothermal potential for electrical power generation at 4 or 5 sites of the 56 sites inventoried by the State coe, 1978). Some analyses have attributed no such potential to the State. However, conversations with representatives of energy companies who hold leases at Poncha Springs, Waunita, Mineral, Chalk Creek, and Cebolla in particular indicate possible high temperatures have been inferred from preliminary exploration data. At the site with the highest measured temperatures in the State, Chalk Creek, Hortense Hot Springs as well as a 180 foot deep well, have measured surface temperatures of $82^{\circ} \mathrm{C}$ (Barrett and Pear 1,1978 ).

The inability to obtain leases on National Forest, for which applications were submitted in 1974, has stymied exploration in at least two areas, Chalk Creek and Poncha Springs. The U.S. Forest Service is just now preparing an environmental assessment report for the Chalk creek leasing, with October, 1979, the earliest that completion of that report is expected (William Dolan, pers. comm.). Additionally, the B.L.M. has denied Amax's request to issue leases on BLM land prior to the U.S.F.S. decision.

In one area, Cebolla, necessary leases on private land have been thus far unobtainable (Butler, pers. comm.) It is believed that if these leases were to be issued, given the tax incentives of 1978 , exploratory we 11 s would be drilled and the potential of these areas further defined. Given conflicting mandates among various federal agencies, however, the Federal lease acquisition barrier may remain. Nothing short of condemnation can force issuance of leases on private land. However, educational programs could help encourage willingness 
to lease.

4.0.2 Direct Thermal Potential

Because of the low and moderate temperatures, the greatest geothermal potential in Colorado is for direct thermal uses. Markets for the energy seem to be readily available near the resource sites. At least 30 communities are within a reasonable distance for using the energy. Twenty-three of these communities are within 10 miles of a resource area; sixteen are virtually on-site (Coe, 1978 ).

Of this direct thermal potential, most is for space and water heating. In 17 of 24 communities visited, space heating, not including greenhouses, is the predominant demand for geothermal energy. The reasons are clear, when one examines the economy of colorado.

As Table 9 shows, the largest employment sector in the State is wholesale and retail trade, with government next and services third. All categories except agriculture show an increase. The first and latter categories reflect the strong emphasis of tourism in the State. Because these categories are larger does not mean that the other categories are insignificant, however. Determining the industries relevant to direct use of geothermal energy can only be accomplished by examining the industrial patterns and potential near geothermal resource areas. Those areas that have been identified thus far from surface expressions are all in the western two-thirds of the state, primarily along the mountain range.

Agriculture is an important industry in Colorado, but half the production comes from 5 counties on the eastern plains. All of the prime land identified by the State Department of Agriculture lies in the eastern plains. Agricultural areas are, however, scattered around the West Slope and concentrated in the San Luis Valley, an area that seems to have especially high potential for agricultural processing (Colorado Division of Planning, 1.978). Other areas may have such potential as well, where production levels are sufficiently high to warrant plant construction. Additionally, if sufficient timber continues to be produced from National Forests, manufacture of wood products could offer an opportunity for development of geothermal energy. 
STATE OF COLORADO ANNUAL AVERAGE EMPLOYMENT DATA*

\begin{tabular}{lrrrr} 
& \multicolumn{3}{c}{ Resident Labor Force } \\
& 1970 & 1975 & 1977 (estimate) \\
Total Employment & 873.4 & $1,088.8$ & $1,158.8$ \\
Agriculture & 55.5 & 50.2 & 50.0 \\
Mining & 8.8 & 18.3 & 22.1 \\
Contract Construction & 40.1 & 56.8 & 55.8 \\
Manufacturing & 117.7 & 134.9 & 145.7 \\
Transportation \& Public Utilities & 51.6 & 59.8 & 63.0 \\
Wholesale and Retail Trade & 173.1 & 236.2 & 255.4 \\
Finance, Insurance and Real Estate & 39.3 & 56.1 & 61.1 \\
Service & 135.1 & 183.3 & 204.0 \\
Government & 165.6 & 210.2 & 218.2 \\
(totals will not add due to rounding and omission of "other" category) \\
*All numbers are in thousands.
\end{tabular}

Source: Research and Analysis Section, Colorado Division of Employment and Colorado Business/Economic Outlook Committee. 
Manufacturing is another significant industry in Colorado but almost two-thirds of the 245 new industries that were announced between 1970 and 1976 were planned for the Front Range.? About 92 percent located near a community of 20,000 population or more (Colorado Division of Planning, 1976). Most communities near geothermal areas in Colorado are much smaller. However, where small communities such as Ouray are attempting to encourage the initiation of light industrial units, geothermal energy has a potential industrial market, as we11. as helping to encourage such activity.

That Colorado is rapidly becoming an energy resource center is apparent by the huge energy office buildings proliferating in Denver. Extensive deposits of coal, uranium, and oil shale, as well as some oil and gas, are found in the State. Furthermore, as evidenced by the location of the Solar Energy Research Institute in Colorado, Colorado is a prime area for use of solar energy. Population influxes. generated by development of these resources require expansion of utility systems including heating. Much of the energy fuels and metallic minerals are found in the western part of the state, many of them near geothermal areas. Use of the geothermal energy for heating in such areas as Gunnison County can help alleviate negative socio-economic impacts of energy and mineral development.

Probably the best known economic resources in colorado, however, are its environmental attractions that attract both permanent settlers and visitors. In particular, forested mountains with 54 peaks over 14,000 feet high are legendary. They provide a multitude of opportunities for outdoor recreation including hiking, fishing, hunting, skiing, boating, and sight-seeing. Control by the Federal government of over 24 million acres of the 66 million acres in the State creates opportunities and restrictions. Since nearly all of the Federally-held land is in the western two-thirds of the state, the kind and extent of development that occurs is largely dependent upon the Federal government.

Tourism and recreation are increasingly important to Colorado; with the State attracting over 12 miliion visitors per year (Division of Planning, 1978 ). Many communities, especially on the Western slope, rely upon tourist dollars to sustain them (Division of Planning, 1978). Continued increases in tourism and recreation facilities seem likely, unless curtailed by shortages or fears of shortages of gasoline. Lodging and food establishments require huge amounts of 
heat and hot water. Communities such as Durango, Pagosa Springs, Ouray, Steamboat Springs, and Glenwood springs that rely heavily on tourism and recreation seem to afford good opportunities for commercial use of geothermal energy as well as accompanying residential use.

Historical and existing economic patterns are not the only determinants of probable economic development. However, those conditions that led to current patterns are not likely to change dramatically. The Federal land ownership, rugged terrain, short growing seasons, and remoteness, as well as the desire among the residents and others to retain the existing environment precludes the development of major industrial complexes in most Western slope communities. Some scattered, small industrial and agricultural facilities will most likely afford opportunities for some industrial use of geothermal energy.

Even with these limitations, geothermal energy could be widely used in colorado where tourism and energy development strain energy supplies or energy user's pocketbooks. Two locations, the San Luis Valley and Pagosa Springs were subjected to detailed analysis to learn what their geothermal energy development potential might be. Major findings are as follows:

Unlike most areas of the State, in the San Luis Valley, the major opportunities for geothermal development focus upon agribusiness. Current uses of the energy such as livestock pen warming and aquaculture could be expanded and new uses such as greenhousing and agricultural processing could be introduced. The primary challenge is to locate prospective entrepreneurs who will establish these kinds of facilities and can obtain the necessary financing to do so.

Supply of residential and commercial space and water heating fuel was postulated, too, in the analysis. Studies by NMEI indicated that although the City of Alamosa could obtain the geothermal energy at a favorable price, not many such systems in the Valley seem to be economically feasible now. Development of such systems is limited, too, by the lack of interest of homeowners in conversion and by the lack of funds. Were funds available and savings, in energy costs demonstrated, these communities could be much more interested in the energy potential.

In Pagosa Springs, the primary current energy demand is decidedly for residential and commercial space and water heating. If the lumber business 
revives, that industrial activity may afford opportunities for geothermal energy use. If the planned district heating system there performs satisfactorily and economically, the use of the energy by suburban areas seem likely.

Although the geothermal energy has been used for years in Pagosa Springs, expanding its use required outside assistance. Although the interest in development was high, the means for development were lacking. The State's integrated geothermal program funded by DOE was instrumental in stimulating development. The community meetings held as part of the outreach program helped to consolidate the local interest. The resource assessment program helped to further define the possibilities of the energy resource. Then, through the outreach program, consultants were encouraged to help the community prepare a grant proposal. With the resultant funding, Pagosa Springs will be able to develop their geothermal energy. It is highly improbable that the City would be as far along in the development stage as they are were it not for those DOE programs.

Interest in use of geothermal energy is high but in only one community, Pagosa Springs, is the energy scheduled for development. These investigations reveal several reasons why geothermal energy has not been developed. Foremost among them is that the energy was not needed until recently. Natural gas and propane were available at reasonable cost and were clean, convenient and easy to use. Rising fossil fuel prices stimulate energy consumers to look to alternatives, particularly alternatives whose prices will remain reasonably stable.

While many of those alternatives have been widely discussed, geothermal energy has lacked extensive publicity. Among the general population as well as high-level decision makers, the words "geothermal energy" have seldom been heard. In spite of the lack of publicity, however, an unexpected level of interest in geothermal development is apparent in those areas where geothermal surface expressions are found.

Technological problems, especially problems resulting from corrosion and scaling, have discouraged development in the past. Information that new methods are available for dealing with those problems must be disseminated, often to skeptical audiences. Site-specific conditions often require site-specific system designs and treatments, adding to the difficulty and cost of 
development.

These problems are compounded by the lack of information about the depth, extent, temperatures and flow rates of the resource areas. Lacking such information, many are skeptical about the resource value. They may even consider only the visible hot water as the total energy potential. others hesitate because of uncertainty concerning the availability, magnitude or exact location of a resource.

The fifth but most influential constraint to geothermal development for direct use is the large capital investment and accompanying expertise required to explore, dri11, and construct a system for and market geothermal energy.

Established companies who have the expertise and capital to develop these resources have shown 1 ittle interest in them. The primary reason for this lack of interest is the apparent lack of opportunity to obtain a sufficient rate of return on investment from their development to compete with other available investments. Widespread energy company interest in direct use of geothermal energy is deterred, too, by the need to market and distribute the energy. An investment of this magnitude requires a sizeable market such as large commercial or industrial facilities or a community or subdivision, with price dependent upon using the resource efficiently. However, the market is limited geographically, thereby complicating marketing possiblities. Furthermore, the operation of a distribution system is not a normal producer activity. Nor is the small-scale of most propsective direct use geothermal applications in Colorado consistent with the usual operations of energy companies.

This lack of appeal of direct geothermal development to established energy companies leaves the development to either users or smal1-scale companies that might be able to earn a sufficient profit and still compete with other energy sources.

The bulk of the interest in geothermal development in Colorado has come from potential users. Most share one constraint - the lack of funds. Communities have bonding 1 imits and political constraints upon obtaining loans. More importantly, their needs are large relative to their revenues, with needs other than heating systems taking precedence. Furthermore, since Federal grants are available for most community facilities, they have come to be expected. Small 
business and prospective individual users lack the means to generate sufficient front-end capital. The preparation of a Federal loan guarantee application is considered to be too costly and complex for many prospective developers. The ability of existing and planned development to stimulate subsequent development is not certain. It could, in extreme cases, have the opposite effect. Communities, hoping for federal funding such as Pagosa Springs will receive, may fail to take advantage of private or other opportunities for development. or, if a development has undesirable side-effects, those side-effects may negatively effect the image of geothermal energy. In the usual situation, the successful demonstration of geothermal energy will probably enhance geothermal development over the long term. For the short term, however, prospective users and small business will continue to be constrained from development simply because they 1 ack adequate funding.

Interest is keen from both private and public prospective developers in obtaining grant funds for development of geothermal energy for direct use. Numerous requests are heard too, for technical assistance including information about the permits required, the resources, the techniques for development and the feasibility of development. Lacking extensive information, prospective developers cannot or will not risk large sums if they do have such capital available. 
A number of actions are recommended to eliminate or alleviate geothermal development barriers or to provide incentives to geothermal development. For the most part, recommendations for stimulation measures are tentative. Barriers have been observed and reported. However, the prediction of the impacts of certain measures is largely a matter of judgement, requiring further study and documentation. The recommended actions are as follows:

1) Issuance of leases to encourage further exploration of potential high temperature sites. Increased effort at the Federal level to resolve conflicts among federal agency mandates and/or policies is recommended to at least clarify and consolidate the Federal policy.

If leases are issued, high temperature geothermal resources seem 1 ikely to be developed by established energy companies. Development of lower temperature resources for direct use will be much more difficult to attain.

2) Because exploration is a high-cost item, a risky one, and a necessary prerequisite for any development, its stimulation seems particularly critical to the acceleration process. Technical assistance in the form of geological evaluations, or reservoir insurance or cost sharing for shallow wells, and/or increased state-coupled programs could show that indeed the energy is available for use. It seems essential, however, that such efforts be concentrated in areas that have high market potential.

3) Questions about the use of geothermal resources, especially where mineralization is considered to be a problem, also limit development impetus. Increased levels of preliminary engineering assistance could alleviate that constraint. And, the lack of information about the feasibility of development sometimes precludes non-Federal investments. Increased technical assistance for such preliminary studies could help encourage subsequent private or other non-DOE or non-federal investment.

4) The failure to include geothermal energy among components of national plans aimed at the reduction of reliance upon imported oil restricts the legitimacy with which geothermal energy is viewed. It is probably itself a constraint by 
reinforcing the widespread belief that development of the energy is not economically or technically feasible. The simple mention of the work "geothermal" by high-level officials could stimulate interest and subsequent development.

5) Even greater commitment by federal decision makers to significantly expand funding of geothermal programs could assure the widespread development of the energy resources. Developing that commitment can be assisted by State team's education of their congressional delegations. Grass roots support could help as well but is itself limited by the lack of visibility of the resource potential - a chicken and egg situation.

6) The final but most restrictive hindrance to geothermal energy development is the lack of funds. Coupled with the lack of information about the resources and the engineering requirements, the lack of visibility of the energy potential, and the general lack of commitment to this energy source, the lack of readily available investment or development funds assures inertia. Were grant funds to be widely available not only for demonstration projects but for develoment of known uses as well, there is little doubt that geothermal energy development would be accelerated. By thus enhancing the acceptability and visibility of the energy resource, future development could be accomplished without federal funding. Given the current scarcity of private or federal funds for either exploration or development, geothermal energy will continue to lie dormant while other resources with greater development incentives will capture the attention of energy developers. 
C.R.S. 24-65-104

25-7-108

25-8-501-508

29-20-104

30-28-111-209

$31-32-201$

$34-70-101$

34-70-104

$36-1-112$

$37-90-137$

$37-92-$

40-3-102

H.B. 1495

1540

S.B. 316

321

324

P.L. $93-410$

Allen, Cap, Timberline Academy, pers. comm.

Andrews, Lynn, Whiteman School, pers. comm.

Barrett and Pear1, An Appraisal of Colorado's Geothermal Resources, Bull. 39, Colorado Geological Survey, 1978.

Bernal, John, Whetstone, Inc., pers. comm.

Bureau of Land Management, U.S. Department of Interior, Denver, Co., unpublished data, 1978, 1979.

Butler, David, Chevron Resources, San Francisco, Ca., pers. comm.

Coe, Barbara A., Geothermal Development in Colorado: Processes, Promises and Problems, Inf. Series 9, Colorado Geological Survey, 1978.

Colorado Board of Land Commissioners, Leasing Records, 1978, 1979.

Colorado Division of Planning, From Bonanza to Last Chance, Denver, Co., 1978 .

Cuniff, Roy, and others, New Mexico Energy Institute, unpublished data, $1978,1979$.

Dick, Jay, Chaffee Geothermal, pers. comm.

Dolan, William, Amax Exploration, pers. comm.

Ebeling, Fred, Archuleta County Planning office, pers. comm. 


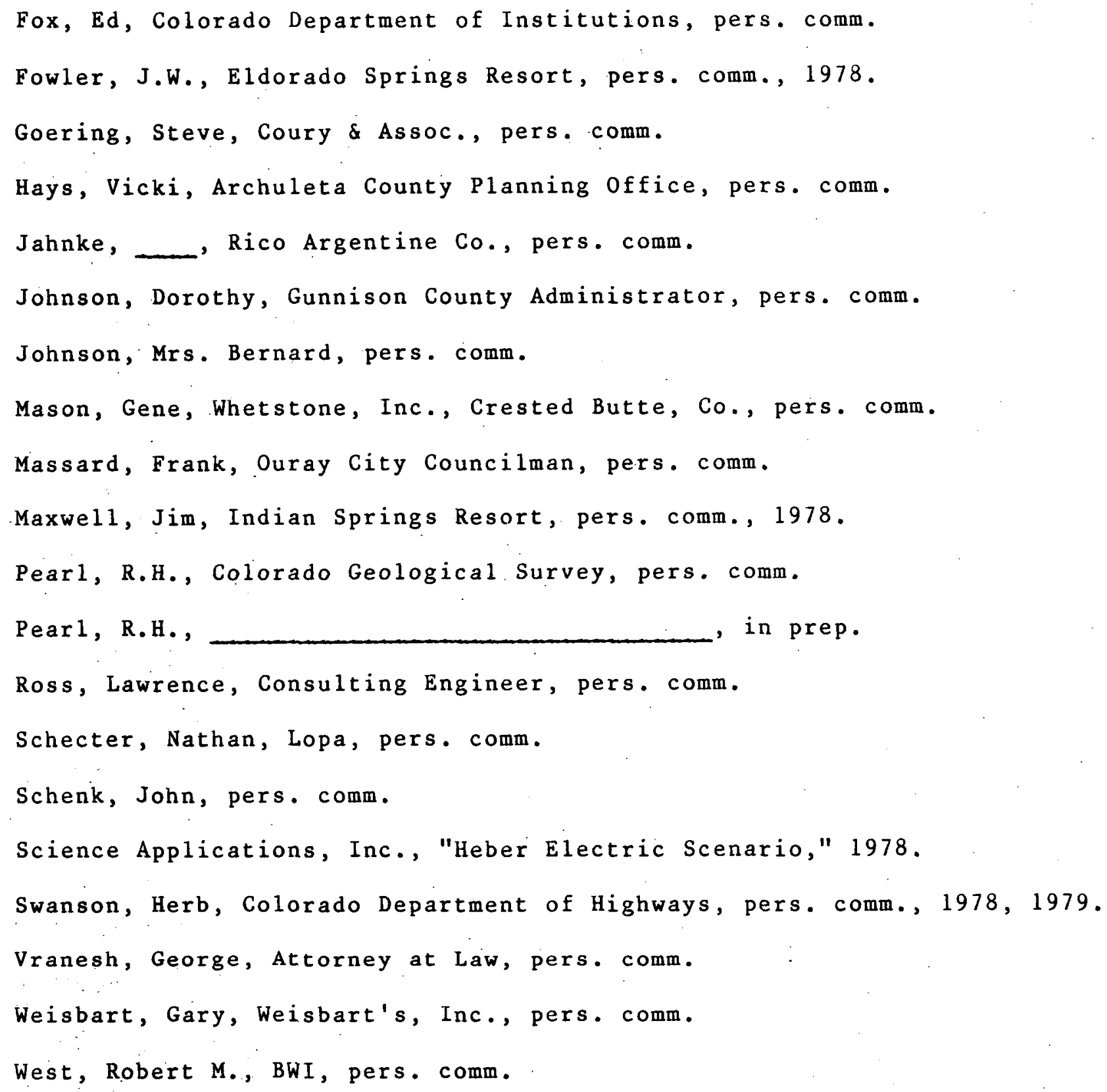


DISTRIBUTION RECORD FOR DOE/ID/12018-1

Internal Distribution

$$
\begin{aligned}
1 \text { - } & \text { Chicago Patent Group - DOE } \\
& 9800 \text { South Cass } \\
& \text { Argonne, IL } 60439 \\
1 \text { - R. L. Blackledge } & \text { Idaho Operations Office - DOE } \\
& \text { Idaho Falls, ID } 83401 \\
1 \text { - } & \text { H. P. Pearson } \\
& \text { Information Processing - EG\&G } \\
6 \text { - } & \text { INEL Technical Library } \\
100 \text { - } & \text { Special Internal }
\end{aligned}
$$

External Distribution

$$
\begin{aligned}
& \text { 2- Technical Information Center - DOE } \\
& \text { Box } 62 \\
& \text { Oak Ridge, TN } 37830
\end{aligned}
$$

Total Copies Printed: 111 